TRANSACTIONS OF THE

AMERICAN MATHEMATICAL SOCIETY

Volume 363, Number 2, February 2011, Pages 699-761

S 0002-9947(2010)05050-0

Article electronically published on September 24, 2010

\title{
SORTABLE ELEMENTS IN INFINITE COXETER GROUPS
}

\author{
NATHAN READING AND DAVID E. SPEYER
}

\begin{abstract}
In a series of previous papers, we studied sortable elements in finite Coxeter groups, and the related Cambrian fans. We applied sortable elements and Cambrian fans to the study of cluster algebras of finite type and the noncrossing partitions associated to Artin groups of finite type. In this paper, as the first step towards expanding these applications beyond finite type, we study sortable elements in a general Coxeter group $W$. We supply uniform arguments which transform all previous finite-type proofs into uniform proofs (rather than type by type proofs), generalize many of the finite-type results and prove new and more refined results. The key tools in our proofs include a skew-symmetric form related to (a generalization of) the Euler form of quiver theory and the projection $\pi_{\downarrow}^{c}$ mapping each element of $W$ to the unique maximal $c$-sortable element below it in the weak order. The fibers of $\pi_{\downarrow}^{c}$ essentially define the $c$-Cambrian fan. The most fundamental results are, first, a precise statement of how sortable elements transform under (BGP) reflection functors and second, a precise description of the fibers of $\pi_{\downarrow}^{c}$. These fundamental results and others lead to further results on the lattice theory and geometry of Cambrian (semi)lattices and Cambrian fans.
\end{abstract}

\section{Contents}

1. Introduction

Part I: Fundamental results about sortable elements

2. Coxeter groups and sortable elements 704

3. The Euler form $E_{c}$ and the form $\omega_{c} \quad 716$

4. Orientation and alignment 720

5. Forced and unforced skips

6. The projection $\pi_{\downarrow}^{c} \quad 730$

Part II: The Cambrian semilattice and fan

7. The Cambrian semilattice 736

8. Canonical join representations 739

9. The Cambrian fan 744

10. Rank three examples 749

Acknowledgments $\quad 759$

References $\quad 759$

Received by the editors February 25, 2009 and, in revised form, March 18, 2009.

2010 Mathematics Subject Classification. Primary 20 F55.

The second author was supported by a research fellowship from the Clay Mathematics Institute. 


\section{INTRODUCTION}

This paper is the continuation of a series of papers [31, 32, 33, 34] devoted to the study of sortable elements and Cambrian lattices in a Coxeter group $W$. The primary motivations behind this work are the connections to cluster algebras (see [18] and [34) and to the study of Artin groups via noncrossing partitions (see e.g. 4, 8]). In the first three of these papers, specific connections were conjectured between sortable elements/Cambrian lattices and generalized associahedra (see [16]) and thus between sortable elements and cluster algebras of finite type (see [17]). Two bijections were established: one from sortable elements to vertices of the (simple) generalized associahedron and another from sortable elements to noncrossing partitions. In 34 the former bijection was shown to induce a combinatorial isomorphism between the Cambrian fan and the normal fan of the generalized associahedron, leading to further results connecting the combinatorics of cluster algebras to the geometry of Cambrian fans. This last paper proved all of the remaining earlier conjectures concerning relations between sortable elements and finite type cluster algebras.

The results of the earlier papers were confined to the very special case where the Coxeter group $W$ is finite. In this paper, we extend the theory to the case of arbitrary $W$. Although the infinite case is a vastly larger arena where significantly fewer tools are available, we not only generalize the basic results on sortable elements, but also greatly improve them. Among the improvements is an explicit pattern avoidance characterization of $c$-sortable elements. In 32, the first author gave a pattern avoidance condition based on a family of "c-orientations," which were constructed explicitly type by type. We now construct the orientations uniformly, via the restriction of a skew symmetric bilinear form $\omega_{c}$ to parabolic subgroups. Another improvement is in our understanding of the Cambrian congruence, a certain equivalence relation on the Coxeter group $W$. In [33, the Cambrian congruence was constructed in two ways: as the lattice congruence generated by a small explicit set of equivalences, and as the fibers of a recursively defined map $\pi_{\downarrow}^{c}$. In neither case, given a representative element of a Cambrian congruence class, was it clear how to describe all elements in that equivalence class. In Theorem 6.3, we give a description of any Cambrian congruence class by a finite list of immediately checkable conditions.

The description of the equivalence classes is intimately related to a third significant improvement to the theory: Using the combinatorics of a special "sorting word" for each sortable element, we construct a simplicial cone for each sortable element. In finite type, the collection of all such cones is the Cambrian fan, which was shown in 34 to be isomorphic to the cluster complex of the corresponding cluster algebra of finite type. We do not yet prove that this collection of cones is a fan in infinite case. However, we do prove that intersecting each cone with the Tits cone produces a collection of cones whose intersections are well-behaved. The combinatorics of sorting words gives very precise combinatorial control over the Cambrian cones. This precise control will be used in a future paper to generalize and extend the results of 34 connecting the Cambrian fan to cluster algebras. (See Section 1.2 , )

Although this paper improves the general theory of sortable elements, it by no means supersedes the previous papers. The main point of each paper, and the bulk of the arguments, are not replaced by the arguments of the present paper. 
(The main points of [31, 32, 33, 34 are respectively: a lattice-theoretic and fiberpolytopal approach to generalized associahedra; using sortable elements to make a combinatorial connection between generalized associahedra and noncrossing partitions; using sortable elements to explicitly construct Cambrian lattices; and making the connection between sortable elements/Cambrian fans and the combinatorics of cluster algebras of finite type.) The current paper does greatly improve the previous papers by providing uniform proofs for a small number of lemmas in 32 which were previously proved using type by type checks. These lemmas occupy very little space in [32, but all of the main results of [32, 33, 34] ultimately depend on the lemmas. With the addition of this paper, these lemmas, and thus all of the results of the previous papers, have uniform proofs. (See Section 1.3 for details.)

1.1. Summary of results. We now summarize the results of the paper. Most of the notation and definitions surrounding Coxeter groups are put off until Section 2

An element $c \in W$ is called a Coxeter element if it is of the form $s_{1} s_{2} \cdots s_{n}$ for some ordering of the set $S:=\left\{s_{1}, s_{2}, \ldots, s_{n}\right\}$ of simple reflections. For any word $z$ in the alphabet $S$, the support $\operatorname{supp}(z) \subseteq S$ of $z$ is the set of letters occurring in $z$. An element $w \in W$ is called $c$-sortable if $w$ has a reduced word which is the concatenation $z_{1} z_{2} \cdots z_{k}$ of words $z_{i}$ subject to the following conditions:

(1) Each $z_{i}$ is a subword of $s_{1} \cdots s_{n}$.

(2) The supports of the $z_{i}^{\prime}$ 's satisfy $\operatorname{supp}\left(z_{1}\right) \supseteq \operatorname{supp}\left(z_{2}\right) \supseteq \cdots \supseteq \operatorname{supp}\left(z_{k}\right)$.

This word is called a $c$-sorting word for $w$. The definition of $c$-sortability depends on the choice of $c$ but not on the choice of reduced word for $c$. As described in Proposition 2.29, $c$-sortability can also be characterized recursively by induction on the length of $w$ and on the rank of $W$.

Before summarizing our results in the order they appear, we wish to emphasize two fundamental results on sortable elements. These results appear late in the paper and use most of the tools developed over the course of the paper. Yet if these two results could be proven first, most of the other results on sortable elements would follow.

The first result that we wish to emphasize is the following: For any $w \in W$ there exists a unique maximal $c$-sortable element below $w$. The map $\pi_{\downarrow}^{c}$ mapping $w$ to this maximal element is of fundamental importance. In particular, the fibers of $\pi_{\downarrow}^{c}$ essentially define the $c$-Cambrian fan. More precisely, the maximal cones in the $c$-Cambrian fan, intersected with the Tits cone, are the fibers of $\pi_{\downarrow}^{c}$. Proving the existence of a unique maximal $c$-sortable element below each $w$ requires all the tools developed in the first part of the paper. We therefore begin our treatment of $\pi_{\downarrow}^{c}$ with a recursive definition of $\pi_{\downarrow}^{c}(w)$. We only later establish, as Corollary 6.2. that $\pi_{\downarrow}^{c}(w)$ can be described as it is in this paragraph.

The second result describes what happens when we change Coxeter elements. We say $s \in S$ is initial in a Coxeter element $c$ if $c$ has a reduced word starting with $s$. In this case $s c s$ is another Coxeter element, obtained by removing $s$ from the beginning of the word and placing it at the end. This operation on $c$ corresponds to applying a (BGP) reflection functor in the sense of quiver theory. See [2, Section VIII.5] or [3]. If $s$ is initial in $c$, then the map

$$
v \mapsto\left\{\begin{array}{cc}
s v & \text { if } v \geq s, \\
s \vee v & \text { if } v \nsupseteq s
\end{array}\right.
$$


is a bijection from the set $\{c$-sortable elements $v$ such that $s \vee v$ exists $\}$ to the set of all scs-sortable elements. (Cf. [33, Remark 3.8] and the discussion preceding [34, Lemma 7.5].) This result, with an explicit inverse map, appears as Theorem [7.4]

In finite type this bijection implies that the number of $c$-sortable elements is independent of $c$, using the fact that the weak order is a lattice in finite type and the following (essentially graph-theoretic) result: If $c$ and $c^{\prime}$ are Coxeter elements in a finite Coxeter group $W$, then $c$ and $c^{\prime}$ are related by a sequence of moves which remove an initial letter and append it at the end.

We now describe in order the main results of the paper. In Section 3 we introduce a technical device known as the Euler form. This is a certain nonsymmetric bilinear form, denoted $E_{c}$, which is inspired by constructions in quiver theory. The symmetrization of $E_{c}$ is the ordinary inner product; the antisymmetrization $\omega_{c}$ of $E_{c}$ is a skew-symmetric bilinear form. The main result of Section 3 is Proposition 3.11 . which states that $w \in W$ is $c$-sortable if and only if $w$ has a reduced word whose associated sequence of reflections is compatible with $\omega_{c}$, in a sense we describe in that section. In Section 4 we characterize $c$-sortability of $w$ directly in terms of the set of inversions of $w$, without requiring a particular reduced word. This description identifies the property of $c$-sortability as a condition of pattern avoidance in the sense of Billey and Braden [5] and extends the first author's results in [32, Sections 3-4].

We next define, in Section 5 , a collection $C_{c}(v)$ of $n$ roots for each $c$-sortable element $v$. (Here $n$ is the rank of $W$.) This set of roots defines a dual cone $\mathrm{Cone}_{c}(v)$, the $c$-Cambrian cone associated to $v$. Using Proposition 3.11 and the Euler form, we then characterize $C_{c}(v)$ in terms of the combinatorics of $c$-sorting words. In Section 6 we give the recursive definition of $\pi_{\downarrow}^{c}$ and use a complicated inductive argument to prove two major theorems (Theorems 6.1 and 6.3). The first of these states that $\pi_{\downarrow}^{c}$ is order preserving and the second states that each fiber $\left(\pi_{\downarrow}^{c}\right)^{-1}(v)$ is the intersection of the Tits cone with $\operatorname{Cone}_{c}(v)$. Given the combinatorial characterization of $C_{c}(v)$, the latter assertion amounts to an extremely explicit description of the fibers of $\pi_{\downarrow}^{c}$.

At this point, the paper changes tone. Until now, the focus has been on technical results about sortable elements, proved via very detailed lemmas. The attention now shifts to the development of the lattice theory and polyhedral geometry of sortable elements. Each of our two primary objects, the $c$-Cambrian semilattice and the $c$-Cambrian fan, can be viewed as a way of organizing the set of fibers of $\pi_{\downarrow}^{c}$. The proofs are often short consequences of general principles and technical lemmas proved previously. We feel that the sortable elements are the girders of our theory, underlying all of our results, while the Cambrian semilattice and Cambrian fan are the doorways through which connections to other mathematical fields will be made.

The c-Cambrian semilattice is defined as the weak order restricted to $c$ sortable elements. In Section 7 we show that $c$-sortable elements are a sub-semilatice of the weak order (Theorem 7.1). Furthermore, Theorem 7.3 states that the map $\pi_{\downarrow}^{c}$ respects the meet operation and the (partially defined) join operation. In particular the $c$-Cambrian semilattice is isomorphic to the partial order induced by the weak order on the fibers of $\pi_{\downarrow}^{c}$. Theorem 7.1 leads to the proof of Theorem 7.4 which establishes the bijection, described above, from (almost all of) the $c$-sortable 
elements to the $s c s$-sortable elements. In Section 8 we discuss canonical join representations of sortable elements. This discussion leads to a bijection between $c$-sortable join-irreducible elements and reflections in $W$ which can appear as inversions of $c$-sortable elements. This bijection leads to a surprising lattice-theoretic interpretation of noncrossing partitions. As we explain in Section 8, c-noncrossing partitions encode the canonical join representations of $c$-sortable elements.

In Section 9 we show that the Cambrian cones $\operatorname{Cone}_{c}(v)$ are the maximal cones of a fan inside the Tits cone, meaning that the intersections of Cambrian cones are well-behaved inside the Tits cone. We call this fan the $c$-Cambrian fan. We conjecture a stronger statement that the Cambrian cones are the maximal cones of a fan, i.e. that they intersect nicely even outside of the Tits cone. Section 10 consists of pictorial examples illustrating many of the results of the paper; we encourage the reader to consult Section 10 frequently while reading the prior sections.

1.2. Future directions. One of the primary motivations for studying sortable elements and Cambrian lattices in this and in earlier papers is the goal of using sortable elements to study the structure of cluster algebras. We now briefly outline work in progress which will apply the results of this paper to cluster algebras. We assume familiarity with cluster algebras for this discussion; to the reader looking for background we suggest the early sections of [18].

The initial data defining a cluster algebra (of geometric type) is an integer $m \times n$ matrix $\tilde{B}$ (with $m \geq n$ ) satisfying conditions given in [18, Definition 2.12]. The most important part of $\tilde{B}$ is its top square submatrix $B$; the remaining entries of $\tilde{B}$ should be thought of as a choice of coefficients. Specifying $B$ is equivalent to choosing a symmetrizable integer generalized Cartan matrix $A$ and choosing an orientation of the Coxeter graph $\Gamma$ of the Coxeter group $W$ determined by $A$. If the orientation of $\Gamma$ is acyclic, then it defines a Coxeter element $c$ of $W$. Different choices of $\tilde{B}$ can give isomorphic cluster algebras; the main result of [17] is that a cluster algebra is of finite type if and only if it can be specified by a matrix $\tilde{B}$ such that the associated Coxeter group $W$ is finite. In this case the orientation of $\Gamma$ is necessarily acyclic and thus is equivalent to a choice of Coxeter element for $c$.

A cluster algebra (of geometric type) is generated by a collection of multivariate rational functions, known as cluster variables, which are grouped into overlapping $n$-element sets called clusters. Given $\tilde{B}$ and the choice of an initial cluster, the remaining clusters are determined by local moves called mutations. The mutation moves also assign to each cluster its own $m \times n$ matrix. Each cluster variable is assigned a vector known as the $g$-vector [18. The $g$-vector fan is a fan whose rays are the $g$-vectors. A collection of rays spans a maximal cone of the fan if and only if the corresponding collection of cluster variables is a cluster.

In 34 we proved two results concerning cluster algebras of finite type. The first result is that, for a special choice of $c$, the $c$-Cambrian fan coincides with the $g$-vector fan. Assuming [18, Conjecture 7.12], the fans coincide for all choices of $c$. In a future paper, we will use the results of this paper to remove the dependence on [18, Conjecture 7.12] and to give a generalization to infinite groups; in the finite crystallographic case, this result also appears in [36].

The second result asserts that, for each maximal cone in the $c$-Cambrian fan, the matrix of inner products of the normals to the walls of the cone is a so-called "quasiCartan" companion for the corresponding cluster. This means that the geometry of the cone in the $c$-Cambrian fan determines the absolute values of the entries in 
the $B$-matrix of the cluster. In the future paper, we will also use the results of this paper to determine the signs of the entries of $B$ and to determine, in the case of a cluster algebra with principal coefficients (see [18, Definition 3.1]), the entries of $\tilde{B}$ which do not lie in the square submatrix $B$.

The broader goal of this line of research is to study cluster algebras without restriction to finite type. The first step towards that broader goal is to extend the results of [34] beyond finite type. Specifically:

Problem 1.1. For an arbitrary cluster algebra of geometric type, construct the $g$-vector fan and the $B$-matrices, in the context of sortable elements/Cambrian fans.

In moving beyond finite type, there are two independent obstacles to overcome. First, while every cone of the Cambrian fan meets the Tits cone, examples show that there are many cones in the $g$-vector complex which do not meet the Tits cone at all. A future paper will overcome this obstacle in part: We will show how to solve Problem 1.1 in an intermediate case between finite type and the completely general case: the case where $A$ is of affine type. The second obstacle to moving beyond finite type is that our methods, thus far, can only address acyclic orientations of the Coxeter diagram. An extension of the definition of sortable elements and Cambrian fans to the non-acyclic case will appear in a future paper.

1.3. Uniform proofs of previous results. This section is included for the benefit of those citing specific results from previous papers in the series [32, 33, 34]. All of the nontrivial general results of these three papers depend on one or more of the following five results in [32] which are proved with a type-by-type check: 32, Propositions 3.1 and 3.2] and [32, Lemmas 4.6, 6.6 and 6.7]. The proofs in [33, 34] are non-uniform only to the extent to which they quote results from [32.

All five of these results are given uniform proofs in this paper, so that in particular all results of [32, 33, 34] now have uniform proofs. Specifically, in light of Lemmas 3.8 and 3.9, the forms $\omega_{c}$ (as $c$ varies) provide the family of "orientations" of $W$ whose existence is asserted in [32, Proposition 3.1], and thus Lemma 3.7 implies [32, Proposition 3.2]. The function of [32, Lemma 4.6] is to provide one case in the inductive proof of 32 , Theorem 4.1], which is generalized by Theorem 4.3 in

the present paper. The proof of Theorem 4.3 has the same inductive structure as the proof of [32, Theorem 4.1] and, indeed the generalization of [32, Lemma 4.6] is proved in the course of the proof of Theorem 4.3. Finally, [32, Lemmas 6.6 and 6.7] are generalized by Propositions 5.3 and 5.4 .

We caution the reader that, while we now have a uniform proof of [33, Theorem 1.4], the formulation of this result requires $W$ to be a lattice, and hence finite. We have not found a correct generalization of [33, Theorem 1.4] to infinite $W$.

\section{PART I: Fundamental RESUlts ABOUt SORTABle ELEMENTS}

\section{Coxeter groups And SORTABle Elements}

In this section we establish notation and conventions for Coxeter groups and root systems and then review the definition of sortable elements. We assume familiarity with the most basic definitions and results on Coxeter groups. (See, for example, 6. 7, 21.) All of the notation in this section is fairly standard. 
2.1. Coxeter groups. Throughout the paper, $W$ will be a Coxeter group with simple generators $S$. The identity element will be denoted by $e$. The labels on the Coxeter diagram for $W$ will be written $m\left(s, s^{\prime}\right)$. We permit $m\left(s, s^{\prime}\right)=\infty$. The rank of $W$ is the cardinality of $S$, which will be assumed to be finite and will be called $n$. The length of $w \in W$, written $\ell(w)$, is the number of letters in a reduced (i.e. shortest possible) word for $w$ in the alphabet $S$. The support of $w$ is the set of generators occurring in a reduced word for $w$. This is independent of the choice of reduced word.

A reflection in $W$ is an element of $W$ which is conjugate to some element of $S$. The symbol $T$ will stand for the set of reflections in $W$. An inversion of $w$ is a reflection $t$ such that $\ell(t w)<\ell(w)$. The set of inversions of $w$, written $\operatorname{inv}(w)$, uniquely determines $w$. Given any reduced word $a_{1} \cdots a_{k}$ for $w$, the inversion set of $w$ consists of $k$ distinct reflections. These are of the form $t_{i}=a_{1} \cdots a_{i} \cdots a_{1}$ for $1 \leq i \leq k$. The sequence $t_{1}, \ldots, t_{k}$ is the reflection sequence for the reduced word $a_{1} \cdots a_{k}$. Since $w=t_{k} t_{k-1} \cdots t_{1}$, we have the following lemma.

Lemma 2.1. Let $u, w \in W$ and suppose $u$ commutes with every inversion of $w$. Then u commutes with $w$.

A cover reflection of $w \in W$ is an inversion $t$ of $w$ such that $t w=w s$ for some $s \in S$. The name "cover reflection" refers to the fact that $w$ covers $t w$ in the weak order (defined below). The set of cover reflections of $w$ is written $\operatorname{cov}(w)$. If $t$ is a cover reflection of $w$, then $\operatorname{inv}(t w)=\operatorname{inv}(w) \backslash\{t\}$.

Suppose $s \in S$ and $w \in W$ such that $\ell(s w)<\ell(w)$. Then the inversion sets of $w$ and $s w$ are related as follows: $\operatorname{inv}(w)=\{s\} \cup\{s t s: t \in \operatorname{inv}(s w)\}$. If $s$ is a cover reflection of $w$, then $\operatorname{inv}(s w)=\operatorname{inv}(w) \backslash\{s\}$, so that $\operatorname{inv}(s w)=\{s t s: t \in \operatorname{inv}(s w)\}$.

2.2. Root systems. We now fix a reflection representation for $W$ in the standard way by choosing a root system. A generalized Cartan matrix for a Coxeter group $W$ is a real matrix $A$ with rows and columns indexed by $S$ such that:

(i) $a_{s s}=2$ for every $s \in S$;

(ii) $\quad a_{s s^{\prime}} \leq 0$ with $a_{s s^{\prime}} a_{s^{\prime} s}=4 \cos ^{2}\left(\frac{\pi}{m\left(s, s^{\prime}\right)}\right)$ when $s \neq s^{\prime}$ and $m\left(s, s^{\prime}\right)<\infty$, and $a_{s s^{\prime}} a_{s^{\prime} s} \geq 4$ if $m\left(s, s^{\prime}\right)=\infty$; and

(iii) $a_{s s^{\prime}}=0$ if and only if $a_{s^{\prime} s}=0$.

Most references, such as [23, Chapter 1], require that $A$ have integer entries. We permit $A$ to have real entries and say that $A$ is crystallographic if it has integer entries.

Let $V$ be a real vector space of dimension $n$ with a basis $\alpha_{s}$ labeled by the simple reflections and let $V^{*}$ be the dual vector space; the set $\Pi:=\left\{\alpha_{s}: s \in S\right\}$ is called the set of simple roots. The canonical pairing between $x^{*} \in V^{*}$ and $y \in V$ will be written $\left\langle x^{*}, y\right\rangle$. The vector space $V$ carries a reflection representation of $W$ such that $s \in S$ acts on the basis element $\alpha_{s^{\prime}}$ by $s\left(\alpha_{s^{\prime}}\right)=\alpha_{s^{\prime}}-a_{s s^{\prime}} \alpha_{s}$. When necessary for clarity, we will denote $V$ and $V^{*}$ by $V(W)$ and $V^{*}(W)$. The action of $w \in W$ on a vector $x \in V$ will be denoted $w x$, and the same notation will be used for the dual action on $V^{*}$. (This is the natural action on the dual, which is defined whether or not we have any way of identifying $V$ and $V^{*}$.)

We will restrict our attention to symmetrizable generalized Cartan matrices. We say that $A$ is symmetrizable if there exists a positive real-valued function $\delta$ on $S$ such that $\delta(s) a_{s s^{\prime}}=\delta\left(s^{\prime}\right) a_{s^{\prime} s}$ and, if $s$ and $s^{\prime}$ are conjugate, then $\delta(s)=\delta\left(s^{\prime}\right)$. 
(This second condition is nonstandard; we will discuss it further in Section 2.3.) We set $\alpha_{s}^{\vee}=\delta(s)^{-1} \alpha_{s}$; the $\alpha^{\vee}$ are called the simple co-roots 1 Define a bilinear form $K$ on $V$ by $K\left(\alpha_{s}^{\vee}, \alpha_{s^{\prime}}\right)=a_{s s^{\prime}}$. Note that $K\left(\alpha_{s}, \alpha_{s^{\prime}}\right)=\delta(s) K\left(\alpha_{s}^{\vee}, \alpha_{s^{\prime}}\right)=\delta(s) a_{s s^{\prime}}=$ $\delta\left(s^{\prime}\right) a_{s^{\prime} s}=K\left(\alpha_{s^{\prime}}, \alpha_{s}\right)$, so $K$ is symmetric. One easily verifies that $W$ acts on the basis of simple co-roots by $s\left(\alpha_{s^{\prime}}^{\vee}\right)=\alpha_{s^{\prime}}^{\vee}-a_{s^{\prime} s} \alpha_{s}^{\vee}$. The action of $W$ preserves $K$.

Example 2.2. Consider the group $B_{2}$. This is a Coxeter group of rank two, with generators $p$ and $q$, and $m(p, q)=4$. We take our Cartan matrix to be $A=\left(\begin{array}{cc}2 & -2 \\ -1 & 2\end{array}\right)$. Therefore, $A$ is crystallographic, and is symmetrizable with $\delta(p)=1 / 2$ and $\delta(q)=2$. On the left hand side of Figure 1 we depict the roots and co-roots of $B_{2}$.
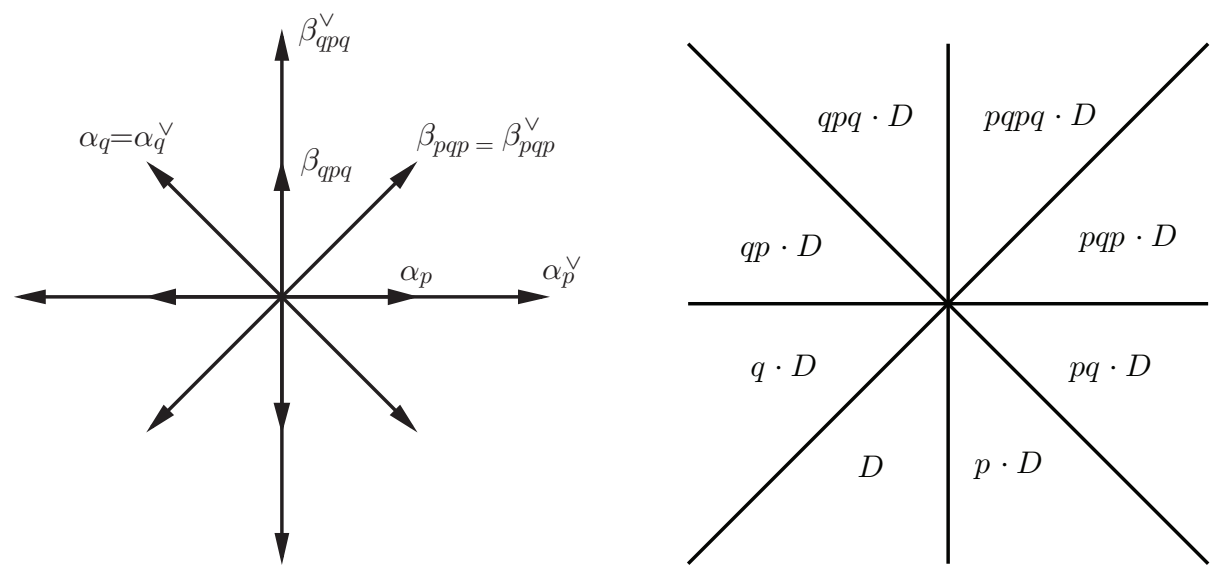

Figure 1. The $B_{2}$ root system and hyperplane arrangement.

We emphasize that we use symmetrizability only to prove combinatorial results which depend on the group $W$ but which are intrinsically independent of the choice of $A$. In Section 9 we describe the $c$-Cambrian fan, whose geometry (but not whose combinatorics) depends on the choice of Cartan matrix $A$. The results on the Cambrian fan are valid for any choice of $A$, since they rely only on the combinatorial results of the previous sections. For our present purposes, we could restrict further to the symmetric case where $\delta$ is identically 1 . (Note, for any $W$, we can make a symmetric choice of $A$.) However, we would then have to introduce a new set of conventions when relating the $c$-Cambrian fan to cluster algebras, so we work with the symmetrizable case from the beginning.

The set of vectors of the form $w \alpha_{s}$, for $s \in S$ and $w \in W$, is the root system $\Phi$, and its elements are called roots 2 The positive roots $\Phi_{+}$are the roots in $\Phi$ which are in the positive linear span of $\Pi$. Since the simple roots are linearly independent, each positive root has a unique expression as a positive combination of simple roots. The negative roots are $\Phi_{-}=-\Phi_{+}$and $\Phi=\Phi_{+} \cup \Phi_{-}$. There is a bijection $t \mapsto \beta_{t}$ between

\footnotetext{
${ }^{1}$ The standard way of proceeding, described in [23. Chapters 1-3], is more sophisticated than this: One first constructs a pair of dual vector spaces $\mathfrak{h}$ and $\mathfrak{h}^{*}$, containing the roots and co-roots respectively, and later builds an isomorphism $\nu$ between $\mathfrak{h}$ and $\mathfrak{h}^{*}$. We start off by constructing the roots and co-roots in the same space to begin with, and only look at the subspace $V$ of $\mathfrak{h}$ which is spanned by the roots.

${ }^{2}$ In some contexts, these are called real roots.
} 
the reflections $T$ in $W$ and the positive roots 3 We define $\beta_{t}^{\vee}=\left(2 / K\left(\beta_{t}, \beta_{t}\right)\right) \beta_{t}$. If $t=w s w^{-1}$, then $\beta_{t}^{\vee}=\delta(s)^{-1} \beta_{t}$. The action of $t$ on $V$ is by the relation $t \cdot x=x-K\left(\beta_{t}^{\vee}, x\right) \beta_{t}=x-K\left(x, \beta_{t}\right) \beta_{t}^{\vee}$. In the action of $W$ on $V^{*}$, the element $t$ is represented by a reflection fixing the hyperplane $H_{t}=\left\{x^{*} \in V^{*}:\left\langle x^{*}, \beta_{t}\right\rangle=0\right\}$. Under this bijection, $\alpha_{s}=\beta_{s}$ and $\alpha_{s}^{\vee}=\beta_{s}^{\vee}$ for each $s \in S$.

For $w \in W$ and $t \in T$, the reflection $t$ is an inversion of $w$ if and only if $w^{-1} \beta_{t}$ is a negative root. The action of a simple generator $s \in S$ on a positive root is $s \beta_{t}=\beta_{s t s}$ unless $t=s$, in which case we have $s \alpha_{s}=-\alpha_{s}$.

We now review the polyhedral geometry of the representation of $W$ in $V^{*}$. A polyhedral cone in $V^{*}$ is the intersection of finitely many closed halfspaces whose boundary hyperplane contains the origin. A face of a polyhedral cone $C$ is a set of the form $\{x \in C: \lambda(x)=0\}$, where $\lambda$ is a real-valued linear function which is nonnegative on $C$. A polyhedral cone is pointed if its minimal face is the origin. A simplicial cone is a polyhedral cone of full dimension in $V^{*}$ such that the normal vectors to the defining halfspaces are a basis for $V$. A set $\mathcal{F}$ of polyhedral cones in a real vector space is a $\boldsymbol{f a n}$ if

(i) for every $F \in \mathcal{F}$, the faces of $F$ are also in $\mathcal{F}$ and

(ii) for any subset $\mathcal{X} \subset \mathcal{F}$, the intersection $\bigcap_{G \in \mathcal{X}} G$ is a face of $F$ for each $F \in \mathcal{X}$.

The dominant chamber in $V^{*}$ is the set

$$
D=\bigcap_{s \in S}\left\{v^{*} \in V^{*}:\left\langle v^{*}, \alpha_{s}\right\rangle \geq 0\right\} .
$$

The dominant chamber is an $n$-dimensional simplicial cone. The set $\mathcal{F}(W)$ of all cones $w D$ and their faces is a fan in $V^{*}$ which we call the Coxeter fan. The union of the cones of $\mathcal{F}(W)$ is a convex subset of $V^{*}$ known as the Tits cone and denoted $\operatorname{Tits}(W)$. (Both $\mathcal{F}(W)$ and $\operatorname{Tits}(W)$ depend on the choice of reflection representation of $W$.)

Each cone $w D$ is a region in the hyperplane arrangement $\mathcal{A}=\left\{H_{t}: t \in T\right\}$, meaning that $w D$ is a full-dimensional connected component of $V \backslash\left(\bigcup_{t \in T} H_{t}\right)$. The map $w \mapsto w D$ is a bijection between elements of $W$ and regions of $\mathcal{A}$ which lie in $\operatorname{Tits}(W)$. The inversion set of $w \in W$ equals $\left\{t \in T:\left\langle x^{*}, \beta_{t}\right\rangle \leq 0\right.$ for all $\left.x^{*} \in w D\right\}$, or in other words, the set of reflections $t$ such that $H_{t}$ separates $w D$ from $D$.

Example 2.3. On the right hand side of Figure 1 we illustrate the hyperplane arrangement $\mathcal{A}$ in $V^{*}$, in the case where $W$ is $B_{2}$. Since $W$ is finite in this case, the Tits cone is all of $V^{*}$. From this figure, the reader can check that, for example, the inversions of $p q$ are $p$ and $p q p$, and can similarly determine the inversions of every other element in $W$.

2.3. Conjugacy classes of reflections. In defining a symmetrizable generalized Cartan matrix, we required that $\delta(s)=\delta\left(s^{\prime}\right)$ whenever $s$ and $s^{\prime}$ are conjugate. As stated, this condition appears difficult to check. In this section, we point out that this condition is very computable and that it is automatic if $A$ is crystallographic.

Define $\Gamma^{\text {odd }}$ to be the graph whose vertex set is $S$ and where there is an edge between $s$ and $s^{\prime}$ if and only if $m\left(s, s^{\prime}\right)$ is odd. For this purpose, $\infty$ is even. The following proposition appears as Exercise 1.16.a of [6].

\footnotetext{
${ }^{3}$ This statement depends on the assumption that $\delta(s)=\delta\left(s^{\prime}\right)$ if $s$ and $s^{\prime}$ are conjugate.
} 
Proposition 2.4. Two simple reflections, $s$ and $s^{\prime}$, are conjugate if and only if they lie in the same connected component of $\Gamma^{\mathrm{odd}}$.

Corollary 2.5. Let $A$ be a crystallographic generalized Cartan matrix and let $\delta$ be a positive real-valued function on $S$ such that $\delta(s) a_{s s^{\prime}}=\delta\left(s^{\prime}\right) a_{s^{\prime} s}$ for all $s, s^{\prime} \in S$. Then $\delta(s)=\delta\left(s^{\prime}\right)$ whenever $s$ and $s^{\prime}$ are conjugate. In particular, $A$ is symmetrizable.

Proof. We may immediately reduce to the case that $s$ and $s^{\prime}$ are joined by an edge of $\Gamma^{\text {odd }}$. The requirement that $A$ be crystallographic implies that $m\left(s, s^{\prime}\right)$ is $2,3,4,6$ or $\infty$. Thus $m\left(s, s^{\prime}\right)$ is odd only when $m\left(s, s^{\prime}\right)=3$. In this case, $a_{s s^{\prime}} a_{s^{\prime} s}=1$ for nonpositive integers $a_{s s^{\prime}}$ and $a_{s^{\prime} s}$. Therefore $a_{s s^{\prime}}=a_{s^{\prime} s}=-1$. Since $\delta(s) a_{s s^{\prime}}=\delta\left(s^{\prime}\right) a_{s^{\prime} s}$ we have $\delta(s)=\delta\left(s^{\prime}\right)$, as desired.

2.4. Parabolic subgroups. A standard parabolic subgroup of $W$ is a subgroup $W_{J}$ generated by some subset $J \subseteq S$ of the simple generators. The most important case will be when $J=S \backslash\{s\}$ for some $s \in S$. We use the notation $\langle s\rangle=S \backslash\{s\}$. The standard parabolic subgroup $W_{J}$ is in particular a Coxeter group with simple generators $J$. The reflections of $W_{J}$ are exactly $T_{J}=T \cap W_{J}$.

The following lemma about standard parabolic subgroups will be useful later.

Lemma 2.6. If $w \in W_{\langle s\rangle}$, then the positive root $\beta_{w s w^{-1}}$ is in the positive linear span of $\left\{\alpha_{s}\right\} \cup\left\{\beta_{t}: t \in \operatorname{inv}(w)\right\}$.

Proof. We argue by induction on $\ell(w)$. The base case $w=e$ is trivial. If $\ell(w)>0$, then write a reduced word $r_{1} \cdots r_{\ell(w)}$ for $w$ and let $t_{i}=r_{1} \cdots r_{i} \cdots r_{1}$ for $i=$ $1, \ldots, \ell(w)$. Consider the element $w^{\prime}:=r_{2} \cdots r_{\ell(w)}$. By induction, $\beta_{w^{\prime} s\left(w^{\prime}\right)^{-1}}$ is in the positive linear span of $\left\{\alpha_{s}\right\} \cup\left\{\beta_{t}: t \in \operatorname{inv}\left(w^{\prime}\right)\right\}$. Thus $\beta_{w s w^{-1}}$ is in the positive linear span of $\left\{\beta_{r_{1} s r_{1}}\right\} \cup\left\{\beta_{t}: t \in r_{1} \operatorname{inv}\left(w^{\prime}\right) r_{1}\right\}$. But $\beta_{r_{1} s r_{1}}=\alpha_{s}-a_{s r_{1}} \alpha_{r_{1}}$ is in the positive linear span of $\alpha_{s}$ and $\alpha_{r_{1}}=\beta_{t_{1}}$. (Since $s \neq r_{1}$, we know that $a_{s r_{1}} \leq 0$.) Thus we see that $\beta_{w s w^{-1}}$ is in the positive span of $\beta_{t}$ for $t \in r_{1} \operatorname{inv}\left(w^{\prime}\right) r_{1} \cup\left\{r_{1}, s\right\}=$ $\operatorname{inv}(w) \cup\{s\}$.

Given $w \in W$ and $J \subseteq S$, the coset $W_{J} w$ contains a unique element ${ }^{J} w$ of minimal length. Equivalently, there is a unique factorization $w=w_{J} \cdot{ }^{J} w$ maximizing $\ell\left(w_{J}\right)$ subject to the requirement that $\ell\left(w_{J}\right)+\ell\left({ }^{J} w\right)=\ell(w)$. (For a dual formulation, see [6. Section 2.4].) The map $w \mapsto w_{J}$ from $W$ to $W_{J}$ is defined by the property that $\operatorname{inv}\left(w_{J}\right)=\operatorname{inv}(w) \cap W_{J}$. To see why, first write a reduced word $a_{1} \cdots a_{k}$ such that $a_{1} \cdots a_{i}$ is a reduced word for $w_{J}$, for some $i \leq k$. Then since the inversions of $w$ are of the form $t_{j}=a_{1} \cdots a_{j} \cdots a_{1}$ for $1 \leq j \leq k$, we have $\operatorname{inv}\left(w_{J}\right) \subseteq \operatorname{inv}(w) \cap W_{J}$. If there exists a reflection $t \in\left[\operatorname{inv}(w) \cap W_{J}\right] \backslash \operatorname{inv}\left(w_{J}\right)$, then there exists $j$ with $i<j \leq k$ such that $t=a_{1} \cdots a_{j} \cdots a_{1}$. Since $t w=a_{1} \cdots \widehat{a_{j}} \cdots a_{k}$ (deleting $a_{j}$ from $a_{1} \cdots a_{k}$ ), the element $u$ given by $a_{i+1} \cdots \widehat{a_{j}} \cdots a_{k}$ is $\left(w_{J}\right)^{-1} \cdot t \cdot w$. In particular, $u$ is a representative of the coset $W_{J} w$, because $t$ and $w_{J}$ are in $W_{J}$. This contradicts the fact that ${ }^{J} w$ is the minimal-length coset representative, thus proving that $\operatorname{inv}\left(w_{J}\right)=$ $\operatorname{inv}(w) \cap W_{J}$.

A parabolic subgroup of $W$ is any subgroup $W^{\prime}$ conjugate in $W$ to a standard parabolic subgroup. In particular, a parabolic subgroup $W^{\prime}=w W_{J} w^{-1}$ is generated by the reflections $\left\{w s w^{-1}: s \in J\right\}$. Thus the following theorem applies. 
Theorem 2.7. Let $W$ be a Coxeter group and let $W^{\prime}$ be a subgroup of $W$ generated by reflections. Then there is a unique subset $R \subseteq W^{\prime} \cap T$ satisfying the following two conditions:

(i) For any $t \in W^{\prime} \cap T$, the positive root $\beta_{t}$ is in the positive span of $\left\{\beta_{r}: r \in R\right\}$.

(ii) For any $r \in R$, the positive root $\beta_{r}$ is not in the positive span of the set $\left\{\beta_{t}: t \in W^{\prime} \cap T \backslash\{r\}\right\}$.

Furthermore, $\left(W^{\prime}, R\right)$ is a Coxeter system with reflections $W^{\prime} \cap T$.

We call $R$ the set of canonical generators of $W^{\prime}$. Theorem 2.7 is the main theorem of [12]; however, some details in the statement of Theorem 2.7] occur only in the proof of the main theorem of [12]. Essentially the same theorem was proved in [15] at about the same time.

A standard parabolic subgroup $W_{J}$ has canonical generators $J$. More generally, let $W^{\prime}=w W_{J} w^{-1}$ be a parabolic subgroup. Then the set $\Phi^{\prime}=w \Phi_{J} w^{-1}$ is a root system for $W^{\prime}$. Define $\Phi_{+}^{\prime}=\Phi^{\prime} \cap \Phi_{+}$and let $\Pi^{\prime}$ be the unique minimal subset of $\Phi_{+}^{\prime}$ such that any root in $\Phi_{+}^{\prime}$ is contained in the positive linear span of $\Pi^{\prime}$. Theorem 2.7 says that $\Pi^{\prime}$ is the set of positive roots associated to the canonical generators of $W^{\prime}$. We have an additional characterization of the canonical generators of parabolic subgroups. The characterization refers to the unique minimal coset representative $w^{J}$ of the coset $w W_{J}$. (The left-right dual notation ${ }^{J} w$ was used above.)

Proposition 2.8. Let $R$ be the set of canonical generators of a parabolic subgroup $W^{\prime}=w W_{J} w^{-1}$. If $w=w^{J}$, then $R=w J w^{-1}$.

Proof. Let $w=w^{J}$. We only need to show that $w J w^{-1}$ satisfies conditions (i) and (ii) of Theorem 2.7. It is enough to show that, for each reflection $u \in T \cap W_{J}$, the root $w \beta_{u}$ is positive: If so, then conditions (i) and (ii) on $w J w^{-1}$ (for the subgroup $W^{\prime}$ ) follow from conditions (i) and (ii) on $J$ (for the subgroup $W_{J}$ ).

Let $u \in T \cap W_{J}$. Now $\ell(w u)>\ell(w)$ since $w$ is the unique minimal-length coset representative of $w W_{J}$. Thus $w u w^{-1} \notin \operatorname{inv}(w)$ (since $\left.\ell\left(w u w^{-1} w\right)=\ell(w u)>\ell(w)\right)$, so $w^{-1} \beta_{w u w^{-1}}$ is a positive root. This positive root is necessarily $\beta_{w^{-1} w u w^{-1} w}=\beta_{u}$. Since $w^{-1} \beta_{w u w^{-1}}=\beta_{u}$, the root $w \beta_{u}$ is the positive root $\beta_{w u w^{-1}}$.

Let $J \subseteq S$ and let $V_{J}$ be the subspace of $V$ spanned by $\left\{\alpha_{s}: s \in J\right\}$. Then $W_{J}$ preserves $V_{J}$ and acts on $V_{J}$ by the representation $V\left(W_{J}\right)$; henceforth we will identify $V\left(W_{J}\right)$ with $V_{J}$. In particular, if $t$ is a reflection of $W_{J}$, the notation $\beta_{t}$ denotes the same vector whether we think of it as a root of $W$ or of $W_{J}$. The root system associated to $W_{J}$ is $\Phi_{J}=\Phi \cap V_{J}$. Dual to the inclusion of $V_{J}$ into $V$, there is a surjection from $V^{*}$ onto $\left(V_{J}\right)^{*}$, which we denote by $P_{J}$. The dominant chamber for $W_{J}$ in $V_{J}^{*}$ is the set

$$
D_{J}=\bigcap_{s \in J}\left\{v^{*} \in V_{J}^{*}:\left\langle v^{*}, \alpha_{s}\right\rangle \geq 0\right\}=P_{J}(D) .
$$

The projection from $W$ to $W_{J}$ satisfies $P_{J}(w D) \subseteq w_{J}\left(D_{J}\right)$, and furthermore

$$
\bigcup_{\substack{x \in W \\ x_{J}=w_{J}}} x D=\left(P_{J}\right)^{-1}\left(w_{J} D_{J}\right) \cap \operatorname{Tits}(W) .
$$

We will need a generalization of the notion of a rank two parabolic subgroup. Let $t_{1}$ and $t_{2}$ be distinct reflections of $W$. Then the generalized rank two parabolic subgroup containing $t_{1}$ and $t_{2}$ is the subgroup $W^{\prime}$ of $W$ generated by 
all reflections $t$ such that $\beta_{t}$ is in the vector space $U$ spanned by $\beta_{t_{1}}$ and $\beta_{t_{2}}$. If distinct reflections $t_{1}$ and $t_{2}$ lie in a rank two parabolic subgroup $W^{\prime}$, then $W^{\prime}$ is the generalized rank two parabolic subgroup containing $t_{1}$ and $t_{2}$. There is a geometric criterion for when a generalized rank two parabolic subgroup is actually parabolic:

Proposition 2.9. Let $p$ and $q$ be two distinct reflections of $W$. Then the generalized rank two parabolic subgroup containing $p$ and $q$ is a parabolic subgroup if and only if the convex set $H_{p} \cap H_{q} \cap \operatorname{Tits}(W)$ has dimension $n-2$.

Since $H_{p} \cap H_{q}$ is a linear space of dimension $n-2$, the condition is that a positive volume portion of this linear space meets $\operatorname{Tits}(W)$.

Proof. Suppose that $H_{p} \cap H_{q}$ meets $\operatorname{Tits}(W)$ in the required manner. The intersection $H_{p} \cap H_{q} \cap \operatorname{Tits}(W)$ is a union of faces of the Coxeter fan, so there must be some face $\gamma$ of the Coxeter fan, of dimension $n-2$, with $\gamma \subseteq H_{p} \cap H_{q}$. Let $w$ be such that $\gamma$ lies in the boundary of $w D$. Then $w^{-1}(\gamma)$ is a face of $D$ of dimension $n-2$, and must be of the form $D \cap \alpha_{r}^{\perp} \cap \alpha_{s}^{\perp}$ for some simple reflections $r$ and $s$. The reflections $w^{-1} p w$ and $w^{-1} q w$ fix $D \cap \alpha_{r}^{\perp} \cap \alpha_{s}^{\perp}$, so $w^{-1} p w$ and $w^{-1} q w$ lie in $W_{\{r, s\}}$ by [6, Lemma 4.5.1]. Thus $p$ and $q$ lie in $w W_{\{r, s\}} w^{-1}$, so the parabolic subgroup $w W_{\{r, s\}} w^{-1}$ is the generalized rank two parabolic subgroup containing $p$ and $q$.

The proof of the converse is similar, and easier.

Example 2.10. Proposition 2.9 says in particular that to find a generalized parabolic subgroup which is not parabolic, one must find hyperplanes that meet outside the Tits cone. A simple example is to take $W$ to be $\tilde{A}_{2}$, the rank three Coxeter group with $m(r, s)=m(r, t)=m(s, t)=3$. The Tits cone for $\tilde{A}_{2}$ is the union of an open half space with the singleton $\{0\}$. The reflecting planes $H_{r s r}$ and $H_{t}$ meet in a line $L$ on the boundary of the Tits cone. There are infinitely many reflections of $W$ whose reflecting planes contain $L$, so the generalized rank two parabolic subgroup containing $r s r$ and $t$ is infinite. However, all rank two parabolic subgroups of $\tilde{A}_{2}$ are finite.

Proposition 2.11. Let $W^{\prime}$ be a generalized rank two parabolic subgroup of $W$. Then $W^{\prime}$ has exactly two canonical generators, $\left\{r_{1}, r_{2}\right\}$. Furthermore, $W^{\prime} \cap T=$ $\left\{r_{1}, r_{1} r_{2} r_{1}, r_{1} r_{2} r_{1} r_{2} r_{1}, \ldots, r_{2} r_{1} r_{2} r_{1} r_{2}, r_{2} r_{1} r_{2}, r_{2}\right\}$.

Proof. Let $R$ be the set of canonical generators of $W^{\prime}$. Since $\left\{\beta_{t}: t \in W^{\prime} \cap T\right\}$ contains at least two independent vectors, $R$ has at least two elements. We claim that $R$ has exactly two elements. If $p, q$ and $r$ are distinct elements of $R$, then $\beta_{p}$, $\beta_{q}$ and $\beta_{r}$ all lie in the two-dimensional space spanned by $\beta_{t_{1}}$ and $\beta_{t_{2}}$. So there is a linear relation $a \beta_{p}+b \beta_{q}+c \beta_{r}=0$. Since $\beta_{p}, \beta_{q}$ and $\beta_{r}$ are all positive roots, $a$, $b$ and $c$ can't all have the same sign; assume without loss of generality that $a<0$ and $b, c \geq 0$. Then $\beta_{p}$ is in the positive span of $\beta_{q}$ and $\beta_{r}$, contradicting property (ii) of the set $R$.

Now, Theorem 2.7 asserts that $W^{\prime}$ is a Coxeter group with simple generators $R$ and that $W^{\prime} \cap T$ is the set of reflections of $W^{\prime}$ in this presentation. This fact implies the last assertion.

Remark 2.12. One corollary of Proposition 2.11 is that a subgroup $W^{\prime}$ of $W$ is a generalized parabolic of rank two if and only if $W^{\prime}$ is generated by two reflections and is not contained in any larger subgroup which is generated by a pair of reflections. This characterization shows that the property of being a generalized 
parabolic subgroup of rank two depends only on $W$, not on the choice of root system for $W$.

Remark 2.13. We have deliberately avoided defining generalized parabolic subgroups of rank larger than two. One could take any linear space $L \subset V$ and take the subgroup $W^{\prime}$ of $W$ generated by the reflections for the roots lying in $L$. Then Theorem 2.7 states that $\left(W^{\prime}, R\right)$ is a Coxeter system, where $R$ is the set of canonical generators of $W^{\prime}$. Surprisingly, $(W, R)$ may have rank greater than $\operatorname{dim} L$. (We thank Matthew Dyer for pointing this out to us.) For example, let $W=\tilde{A}_{3}$, the rank four Coxeter group with $m(r, s)=m(s, t)=m(t, u)=m(u, r)=3$ and $m(r, t)=m(s, u)=2$. Take $L=\left\{a \alpha_{r}+b \alpha_{s}+c \alpha_{t}+d \alpha_{u}: a+c=b+d\right\}$. One can show that the group $W^{\prime}$ generated by reflections in the roots contained in $L$ has canonical generators $\{r s r, s t s, t u t, u r u\}$. This is the Coxeter group of type $\tilde{A}_{1} \times \tilde{A}_{1}$ and rank four. The lack of control over rank forms an obstacle whenever we want to induct on rank, and we have chosen to avoid the matter altogether in this paper.

We now mention several easy lemmas about parabolic subgroups. (Cf. 32, Lemma 1.2], [32, Lemma 1.4], 32, Lemma 1.5] and [29, Lemma 6.6]. An inconsequential misstatement in [32, Lemma 1.5] is corrected in Lemma 2.16 below.) In the proofs that follow, $\Phi^{\prime}$ will denote the root system associated to $W^{\prime}$.

Lemma 2.14. Let $W^{\prime}$ be a parabolic subgroup (of any rank) or generalized rank two parabolic subgroup with canonical generators $R$. If $s \in S \cap W^{\prime}$, then $s \in R$.

Proof. The root $\alpha_{s}$ spans an extreme ray in the positive span of $\Phi_{+}$and therefore must span an extreme ray in the positive span of $\Phi_{+}^{\prime}$.

Lemma 2.15. Let $W^{\prime}$ be a parabolic subgroup (of any rank) or generalized rank two parabolic subgroup with canonical generators $R$ and let $s \in S$. If $s \notin R$, then the parabolic subgroup $s W^{\prime} s$ has canonical generators $s R s=\left\{s s^{\prime} s: s^{\prime} \in R\right\}$.

Proof. The action of $s$ permutes $\Phi_{+} \backslash\left\{\alpha_{s}\right\}$. Therefore the positive roots associated to $s W^{\prime} s$ are $s\left(\Phi_{+}^{\prime}\right)$.

Lemma 2.16. For any standard parabolic subgroup $W_{J}$ and any generalized rank two parabolic subgroup $W^{\prime}$, the intersection $W^{\prime} \cap W_{J}$ is either $\{e\}$, a pair $\{e, p\}$ where $p$ is a canonical generator of $W^{\prime}$, or all of $W^{\prime}$.

Proof. If $\Phi^{\prime} \cap \Phi_{J}=\emptyset$, then $W^{\prime} \cap W_{J}=\{e\}$. If $\Phi^{\prime} \subseteq \Phi_{J}$, then $W^{\prime} \cap W_{J}=W^{\prime}$. Otherwise, since both $\Phi^{\prime}$ and $\Phi_{J}$ can be described as the intersection of $\Phi$ with a subspace, $\Phi^{\prime} \cap \Phi_{J}$ must span a line. In this case, $W^{\prime} \cap W_{J}=\{e, t\}$ for some reflection $t \in W^{\prime} \cap T$. It remains to show that $t$ is a canonical generator of $W^{\prime}$.

Supposing to the contrary that $t$ is not a canonical generator, the positive root $\beta_{t}$ is in the positive linear span of $\left\{\beta_{r_{1}}, \beta_{r_{2}}\right\}$, where $r_{1}$ and $r_{2}$ are the canonical generators of $W^{\prime}$. However, $\beta_{t}$ is also in the positive linear span of $\left\{\alpha_{s}: s \in J\right\}$. Since $\beta_{r_{1}}$ and $\beta_{r_{2}}$ are not in $\Phi_{J}$, they are not in the linear span of $\left\{\alpha_{s}: s \in J\right\}$. This contradicts the fact that $\beta_{t}$ has a unique expression as a positive combination of simple roots.

Let $W^{\prime}$ be a generalized rank two parabolic subgroup of $W$ with canonical generators $r_{1}$ and $r_{2}$. We index the reflections of $W^{\prime}$ as

$$
u_{1}=r_{1}, u_{2}=r_{1} r_{2} r_{1}, u_{3}=r_{1} r_{2} r_{1} r_{2} r_{1}, \ldots, u_{m-1}=r_{2} r_{1} r_{2}, u_{m}=r_{2},
$$


where $m=m\left(r_{1}, r_{2}\right)$ is the order of $r_{1} r_{2}$. If $m$ is finite, then the index set is the integers from 1 to $m$. If $m=\infty$, then the index set is the union of the positive integers with the set of formal expressions $\infty-k$, where $k$ is a nonnegative integer. The index set is totally ordered in the obvious way, with the convention that $i<\infty-k$ for any positive integer $i$ and nonnegative integer $k$. The following lemma is the second Proposition of [27], restated in our language:

Lemma 2.17. Let $I$ be a finite subset of $T$. Then the following are equivalent:

(i) There is an element $w$ of $W$ such that $I=\operatorname{inv}(w)$.

(ii) For every generalized parabolic subgroup $W^{\prime}$ of rank two, with canonical generators $r_{1}$ and $r_{2}$, the intersection $I \cap W^{\prime}$ is either an initial or a final segment of $u_{1}, u_{2}, \ldots, u_{m}$.

2.5. Weak order. The (right) weak order on $W$ is the transitive closure of the relations $w<w s$ for all $w \in W$ and $s \in S$ such that $\ell(w)<\ell(w s)$. The weak order is ranked by the length function $\ell$ so that in particular these defining relations are cover relations. Order relations can also be described directly: $v \leq w$ if and only if some (or equivalently every) reduced word for $v$ occurs as a prefix of some reduced word for $w$. There is also a left weak order, with cover relations relating $w$ and $s w$. However, in this paper, all references to the weak order refer to the right weak order. Furthermore, the symbol " $\leq$ " as applied to elements of $W$ always refers to the weak order. The weak order has $w \leq w^{\prime}$ if and only if $\operatorname{inv}(w) \subseteq \operatorname{inv}\left(w^{\prime}\right)$. Thus in particular $x \lessdot y$ implies that $x_{\langle s\rangle}$ is covered by or equal to $y_{\langle s\rangle}$.

Recall that when $\ell(s w)<\ell(w)$, we have $\operatorname{inv}(w)=\{s\} \cup\{s t s: t \in \operatorname{inv}(s w)\}$. As an immediate consequence, we obtain a simple but useful fact about the weak order. For $s \in S$, let $W_{\geq s}$ be the set of elements of $W$ which are above $s$ in the weak order and let $W_{\geq s}$ be $W \backslash W_{\geq s}$.

Proposition 2.18. Let $w \in W$ and $s \in S$. Then $\ell(s w)<\ell(w)$ if and only if $w \geq s$ if and only if $s \in \operatorname{inv}(w)$. Left multiplication by $s$ is an isomorphism of posets from $W_{\geq s}$ to $W_{\geq s}$. If $w^{\prime}>w, w^{\prime} \geq s$ and $w \nsupseteq s$, then $w^{\prime}=s w$.

The following anti-isomorphism result for intervals will also be useful.

Proposition 2.19. For any $w \in W$, the interval $[e, w]$ in the weak order is antiisomorphic to the interval $\left[e, w^{-1}\right]$, via the map $x \mapsto w^{-1} x$.

Proof. Suppose $x \leq y \leq w$. Then there exists a reduced word $a_{1} \cdots a_{k}$ for $w$ and $i$ and $j$ with $i \leq j \leq k$ such that $a_{1} \cdots a_{i}$ is a reduced word for $x$ and $a_{1} \cdots a_{j}$ is a reduced word for $y$. Then $a_{k} a_{k-1} \cdots a_{j+1}$ is a reduced word for $w^{-1} y$, occurring as a prefix of the reduced word $a_{k} a_{k-1} \cdots a_{i+1}$ for $w^{-1} x$, which in turn is a prefix of the reduced word $a_{k} a_{k-1} \cdots a_{1}$ for $w^{-1}$. In particular, the map $x \mapsto w^{-1} x$ is a well-defined order reversing map from $[e, w]$ to $\left[e, w^{-1}\right]$. By swapping $w$ with $w^{-1}$, we see that the inverse map $x \mapsto w x$ is also order reversing.

The weak order is finitary, meaning that for each $w \in W$, the set of elements below $w$ is finite. Weak order on $W$ is also a complete meet-semilattice with a unique minimal element $e$, the identity element of $W$. (Recall that a meet-semilattice is complete if the meet is defined for any nonempty set of elements, even an infinite set.) In particular, any set of elements of $W$ has at most one minimal upper bound (the meet of all upper bounds), and thus any interval in the weak order is a finite lattice. The symbol " $\wedge$ " will refer to the meet in the weak order and the symbol 
" $\vee "$ will refer to the join, when the join exists. An element $j$ in a meet-semilattice is called join-irreducible if any finite set $X$ with $j=\bigvee X$ has $j \in X$. This is equivalent (in a finitary meet-semilattice $L$ ) to the requirement that $j$ covers exactly one element of $L$. (Note that the word "exactly" cannot be replaced by "at most one" here: The only element of $L$ that covers no other element of $L$ is the minimal element $\hat{0}$, which is $\bigvee \emptyset$.) Thus in the case of the weak order on $W$, join-irreducible elements are those which have a unique cover reflection.

When $W$ is finite, the weak order on $W$ is a lattice. In particular, $W$ has a unique maximal element $w_{0}$. This maximal element is an involution. Conjugation by $w_{0}$ permutes $S$.

We now describe the interaction of the weak order with the map $w \mapsto w_{J}$. It is immediate that this map is order preserving. In fact, much more is true. For any $A \subseteq W$ and $J \subseteq S$, let $A_{J}=\left\{w_{J}: w \in A\right\}$. The following result is proven by Jedlička in 22]. (Jedlička denotes the map $w \mapsto w_{J}$ by $\alpha_{J}$. The fact that $\alpha_{J}$ commutes with meet is [22, Lemma 4.2.iii], and the fact that it commutes with join is [22, Lemma 4.5].)

Proposition 2.20. For any $J \subseteq S$ and any subset $A$ of $W$, if $A$ is nonempty, then $\bigwedge\left(A_{J}\right)=(\bigwedge A)_{J}$ and, if $A$ has an upper bound, then $\bigvee\left(A_{J}\right)=(\bigvee A)_{J}$.

Proposition 2.20 has the following simple, but useful, corollary.

Corollary 2.21. Let $x \nsupseteq s$ and $y \nsupseteq s$, and suppose that $x \vee y$ exists. Then $(x \vee y) \nsupseteq s$.

Proof. The assertion that $x \geq s$ is equivalent to the assertion that $x_{\{s\}}$ is the identity element $e$. (Notice that the subscript is $\{s\}$, not $\langle s\rangle$.) If $x_{\{s\}}=e$ and $y_{\{s\}}=e$, then $(x \vee y)_{\{s\}}=x_{\{s\}} \vee y_{\{s\}}=e$ by Proposition 2.20.

We now give two lemmas about cover reflections and the join operation. These are [33, Lemma 2.7] and 33, Lemma 2.8], respectively.

Lemma 2.22. For $w \in W$, if $s$ is a cover reflection of $w$ and every other cover reflection of $w$ is in $W_{\langle s\rangle}$, then $w=s \vee w_{\langle s\rangle}$.

Lemma 2.23. If $w \in W_{\langle s\rangle}$ and $s \vee w$ exists, then $\operatorname{cov}(s \vee w)=\operatorname{cov}(w) \cup\{s\}$.

We now use the weak order to prove several more lemmas about cover reflections, reflection sequences and canonical generators of parabolic subgroups.

Lemma 2.24. Let $w \in W$ and let $J \subseteq S$. If $\operatorname{cov}(w) \subseteq W_{J}$, then $w \in W_{J}$.

Proof. Suppose $w \notin W_{J}$ so that in particular $w_{J}<w$. Choose any $w^{\prime}$ such that $w_{J} \leq w^{\prime} \lessdot w$. Since the map $x \mapsto x_{J}$ is order preserving, $\left(w^{\prime}\right)_{J}=w_{J}$. In particular $\operatorname{inv}\left(w^{\prime}\right) \cap W_{J}=\operatorname{inv}(w) \cap W_{J}$, so that the unique reflection $\operatorname{in} \operatorname{inv}(w) \backslash \operatorname{inv}\left(w^{\prime}\right)$ is not in $W_{J}$. Thus $\operatorname{cov}(w) \nsubseteq W_{J}$.

The following lemma is very close in spirit to [14, Lemma 2.11]. The notation $u_{1}, u_{2}, \ldots, u_{m}$ is explained in the paragraph before Lemma 2.17

Lemma 2.25. Let $t_{1}, t_{2}, \ldots, t_{k}$ be a sequence of distinct reflections in $T$. The following are equivalent:

(i) There is a reduced word $s_{1} s_{2} \cdots s_{k}$ in $W$ such that $t_{1}, t_{2}, \ldots, t_{k}$ is the reflection sequence of $s_{1} s_{2} \cdots s_{k}$. 
(ii) For every generalized parabolic subgroup $W^{\prime}$ of rank two, with canonical generators $r_{1}$ and $r_{2}$, the subsequence of $t_{1}, t_{2}, \ldots, t_{k}$ consisting of elements of $W^{\prime}$ is either an initial or final subsequence of $u_{1}, u_{2}, \ldots, u_{m}$.

Proof. Define $I_{j}=\left\{t_{1}, t_{2}, \ldots, t_{j}\right\}$. First, assume (i) and fix a generalized rank two parabolic subgroup $W^{\prime}$ whose reflections are ordered as in (ii) above. For every $j$ from 1 to $k$, the inversions of $s_{1} s_{2} \cdots s_{j}$ are $I_{j}$ and hence, by Lemma 2.17, $I_{j} \cap W^{\prime}$ is either an initial or final segment of the reflections of $W^{\prime}$. Since this is true for every $I_{j}$, the subsequence of $I$ consisting of reflections in $W^{\prime}$ is either an initial or final subsequence of the reflections of $W^{\prime}$. Now, assume (ii) and fix some $j$ between 1 and $k$. For every rank two generalized parabolic subgroup $W^{\prime}$, the intersection $W^{\prime} \cap I_{j}$ consists of the elements of an initial or final subsequence of the reflections of $W^{\prime}$. So, by Lemma 2.17 again, $I_{j}$ is the inversion set of some element $w_{j}$ of $W$. Then $e, w_{1}, w_{2}, \ldots, w_{k}$ is an ascending saturated chain in the weak order (since the inversion sets are increasing), and thus there is a reduced word $s_{1} s_{2} \cdots s_{k}$ such that $I_{j}=\left\{s_{1}, s_{1} s_{2} s_{1}, \ldots, s_{1} s_{2} \cdots s_{j} \cdots s_{2} s_{1}\right\}$. Then we must have $\left\{s_{1} s_{2} \cdots s_{j} \cdots s_{2} s_{1}\right\}=I_{j} \backslash I_{j-1}=\left\{t_{j}\right\}$, and $t_{1}, t_{2}, \ldots, t_{k}$ is the reflection sequence of $s_{1} s_{2} \cdots s_{k}$, as required.

Lemma 2.25 immediately implies the following:

Lemma 2.26. Let $w \in W$ and let $W^{\prime}$ be a rank two parabolic subgroup, all of whose reflections are in $\operatorname{inv}(w)$. If $t \in W^{\prime}$ is a cover reflection of $w$, then $t$ is a canonical generator of $W^{\prime}$.

Lemma 2.26 and a well-known result show the following.

Lemma 2.27. Let $w \in W$ and let $U=\bigcap_{t \in \operatorname{cov}(w)} H_{t}$. Then the stabilizer of $U$ in $W$ is a finite parabolic subgroup with canonical generators $\operatorname{cov}(w)$.

Proof. Let the cover reflections of $w$ be $t_{1}, t_{2}, \ldots, t_{r}$. Then $t_{i} w=w s_{i}$ for some $s_{i} \in S$; let $J=\left\{s_{1}, \ldots, s_{r}\right\} \subseteq S$. So $U=w V_{J}^{\perp} w^{-1}$. Here $V_{J}^{\perp}$ is the subspace of $V^{*}$ which is orthogonal to the subspace $V_{J}$ of $V$. We claim that the stabilizer of $U$ is $w W_{J} w^{-1}$.

Each generator $t_{i}$ of $w W_{J} w^{-1}$ stabilizes $U$, so the stabilizer of $U$ is contained in $w W_{J} w^{-1}$. Conversely, let $u$ stabilize $U$ and write $u=w v w^{-1}$, so $v$ stabilizes $V_{J}^{\perp}$. In particular, $v$ stabilizes $V_{J}^{\perp} \cap D$, so $v$ is in $W_{J}$ by [21, Theorem 5.13(a)]. We deduce that $u \in w W_{J} w^{-1}$. This concludes the proof that the stabilizer of $U$ is $w W_{J} w^{-1}$.

Now, let $x$ be the minimal length element of $w W_{J}$. The map $v \mapsto x v$ is a poset isomorphism from $W_{J}$ to $w W_{J}$. We assumed that $w$ covers $|J|$ other members of $w W_{J}$ (namely $\left.t_{1} w, \ldots, t_{r} w\right)$, so we conclude that $W_{J}$ has an element which covers $|J|$ other elements. In particular, $W_{J}$ is finite and thus $w W_{J} w^{-1}$ is finite. Furthermore, $w=x w_{0}(J)$, where $w_{0}(J)$ is the maximal element of $W_{J}$. Now, $\operatorname{cov}(w)=w J w^{-1}=x w_{0}(J) J w_{0}(J)^{-1} x^{-1}=x J x^{-1}$. (We have used the fact that conjugation by $w_{0}(J)$ permutes $J$.) By Proposition 2.8 , the canonical generators of $w W_{J} w^{-1}$ are $x J x^{-1}$, since $x$ is a minimal length coset representative of $w W_{J}$. Thus the canonical generators of $w W_{J} w^{-1}$ are the cover reflections of $w$.

2.6. Coxeter elements. A Coxeter element $c$ of $W$ is an element represented by a (necessarily reduced) word $s_{1} s_{2} \cdots s_{n}$ where $S=\left\{s_{1}, \ldots, s_{n}\right\}$ and $n=|S|$. 
Any reduced word for a given Coxeter element $c$ is a permutation of $S$, and typically $c$ has several reduced words. As a special case of Tits' solution to the word problem for Coxeter groups (see e.g. [6, Section 3.3]), any two reduced words for the same Coxeter element are related by a sequence of transpositions of commuting generators. A simple generator $s \in S$ is called initial in $c$ if there is some reduced word for $c$ having $s$ as its first letter. Two different initial letters of the same Coxeter element must commute with each other. A simple generator $s \in S$ is final if it is the last letter of some reduced word for $c$.

The restriction of a Coxeter element $c$ to $W_{J}$ is the Coxeter element of $W_{J}$ obtained by deleting the letters in $S \backslash J$ from some reduced word for $c$. This is independent of the reduced word chosen for $c$.

Several constructions in this paper depend on a choice of $c$, and this dependence is made explicit by superscripts or subscripts in the notation. We often take advantage of the explicit mention of $c$ in this notation to indicate a standard parabolic subgroup. For example, if $c^{\prime}$ is the restriction of $c$ to $W_{J}$, the notation $\pi_{\downarrow}^{c^{\prime}}$ (see Section [6) stands for a map defined on $W_{J}$, not on $W$.

2.7. Sortable elements. Fix a Coxeter element $c$ and a particular word $s_{1} s_{2} \cdots s_{n}$ for $c$ and write a half-infinite word

$$
\left(s_{1} \cdots s_{n}\right)^{\infty}=s_{1} s_{2} \cdots s_{n}\left|s_{1} s_{2} \cdots s_{n}\right| s_{1} s_{2} \cdots s_{n} \mid \cdots
$$

The symbols "|" are inert "dividers" which facilitate the definition of sortable elements. When subwords of $\left(s_{1} \cdots s_{n}\right)^{\infty}$ are interpreted as expressions for elements of $W$, the dividers are ignored. The $\left(s_{1} \cdots s_{n}\right)$-sorting word for $w \in W$ is the lexicographically first (as a sequence of positions in $\left.\left(s_{1} \cdots s_{n}\right)^{\infty}\right)$ subword of $\left(s_{1} \cdots s_{n}\right)^{\infty}$ which is a reduced word for $w$. The $\left(s_{1} \cdots s_{n}\right)$-sorting word can be interpreted as a sequence of subsets of $S$ : Each subset in the sequence is the set of letters of the $\left(s_{1} \cdots s_{n}\right)$-sorting word which occur between two adjacent dividers.

A c-sorting word for $w$ is a word that arises as the $\left(s_{1} \cdots s_{n}\right)$-sorting word for $w$ for some reduced word $s_{1} \cdots s_{n}$ for $c$. Since any two reduced words for $c$ are related by commutation of letters, the $c$-sorting words for $v$ arising from different reduced words for $c$ are related by commutations of letters, with no commutations across dividers. Furthermore, the sequence of subsets defined by a $c$-sorting word is independent of the choice of reduced word $s_{1} \cdots s_{n}$ for $c$. An element $v \in W$ is $c$-sortable if a $c$-sorting word for $v$ defines a sequence of subsets which is weakly decreasing under inclusion.

Example 2.28. Let $W$ be $A_{3}$, the Coxeter group with $S=\{p, q, r\}, m(p, q)=$ $m(q, r)=3$ and $m(p, r)=2$. Let $c=p q r$. Then the $c$-sortable elements are $e, p$, $p q, p q r, p q r p, p q r p q, p q r p q p, p q r q, p q p, p r, q, q r, q r q$ and $r$. There are 14 of these $p q r$-sortable elements; in general, for any Coxeter element $c$ in $A_{n}$, the number of $c$-sortable elements is $\frac{1}{n+2}\left(\begin{array}{c}2 n+2 \\ n+1\end{array}\right)$, the $(n+1)^{\text {st }}$ Catalan number.

We emphasize that sorting words must, by definition, be reduced. The word $p q \mid p q$ may appear to be the $p q r$-sorting word of a $p q r$-sortable element. But, in fact, it is a nonreduced word for the element $q p$, which is not $p q r$-sortable.

Sortable elements satisfy the following recursion, which is easily verified from the definition. 
Proposition 2.29. Let $W$ be a Coxeter group, $c$ a Coxeter element and let $s$ be initial in c. Then an element $w \in W$ is c-sortable if and only if

(i) $w \geq s$, and $s w$ is scs-sortable or

(ii) $w \nsupseteq s$, and $w$ is an sc-sortable element of $W_{\langle s\rangle}$.

Proposition 2.29 amounts to a recursive definition of sortable elements. Recall from Proposition 2.18 that $w \geq s$ is equivalent to the statement that $w$ has a reduced word starting with $s$, which is equivalent to $\ell(s w)<\ell(w)$. Thus in the case $w \geq s$, the definition works by induction on length. In the other case, the induction is on the rank of the Coxeter group. This induction on length and rank is an essential feature of the proofs in this paper.

The following observation is immediate from the definition of sortable elements.

Proposition 2.30. Let $J \subset S$ and let $c^{\prime}$ be the restriction of $c$ to $W_{J}$. Then an element $v \in W_{J}$ is c-sortable if and only if it is $c^{\prime}$-sortable.

\section{The Euler form $E_{c}$ AND the Form $\omega_{c}$}

In this section, we define two bilinear forms, $E_{c}$ and $\omega_{c}$, and establish their basic properties. We then use the form $\omega_{c}$ to characterize the reflection sequences of $c$ sortable elements. We prove some consequences of this characterization. Throughout the section, we fix a symmetrizable choice $A$ of a Cartan matrix for $W$ and a particular choice of the symmetrizing function $\delta$.

We continue to let $s_{1} \cdots s_{n}$ be a reduced word for a Coxeter element $c$. The Euler form $E_{c}$ is defined on the bases of simple roots and co-roots as follows:

$$
E_{c}\left(\alpha_{s_{i}}^{\vee}, \alpha_{s_{j}}\right)= \begin{cases}K\left(\alpha_{s_{i}}^{\vee}, \alpha_{s_{j}}\right)=a_{s_{i} s_{j}} & \text { if } i>j, \\ 1 & \text { if } i=j, \text { or } \\ 0 & \text { if } i<j .\end{cases}
$$

The symmetrization $E_{c}(\alpha, \beta)+E_{c}(\beta, \alpha)$ equals $K(\alpha, \beta)$.

Since all reduced words for $c$ differ only by exchanging commuting generators, the Euler form is independent of the choice of reduced word for $c$. We now present three simple lemmas on the Euler form. The first two follow immediately from the definition.

Lemma 3.1. Let $s$ be initial in $c$ and let $\beta_{t}$ be a positive root. Then $E_{c}\left(\alpha_{s}^{\vee}, \beta_{t}\right)$ equals the coefficient of $\alpha_{s}$ when $\beta_{t}$ is expanded in the basis of simple roots. In particular, $E_{c}\left(\alpha_{s}^{\vee}, \beta_{t}\right) \geq 0$, with equality if and only if $t \in W_{\langle s\rangle}$.

Lemma 3.2. Let $s$ be final in $c$ and let $\beta_{t}^{\vee}$ be a positive co-root. Then $E_{c}\left(\beta_{t}^{\vee}, \alpha_{s}\right)$ equals the coefficient of $\alpha_{s}^{\vee}$ when $\beta_{t}^{\vee}$ is expanded in the basis of simple co-roots. In particular, $E_{c}\left(\beta_{t}^{\vee}, \alpha_{s}\right) \geq 0$, with equality if and only if $t \in W_{\langle s\rangle}$.

Lemma 3.3. If $s$ is initial or final in $c$, then $E_{c}\left(\beta^{\vee}, \beta^{\prime}\right)=E_{s c s}\left(s \beta^{\vee}, s \beta^{\prime}\right)$ for all $\beta$ and $\beta^{\prime}$ in $V$.

Proof. The cases where $s$ is initial and final are equivalent by exchanging the roles of $c$ and scs. We take $s$ to be initial and choose a reduced word for $c$ with $s_{1}=s$. By linearity, it is enough to take $p, q \in S$ and verify the relation for $\beta^{\vee}=\alpha_{p}^{\vee}$ and $\beta^{\prime}=\alpha_{q}$. Then $E_{s c s}\left(s \alpha_{p}^{\vee}, s \alpha_{q}\right)=E_{s c s}\left(\alpha_{p}^{\vee}-a_{p s} \alpha_{s}^{\vee}, \alpha_{q}-a_{s q} \alpha_{s}\right)$, which equals

$$
E_{s c s}\left(\alpha_{p}^{\vee}, \alpha_{q}\right)-E_{s c s}\left(\alpha_{p}^{\vee}, \alpha_{s}\right) a_{s q}-E_{s c s}\left(\alpha_{s}^{\vee}, \alpha_{q}\right) a_{p s}+a_{p s} a_{s q}
$$


If $p \neq s$ and $q \neq s$, then (困) is

$$
E_{s c s}\left(\alpha_{p}^{\vee}, \alpha_{q}\right)-0 \cdot a_{s q}-a_{s q} a_{p s}+a_{p s} a_{s q}=E_{s c s}\left(\alpha_{p}^{\vee}, \alpha_{q}\right)=E_{c}\left(\alpha_{p}^{\vee}, \alpha_{q}\right) .
$$

If $p=s$ and $q \neq s$, then (荬) is $a_{s q}-1 \cdot a_{s q}-a_{s q} \cdot 2+2 \cdot a_{s q}=0=E_{c}\left(\alpha_{p}^{\vee}, \alpha_{q}\right)$. If $p \neq s$ and $q=s$, then (函) is $0-0 \cdot 2-1 \cdot a_{p s}+a_{p s} \cdot 2=a_{p s}=E_{c}\left(\alpha_{p}^{\vee}, \alpha_{q}\right)$. Finally, if $p=q=s$, then (困) is $1-1 \cdot 2-1 \cdot 2+2 \cdot 2=1=E_{c}\left(\alpha_{p}^{\vee}, \alpha_{q}\right)$.

Remark 3.4. The Euler form arises in quiver theory. Lemmas 3.1, 3.2 and 3.3, in the case where $A$ is symmetric and crystallographic, have simple interpretations and proofs in the context of quiver representations. For a brief and accessible introduction to quiver representation theory, see [13. For a thorough introduction which is well suited to our approach, see [2. In particular, see Section III.3 for the homological motivation underlying the Euler form. One thing that is not in [2] is an explanation of the connection between roots and indecomposable representations for a general quiver; for that we recommend the highly readable paper of Kac 24]. Our work is motivated by connections to cluster algebras, as is much current research on quivers; the reader who wishes to know the best current results relating quiver representation theory to cluster algebras should consult [25].

Define a skew-symmetric form $\omega_{c}\left(\beta, \beta^{\prime}\right)=E_{c}\left(\beta, \beta^{\prime}\right)-E_{c}\left(\beta^{\prime}, \beta\right)$. Retaining the fixed reduced word $s_{1} \cdots s_{n}$ for $c$, the form $\omega_{c}$ has the following values on simple roots $\alpha_{s_{i}}$ and $\alpha_{s_{j}}$ :

$$
\omega_{c}\left(\alpha_{s_{i}}, \alpha_{s_{j}}\right)= \begin{cases}K\left(\alpha_{s_{i}}, \alpha_{s_{j}}\right) & \text { if } i>j, \\ 0 & \text { if } i=j, \text { or } \\ -K\left(\alpha_{s_{i}}, \alpha_{s_{j}}\right) & \text { if } i<j .\end{cases}
$$

The form $\omega_{c}$, like the Euler form $E_{c}$, is independent of the choice of reduced word for $c$. In what follows, the sign of $\omega_{c}$, but not its exact value, will be of crucial importance. When $W$ is of rank three, there is a vector $\zeta_{c}$ such that the sign of $\omega_{c}(\alpha, \beta)$ is positive or negative according to whether the angle from $\alpha$ to $\beta$ circles $\mathbb{R} \zeta_{c}$ in a counterclockwise or clockwise direction. See Figures 7, 9 and 13 and the surrounding text. Unlike the Euler form $E_{c}$, which is common in quiver representation theory, and the symmetric form $K$, which occurs in Coxeter theory, Lie theory and elsewhere, the form $\omega_{c}$ seems to be new. The only prior appearances we are aware of are in [35], by the second author, and [26].

Remark 3.5. Since $\beta_{t}^{\vee}$ is a positive scalar multiple of $\beta_{t}$, the quantities $\omega_{c}\left(\beta_{p}, \beta_{q}\right)$ and $\omega_{c}\left(\beta_{p}^{\vee}, \beta_{q}\right)$ always have the same sign. In any statement about the sign of $\omega_{c}$, it is a matter of choice which to write. In this paper, we will prefer the former, because it makes manipulations involving the antisymmetry of $\omega_{c}$ more transparent. However, in cases where the magnitude of $\omega_{c}$ is important, the latter always seems to be the relevant quantity.

Example 3.6. The Coxeter group $A_{n-1}$ is naturally isomorphic to the permutation group $S_{n}$, with simple generators the transpositions $s_{i}=(i i+1)$. The reflections are the transpositions $(i j)$ for $1 \leq i<j \leq n$, with $\beta_{(i j)}=\alpha_{i}+\alpha_{i+1}+\cdots+\alpha_{j-1}$. So, for $1 \leq i<j<k \leq n$, we have $\omega_{c}\left(\beta_{i j}, \beta_{j k}\right)=\omega_{c}\left(\alpha_{j-1}, \alpha_{j}\right)$. So $\omega_{c}\left(\beta_{i j}, \beta_{j k}\right)>0$ if $s_{j-1}$ precedes $s_{j}$ in $c$, and $\omega_{c}\left(\beta_{i j}, \beta_{j k}\right)<0$ if $s_{j}$ precedes $s_{j-1}$. The value of $\omega_{c}$ on other pairs of roots can be worked out similarly. 
The following are the key properties of $\omega_{c}$. The first is immediate from the definition, and the second is immediate from Lemma 3.3. Recall that $V_{J}$ is the real linear span of the parabolic sub root system $\Phi_{J}$.

Lemma 3.7. Let $J \subseteq S$ and let $c^{\prime}$ be the restriction of $c$ to $W_{J}$. Then $\omega_{c}$ restricted to $V_{J}$ is $\omega_{c^{\prime}}$.

Lemma 3.8. If $s$ is initial or final in $c$, then $\omega_{c}\left(\beta, \beta^{\prime}\right)=\omega_{s c s}\left(s \beta, s \beta^{\prime}\right)$ for all roots $\beta$ and $\beta^{\prime}$.

Lemma 3.9. Let $s$ be initial in $c$ and let $t$ be a reflection in $W$. Then $\omega_{c}\left(\alpha_{s}, \beta_{t}\right) \geq 0$, with equality only if $s$ and $t$ commute.

Proof. Expanding in the basis of simple roots, write $\beta_{t}=\sum_{r \in S} a_{r} \alpha_{r}$. Since $\beta_{t}$ is a positive root, all of the $a_{r}$ are nonnegative. So $\omega_{c}\left(\alpha_{s}, \beta_{t}\right)=-\sum_{r \in S \backslash\{s\}} a_{r} K\left(\alpha_{s}, \alpha_{r}\right)$. Each $K\left(\alpha_{s}, \alpha_{r}\right)$ is nonpositive, yielding the required inequality.

Furthermore, $\omega_{c}\left(\alpha_{s}, \beta_{t}\right)=0$ only if $a_{r}=0$ for all $r$ such that $\omega_{c}\left(\alpha_{s}, \alpha_{r}\right) \neq 0$. Thus $\omega_{c}\left(\alpha_{s}, \beta_{t}\right)=0$ only if $t$ is a reflection in the standard parabolic subgroup $W_{J}$, where $J=\left\{s^{\prime}: s s^{\prime}=s^{\prime} s\right\}$. Since $s$ commutes with every generator of $W_{J}$, we know that $s$ commutes with $t$.

A similar proof establishes the following:

Lemma 3.10. Let $s$ be final in $c$ and let $t$ be a reflection in $W$. Then $\omega_{c}\left(\alpha_{s}, \beta_{t}\right) \leq 0$, with strict equality only if $s$ and $t$ commute.

The form $\omega_{c}$ has a special relation with the reflection sequences of $c$-sortable elements. Recall that when $a_{1} \cdots a_{k}$ is a reduced word for some $w \in W$, the reflection sequence associated to $a_{1} \cdots a_{k}$ is $t_{1}, \ldots, t_{k}$, where $t_{i}=a_{1} a_{2} \cdots a_{i} \cdots a_{2} a_{1}$.

Proposition 3.11. Let $a_{1} \cdots a_{k}$ be a reduced word for some $w \in W$ with reflection sequence $t_{1}, \ldots, t_{k}$. Then the following are equivalent:

(i) $\omega_{c}\left(\beta_{t_{i}}, \beta_{t_{j}}\right) \geq 0$ for all $i \leq j$ with strict inequality holding unless $t_{i}$ and $t_{j}$ commute.

(ii) $w$ is c-sortable and $a_{1} \cdots a_{k}$ can be converted to a c-sorting word for $w$ by a sequence of transpositions of adjacent commuting letters.

Figures 13 and 14 illustrate the sequence of partial products of a $c$-sorting word, and the corresponding reflection sequence, in such a way that the truth of condition (i) is visually apparent.

Proof. We prove each implication separately by induction on the rank of $W$ and the length of $w$. The case where either of these is 0 is trivial and serves as our base case. We take advantage of the fact that both (i) and (ii) are unaffected by transpositions of adjacent commuting letters of $a_{1} \cdots a_{k}$.

Suppose (i) holds. Let $s$ be initial in $c$. If $s$ does not occur in the sequence $a_{1} \cdots a_{k}$, then $w \in W_{\langle s\rangle}$. By Lemma 3.7. (i) holds with respect to $s c$ for $w$ and $a_{1} \cdots a_{k}$ in $W_{\langle s\rangle}$, so by induction on rank, $w$ is $s c$-sortable and $a_{1} \cdots a_{k}$ can be converted to an $s c$-sorting word $a_{1}^{\prime} \cdots a_{k}^{\prime}$ for $w$ by transpositions of adjacent commuting letters. By Proposition 2.29, $w$ is $c$-sortable. Furthermore, $a_{1}^{\prime} \cdots a_{k}^{\prime}$ is a $c$-sorting word for $w$.

If $s$ occurs in $a_{1} \cdots a_{k}$, then, if necessary, apply a sequence of transpositions of adjacent commuting letters so as to place the first appearance of $s$ as early as 
possible. Let $a_{1} \cdots a_{k}$ now stand for the new word thus obtained and let $a_{j}$ be the first occurrence of $s$.

Suppose (for the sake of contradiction) that $j>1$. Lemma 2.6 states that $\beta_{t_{j}}$ is in the positive span of $\alpha_{s}$ and $\beta_{t_{i}}$ for $i<j$, say

$$
\beta_{t_{j}}=a \alpha_{s}+\sum_{i=1}^{j-1} b_{i} \beta_{t_{i}}
$$

By hypothesis, $\omega_{c}\left(\beta_{t_{j-1}}, \beta_{t_{j}}\right) \geq 0$. On the other hand,

$$
\omega_{c}\left(\beta_{t_{j-1}}, \beta_{t_{j}}\right)=a \omega_{c}\left(\beta_{t_{j-1}}, \alpha_{s}\right)+\sum_{i=1}^{j-2} b_{i} \omega_{c}\left(\beta_{t_{j-1}}, \beta_{t_{i}}\right) .
$$

By our hypothesis, by Lemma 3.9 and by the antisymmetry of $\omega_{c}$, all of the terms on the right side are nonpositive. Thus $\omega_{c}\left(\beta_{t_{j-1}}, \beta_{t_{j}}\right)=0$, and by the hypothesis again, $t_{j-1}$ and $t_{j}$ commute, so $a_{j-1}$ and $a_{j}$ commute. This contradiction to our choice of $a_{1} \cdots a_{k}$ proves that $j=1$, so $a_{1}=s$.

Now consider the reduced word $a_{2} \cdots a_{k}$ for $s w$. By Lemma 3.8, condition (i) holds for $s w$ and $a_{2} \cdots a_{k}$ with respect to $\omega_{s c s}$. By induction on length, $s w$ is $s c s$-sortable and $a_{2} \cdots a_{k}$ can be converted to an scs-sorting word for $s w$ by transpositions of commuting letters. More specifically, since all scs-sorting words for $s w$ are related by transpositions of commuting letters, $a_{2} \cdots a_{k}$ can be converted to the $\left(s_{2} \cdots s_{n} s_{1}\right)$-sorting word for $s w$, where $s_{1} \cdots s_{n}$ is a reduced word for $c$ with $s_{1}=s$. Prepending $a_{1}$ then gives the $\left(s_{1} \cdots s_{n}\right)$-sorting word for the $c$-sortable element $w$. We have established that (i) implies (ii).

Now suppose (ii) holds. We may as well assume that $a_{1} \cdots a_{k}$ actually is the $\left(s_{1} \cdots s_{n}\right)$-sorting word for $w$, where $s_{1} \cdots s_{n}$ is a reduced word for $c$. Let $s=s_{1}$. If $w \nsupseteq s$, then all the $a_{i}$ and $t_{i}$ are in $W_{\langle s\rangle}$ and all the $\beta_{t_{i}}$ are in $\Phi_{\langle s\rangle}$. By induction on rank, (i) holds with respect to $\omega_{s c}$. By Lemma 3.7. (i) then holds with respect to $\omega_{c}$.

If $w \geq s$, then $a_{1}=s$. Lemma 3.9 says that $\omega_{c}\left(\alpha_{s}, \beta_{t}\right) \geq 0$ for all reflections $t$, with strict inequality unless $s$ and $t$ commute. Thus (i) holds in the case $i=1$. We now consider the case $i>1$. The word $a_{2} \cdots a_{k}$ is the $\left(s_{2} \cdots s_{n} s_{1}\right)$-sorting word for $s w$. For each $j$ with $2 \leq j \leq k$, let $t_{j}^{\prime}=a_{2} a_{3} \cdots a_{j} \cdots a_{3} a_{2}$. By induction on length, $\omega_{s c s}\left(\beta_{t_{i}^{\prime}}, \beta_{t_{j}^{\prime}}\right) \geq 0$ when $2 \leq i<j \leq k$, with strict inequality unless $t_{i}^{\prime}$ and $t_{j}^{\prime}$ commute. Now, since $a_{1} \cdots a_{k}$ is reduced, $s \beta_{t_{j}^{\prime}}=\beta_{s t_{j}^{\prime} s}=\beta_{t_{j}}$ (rather than $-\beta_{t_{j}}$ ). Thus $\omega_{c}\left(\beta_{t_{i}}, \beta_{t_{j}}\right)=\omega_{s c s}\left(\beta_{t_{i}^{\prime}}, \beta_{t_{j}^{\prime}}\right) \geq 0$ when $i<j$, with strict inequality unless $t_{i}$ and $t_{j}$ commute.

Proposition 3.11 leads to a uniform proof (valid for arbitrary $W$ ) of the following lemma [32, Lemma 6.7], which was proved in [32 for finite $W$ by a case-by-case check of the classification of finite Coxeter groups.

Lemma 3.12. Let $s$ be final in $c$ and let $v \in W$ be $c$-sortable. Then $v \geq s$ if and only if $s$ is a cover reflection of $v$.

Proof. The "if" statement is by the definition of cover reflection, so we suppose $v \geq s$ and prove that $s$ is a cover reflection. Let $t_{1}, \ldots, t_{k}$ be the reflection sequence of a $c$-sorting word $a_{1} \cdots a_{k}$ for $v$ and let $i$ be such that $t_{i}=s$. By Proposition 3.11, $\omega_{c}\left(\alpha_{s}, \beta_{t_{j}}\right) \geq 0$ for all $j \geq i$ with strict inequality holding unless $t_{i}$ and $t_{j}$ commute. Lemma 3.10 states that $\omega_{c}\left(\alpha_{s}, \beta_{t}\right) \leq 0$ for any $t$. Thus 
$\omega_{c}\left(\alpha_{s}, \beta_{t_{j}}\right)=0$ for all $j \geq i$, so that $t_{i}$ commutes with $t_{j}$ for all $j \geq i$. Conjugating by $a_{i} \cdots a_{1}$, we see that $a_{i}$ commutes with $a_{i+1} \cdots a_{j} \cdots a_{i+1}$ for all $i \leq j$. By Lemma 2.1, $a_{i}$ commutes with $a_{i+1} \cdots a_{k}$. In particular $v a_{i}<v$, so that $a_{1} \cdots a_{i-1} a_{i+1} \cdots a_{k} a_{i} a_{k} \cdots a_{i+1} a_{i-1} \cdots a_{1}=t_{i}=s$ is a cover reflection of $w$.

Proposition 3.11 also implies the following useful fact.

Proposition 3.13. If $v$ is c-sortable and $J \subseteq S$, then $v_{J}$ is $c^{\prime}$-sortable, where $c^{\prime}$ is the restriction of $c$ to $W_{J}$.

Proof. Suppose $v$ is $c$-sortable and let $t_{1} \cdots t_{k}$ be the reflection sequence of a $c$ sorting word for $v$. Recall that the inversion set of $v_{J} \operatorname{is} \operatorname{inv}(v) \cap W_{J}$. Order $\operatorname{inv}\left(v_{J}\right)$ by restricting the ordering $t_{1} \cdots t_{k}$. Lemmas 2.16 and 2.25imply that the restricted order is the reflection sequence for a reduced word $a_{1} \cdots a_{m}$ for $v_{J}$. Lemma 3.7 and Proposition 3.11 imply that $a_{1} \cdots a_{m}$ can be converted, by a sequence of transpositions of adjacent commuting letters, to a $c^{\prime}$-sorting word for $v_{J}$.

\section{Orientation and alignment}

In this section, we relate Proposition 3.11 to the notions of $c$-orientation and $c$-alignment defined in 32. To understand the essential idea behind orientation and alignment, consider the example of the rank two Coxeter group $W$ of type $B_{2}$, with $S=\left\{s_{1}, s_{2}\right\}$ and $m\left(s_{1}, s_{2}\right)=4$. By Lemma 2.17, the inversion set of any element of $W$ is either an initial segment or a final segment of the sequence $s_{1}, s_{1} s_{2} s_{1}, s_{2} s_{1} s_{2}, s_{2}$. The inversion sets of the $s_{1} s_{2}$-sortable elements are

$$
\emptyset,\left\{s_{1}\right\},\left\{s_{1}, s_{1} s_{2} s_{1}\right\},\left\{s_{1}, s_{1} s_{2} s_{1}, s_{2} s_{1} s_{2}\right\},\left\{s_{1}, s_{1} s_{2} s_{1}, s_{2} s_{1} s_{2}, s_{2}\right\} \text {, and }\left\{s_{2}\right\} \text {. }
$$

Notice that if the inversion set of an $s_{1} s_{2}$-sortable element contains more than one reflection, it must be an initial segment of $s_{1}, s_{1} s_{2} s_{1}, s_{2} s_{1} s_{2}, s_{2}$. By symmetry, the inversion sets of $s_{2} s_{1}$-sortable elements containing more than one reflection must be final segments of $s_{1}, s_{1} s_{2} s_{1}, s_{2} s_{1} s_{2}, s_{2}$. Thus the choice of Coxeter element specifies an orientation of the reflections in the rank two Coxeter group.

The content of [32, Section 3] is that for any finite Coxeter group $W$, the choice of a Coxeter element specifies an orientation, with certain desirable properties, of each rank two parabolic subgroup of $W$. The content of [32, Theorem 4.1] is that an element $w \in W$ is $c$-sortable if and only if it is $c$-aligned, meaning that, for any rank two parabolic subgroup $W^{\prime}$, if the intersection $\operatorname{inv}(w) \cap W^{\prime}$ has more than one element, it respects the $c$-orientation of $W^{\prime}$ in the sense of the $B_{2}$ example above.

Theorem [4.3, below, generalizes [32, Theorem 4.1] to the case where $W$ is not necessarily finite. The proof of Theorem 4.3 specializes to an alternate proof of [32, Theorem 4.1] without resorting to type-by-type arguments. Theorem 4.3 is a strengthening of Proposition 3.11 which provides a more pleasant characterization of sortability: It characterizes a sortable element by its inversion set, rather than by the requirement that its inversion sets can be totally ordered in a special way.

The statement of Theorem 4.3 differs in notation from the statement of 32 , Theorem 4.1]. In [32, $c$-orientations are described as a directed cycle structure on the reflections of each rank two parabolic subgroup $W$, and [32, Theorem 4.1] is phrased in terms of the oriented cycle structure. The oriented cycles of [32] are natural in light of the combinatorial models for $c$-orientation introduced in [32, Section 3]. In the present context, it is more reasonable to totally order the reflections of $W^{\prime}$ based on the sign of $\omega_{c}$ applied to the corresponding positive roots 
and to phrase Theorem 4.3 in terms of the total orders. The interested reader will easily see the relationship between the directed cycles of [32] and the total orders used here.

Let $W^{\prime}$ be a generalized rank two parabolic subgroup of $W$ with canonical generators $r_{1}$ and $r_{2}$, and continue to index the reflections of $W^{\prime}$ as $u_{1}, u_{2}, \ldots, u_{m}$ as explained in the paragraph before Lemma 2.17

Proposition 4.1. Let $W^{\prime}$ be a generalized rank two parabolic subgroup with reflections indexed as above.

(i) If $\omega_{c}\left(\beta_{u_{1}}, \beta_{u_{m}}\right)=0$, then $\omega_{c}\left(\beta_{u_{i}}, \beta_{u_{j}}\right)=0$ for all indices $i$ and $j$.

(ii) If $\omega_{c}\left(\beta_{u_{1}}, \beta_{u_{m}}\right)<0$, then $\omega_{c}\left(\beta_{u_{i}}, \beta_{u_{j}}\right)<0$ for all indices $i<j$.

(iii) If $\omega_{c}\left(\beta_{u_{1}}, \beta_{u_{m}}\right)>0$, then $\omega_{c}\left(\beta_{u_{i}}, \beta_{u_{j}}\right)>0$ for all indices $i<j$.

When (i) applies, we say that the restriction of $\omega_{c}$ to $W^{\prime}$ is zero.

Proof. Assertion (i) is immediate from the fact that $\omega_{c}$ is linear and skew-symmetric and the fact that all positive roots associated to reflections in $W^{\prime}$ are in the positive span of $\beta_{u_{1}}$ and $\beta_{u_{m}}$.

Since $u_{1}$ and $u_{m}$ are the canonical generators of $W^{\prime}$, we can write $\beta_{u_{i}}=a \beta_{u_{1}}+$ $b \beta_{u_{m}}$ and $\beta_{u_{j}}=c \beta_{u_{1}}+d \beta_{u_{m}}$ for nonnegative scalars $a, b, c$ and $d$ with $a+b>0$ and $c+d>0$. Both assertions (ii) and (iii) follow from the assertion that $a d-b c>0$. The latter is immediate in the case where $i=1$ or $j=m$. If neither $i=1$ nor $j=m$, then write $u_{1}\left(\beta_{u_{i}}\right)=\beta_{u_{i^{\prime}}}$ for some $i^{\prime}$ and $u_{1}\left(\beta_{u_{j}}\right)=\beta_{u_{j^{\prime}}}$ for some $j^{\prime}$. One easily verifies that $i^{\prime}>j^{\prime}$ and, similarly, that the action of $u_{m}$ on $\beta_{u_{i}}$ and $\beta_{u_{j}}$ reverses the total order on $u_{2}, \ldots, u_{m-1}$. One also easily checks that the action of $u_{1}$ or $u_{m}$ reverses the sign of $a d-b c$. Acting a finite number of times by $u_{1}$ and $u_{m}$, we eventually reach the case where $i=1$ or $j=m$, thus completing the proof.

We are now prepared to state the main result of this section. Let $W^{\prime}$ be a noncommutative generalized rank two parabolic subgroup of $W$ and let $w$ be an element of $W$. We say that $w$ is c-aligned with respect to $W^{\prime}$ if one of the following cases holds:

(1) Indexing the reflections as above, $\omega_{c}\left(\beta_{u_{i}}, \beta_{u_{j}}\right)>0$ for all indices $i<j$, and $\operatorname{inv}(w) \cap W^{\prime}$ is either the empty set, the singleton $\left\{u_{m}\right\}$ or a (necessarily finite) initial segment of $u_{1}, u_{2}, \ldots, u_{m}$.

(2) The restriction of $\omega_{c}$ to $W^{\prime}$ is zero, and $\operatorname{inv}(w) \cap W^{\prime}$ is either the empty set or a singleton.

We say that $w$ is $c$-aligned if $w$ is $c$-aligned with respect to every noncommutative rank two parabolic subgroup of $W$. As we will see below, this implies that $w$ is also $c$-aligned with respect to every generalized noncommutative rank two parabolic subgroup.

Example 4.2. We describe $c$-aligned elements in type $A_{n-1}$, relying on the computations from Example 3.6. Let $W^{\prime}$ be a noncommutative rank two parabolic subgroup. $W^{\prime}$ must be of type $A_{2}$, with canonical generators $(i j)$ and $(j k)$ for some $1 \leq i<j<k \leq n$. Suppose that $s_{j-1}$ precedes $s_{j}$ in $c$. Then, as we saw in Example 3.6, we have $\omega_{c}\left(\beta_{(i j)}, \beta_{(j k)}\right) \geq 0$. In the notation above, the reflections in this rank two parabolic are $\left(u_{1}, u_{2}, u_{3}\right)=((i j),(i k),(j k))$. So an element $w \in A_{n-1}$ is $c$-aligned with respect to $W^{\prime}$ if and only if $\operatorname{inv}(w) \cap W^{\prime} \neq\{(i k),(j k)\}$.

In terms of the one-line representation of the permutation $w$, this says that $w$ avoids $k i j$. Similarly, if $s_{j}$ precedes $s_{j-1}$ in $c$, then $w$ must avoid $j k i$. This recovers 
the pattern avoidance description of alignment from 32. It is similarly easy in this way to recover the pattern avoidance descriptions of alignment in the other classical finite types, and produce new pattern avoidance descriptions in the classical affine types. In the exceptional types, there is no simple description of $W$ in terms of permutations. However, the uniform definition of alignment in terms of $\omega_{c}$ is a dramatic improvement over the computer-aided definition in [32, Sections 3-4].

For a geometric depiction of the inversion set of a $c$-aligned element, see the discussion surrounding Figure 13 .

Theorem 4.3. An element $w$ of $W$ is c-sortable if and only if it is c-aligned. In this case, $w$ is also c-aligned with respect to every generalized noncommutative rank two parabolic subgroup of $W$.

Proof. The fact that $c$-sortability implies $c$-alignment follows immediately from Proposition 3.11 and Lemma 2.17. The same argument also shows that $c$-sortability implies alignment with respect to all generalized noncommutative rank two parabolic subgroups.

Suppose $w$ is $c$-aligned. Let $s$ be initial in $c$, and first consider the case where $s \leq w$. Let $W^{\prime}$ be a noncommutative parabolic subgroup of rank two with canonical generators $r_{1}$ and $r_{2}$. By hypothesis, $w$ is $c$-aligned with respect to $W^{\prime}$. We claim that $s w$ is $s c s$-aligned with respect to $s W^{\prime} s$. If $r_{1}, r_{2} \neq s$, then the claim is immediate by Lemmas 2.15 and 3.8. Otherwise, take $r_{1}=s$ without loss of generality. In this case, $s W^{\prime} s=W^{\prime}$. Since $w \geq s$, we know that $s w \geq s$ and $s$ is not an inversion of $s w$. So, by Lemma 2.17, $\operatorname{inv}(s w) \cap W^{\prime}$ is an initial segment of $r_{2}, r_{2} s r_{2}$, $r_{2} s r_{2} s r_{2} \ldots$ Since $W^{\prime}$ is noncommutative, $\omega_{s c s}\left(\beta_{r_{2}}, \alpha_{s}\right)>0$ by Lemma 3.9 and Proposition 4.1, and we see that $s w$ is $s c s$-aligned with respect to $W^{\prime}$. Letting $W^{\prime}$ vary over all noncommutative rank two parabolic subgroups, we see that $s w$ is scs-aligned with respect to every noncommutative rank two parabolic subgroup, or in other words that $s w$ is $s c s$-aligned. By induction on $\ell(w)$, the element $s w$ is scs-sortable, so $w$ is $c$-sortable by Proposition 2.29.

Now consider the case where $s \not \leq w$. We first show that $w \in W_{\langle s\rangle}$. Suppose to the contrary that $w \notin W_{\langle s\rangle}$, so that in particular, $w_{\langle s\rangle}<w$. By Lemmas 2.16 and 3.7 we see that $w_{\langle s\rangle}$ is $s c$-aligned, so it is $s c$-sortable by induction on the rank of $W$ and therefore $c$-sortable by Proposition 2.30, Let $a_{1} \cdots a_{j}$ be a $c$-sorting word for $w_{\langle s\rangle}$. Since $w_{\langle s\rangle}<w$, we can extend this word to a reduced word $a_{1} \cdots a_{k}$ for $w$ for some $k>j$. Note that $a_{j+1}=s$, because otherwise $a_{1} \cdots a_{j+1}$ is a reduced word for an element of $W_{\langle s\rangle}$ which is greater than $w$, contradicting the definition of $w_{\langle s\rangle}$. For each $i \in[k]$, let $t_{i}$ be the reflection given by $a_{1} \cdots a_{i} \cdots a_{1}$ and let $r_{i}=a_{1} \cdots a_{i} s a_{i} \cdots a_{1}$. We claim that $r_{i}$ is an inversion of $w$ for each $i$ with $0 \leq i \leq j$.

The claim is proven by induction on $j-i$, with the base case $j-i=0$ given by the fact that $r_{j}=t_{j+1}$. Suppose $0 \leq i<j$. The reflection $t_{i}$ is an inversion of $w$, and by induction $r_{i+1}$ is also an inversion of $w$. The rank two parabolic subgroup $W^{\prime}=$ $a_{1} \cdots a_{i} W_{\left\{a_{i+1}, s\right\}} a_{i} \cdots a_{1}$ contains $r_{i}, r_{i+1}$ and $t_{i+1}$. The word $a_{1} \cdots a_{i+1}$ is reduced because it is a prefix of a reduced word. The word $a_{1} \cdots a_{i}$ is reduced for the same reason, and thus $a_{1} \cdots a_{i} s$ is reduced because the letter $s$ does not occur in $a_{1} \cdots a_{i}$. Thus $a_{1} \cdots a_{i}$ is a minimal length coset representative for $a_{1} \cdots a_{i} W_{\left\{a_{i+1}, s\right\}}$, so the canonical generators of $W^{\prime}$ are $t_{i+1}$ and $r_{i}$ by Proposition 2.8. If $t_{i+1}$ and $r_{i}$ commute, then $a_{i+1}$ and $s$ commute, so that the claim holds because $r_{i}=r_{i+1}$. We 
may thus assume that $t_{i+1}$ and $r_{i}$ do not commute. In particular, $r_{i+1}=t_{i+1} r_{i} t_{i+1}$ is distinct from $t_{i+1}$ and $r_{i}$.

Since $a_{1} \cdots a_{i+1}$ is a reduced word for an element of $W_{\langle s\rangle}$, by Lemma 2.6 we conclude that $\beta_{r_{i+1}}$ is in the positive span of $\alpha_{s}$ and $\left\{\beta_{t_{l}}: l \leq i+1\right\}$, say $\beta_{r_{i+1}}=$ $a \alpha_{s}+\sum_{l=1}^{i+1} b_{l} \beta_{t_{l}}$. Thus

$$
\omega_{c}\left(\beta_{t_{i+1}}, \beta_{r_{i+1}}\right)=a \omega_{c}\left(\beta_{t_{i+1}}, \alpha_{s}\right)+\sum_{l=1}^{i+1} b_{l} \omega_{c}\left(\beta_{t_{i+1}}, \beta_{t_{l}}\right) .
$$

By Lemma 3.9 and the antisymmetry of $\omega_{c}$, the first term on the right side is nonpositive. Since $a_{1} \cdots a_{j}$ is a $c$-sorting word for a $c$-sortable element, Proposition 3.11 and the antisymmetry of $\omega_{c}$ imply that the remaining terms on the right side are also nonpositive. Furthermore, if every term of the right side is zero, then $t_{i+1}$ commutes with each $t_{l}$ with $l \leq i$, and $s$ commutes with $t_{i+1}$. If $t_{i+1}$ commutes with each $t_{l}$ with $l \leq i$, then one easily concludes that $a_{i+1}$ commutes with each $a_{l}$ with $l \leq i$ and that $t_{i+1}=a_{i+1}$. If in addition $s$ commutes with $t_{i+1}=a_{i+1}$, then $t_{i+1}$ and $r_{i}$ commute, contradicting our assumption. Thus $\omega_{c}\left(\beta_{t_{i+1}}, \beta_{r_{i+1}}\right)<0$. Applying Proposition 4.1, we conclude that $\omega_{c}\left(\beta_{t_{i+1}}, \beta_{r_{i}}\right)<0$. Since $w$ is $c$-aligned and its inversion set contains $t_{i+1}$ and $r_{i+1}$, the inversion set of $w$ must also contain $r_{i}$. This proves the claim.

The claim for $i=0$ is the statement that $s \leq w$, contradicting the assumption that $s \not \leq w$. This contradiction proves that $w \in W_{\langle s\rangle}$. It is immediate by Lemmas 2.16 and 3.7 that $w$ is $s c$-aligned as an element of $W_{\langle s\rangle}$. By induction on rank, $w$ is $s c$-sortable, and thus is $c$-sortable by Proposition 2.29 .

Remark 4.4. A consequence of Theorem 4.3 is that, if $w$ is any $c$-aligned element, then we can order $\operatorname{inv}(w)$ as $t_{1}, t_{2}, \ldots, t_{k}$ such that $\omega\left(t_{i}, t_{j}\right) \geq 0$ whenever $i<j$. If we knew that $\operatorname{inv}(w)$ could be so ordered, even when $w$ is not $c$-sortable, then we could establish Theorem 4.3 as follows: By Lemma 2.25, $t_{1}, t_{2}, \ldots, t_{k}$ is the reflection sequence of some reduced word for $w$. (This step uses the hypothesis that $w$ is aligned.) By Proposition 3.11, that reduced word is the sorting word for a sortable element. Unfortunately, $\operatorname{inv}(w)$ cannot always be so ordered, although counterexamples are quite large and occur only in infinite groups.

Remark 4.5. When $W$ is finite, case (2) in the definition of $c$-alignment cannot happen. (Indeed, if case (2) occurred, then the present definition of alignment would not agree with the definition in [32, Theorem 4.1].) One way to see that case (2) cannot occur when $W$ is finite is to appeal to Corollary 7.2. which says that $w_{0}$ is $c$-sortable, and thus $c$-aligned by Theorem 4.3. Since $\operatorname{inv}\left(w_{0}\right)$ is all of $T$, in particular $\operatorname{inv}\left(w_{0}\right) \cap W^{\prime}$ cannot be the empty set or a singleton.

\section{FORCED AND UNFORCED SKIPS}

In this section, we define a set $C_{c}(v)$ of $n$ roots associated to each sortable element $v$, and we prove several key properties of $C_{c}(v)$. In Section 6 we define and study the $c$-Cambrian cone $\operatorname{Cone}_{c}(v)$ whose inward normals are $C_{c}(v)$. The main results of the present section are characterizations of $C_{c}(v)$ in terms of "skips" in a $c$-sorting word for $v$ and in terms of cover reflections of $v$. 
Let $v$ be $c$-sortable and let $r \in S$ be a simple reflection. We define a root $C_{c}^{r}(v)$ inductively as follows. For $s$ initial in $c$,

$$
C_{c}^{r}(v)= \begin{cases}\alpha_{r} & \text { if } v \nsupseteq s \text { and } r=s, \\ C_{s c}^{r}(v) & \text { if } v \nsupseteq s \text { and } r \neq s, \\ s C_{s c s}^{r}(s v) & \text { if } v \geq s .\end{cases}
$$

In the second case listed above, the root $C_{s c}^{r}(v)$ is defined in $W_{\langle s\rangle}$ by induction on rank, and in the third case, $C_{s c s}(s v)$ is defined by induction on length. Implicitly, this recursive description depends on a choice of initial element for $c$, but it is easy to check that $C_{c}^{r}(v)$ is, in fact, well defined. See Lemma 6.6 for a detailed discussion of a similar issue.

Let $C_{c}(v)=\left\{C_{c}^{r}(v): r \in S\right\}$. In other words,

$$
C_{c}(v)= \begin{cases}C_{s c}(v) \cup\left\{\alpha_{s}\right\} & \text { if } v \nsupseteq s, \\ s C_{s c s}(s v) & \text { if } v \geq s .\end{cases}
$$

An easy inductive argument shows that $C_{c}(v)$ is a basis of $V$ and, in the crystallographic case, of the root lattice.

We define $A_{c}(v)=C_{c}(v) \cap \Phi^{-}$and $B_{c}(v)=C_{c}(v) \cap \Phi^{+}$. We now give a combinatorial description of $A_{c}(v)$ and $B_{c}(v)$ in terms of a $c$-sorting word for $v$.

Let $w$ be an element of $W$, let $s_{1} \cdots s_{n}$ be a reduced word for $c$, let $a_{1} \cdots a_{k}$ be the $\left(s_{1} \cdots s_{n}\right)$-sorting word for $w$ and let $r \in S$. Recall the definition of the $\left(s_{1} \cdots s_{n}\right)$ sorting word as the leftmost subword of $\left(s_{1} \cdots s_{n}\right)^{\infty}$ that is a reduced word for $w$. Since $w$ is of finite length, there is a leftmost instance of $r$ in $\left(s_{1} \cdots s_{n}\right)^{\infty}$ that is not in that subword. Let $i$ be such that this leftmost instance of $r$ in $\left(s_{1} \cdots s_{n}\right)^{\infty}$ occurs (unless $i=0$ ) after the location of $a_{i}$ and (unless $i=k$ ) before the location of $a_{i+1}$. Say $a_{1} \cdots a_{k}$ skips $r$ in the $(i+1)^{\text {st }}$ position. If $a_{1} \cdots a_{i} r$ is reduced, then this is an unforced skip; otherwise it is a forced skip.

For any $w \in W$, define

$\mathrm{fs}_{c}(w)=\left\{a_{1} \cdots a_{i} r a_{i} \cdots a_{1}: a_{1} \cdots a_{k}\right.$ has a forced skip of $r$ in position $\left.i+1\right\}$, $\operatorname{ufs}_{c}(w)=\left\{a_{1} \cdots a_{i} r a_{i} \cdots a_{1}: a_{1} \cdots a_{k}\right.$ has an unforced skip of $r$ in position $\left.i+1\right\}$.

The sets $\mathrm{fs}_{c}(w)$ and $\mathrm{ufs}_{c}(w)$ are easily seen to be independent of the choice of reduced word $s_{1} \cdots s_{n}$ for $c$, but they do, of course, depend on the choice of $c$.

The main results of this section are the following four propositions. For examples illustrating these results, see Section 10.1. Recall that $\operatorname{cov}(w)$ stands for the set of cover reflections of an element $w \in W$.

Proposition 5.1. Let $v$ be c-sortable with c-sorting word $a_{1} \cdots a_{k}$ and let $r \in S$. Let $a_{1} \cdots a_{k}$ skip $r$ in the $(i+1)^{\text {st }}$ position and let $t=a_{1} \cdots a_{i} r a_{i} \cdots a_{1}$. Then $C_{c}^{r}(v)= \pm \beta_{t}$. Furthermore,

$$
\begin{aligned}
A_{c}(v) & =\left\{C_{c}^{r}(v): \text { the skip of } r \text { in } a_{1} \cdots a_{k} \text { is forced }\right\} \\
& =\left\{-\beta_{t}: t \in \mathrm{fs}_{c}(v)\right\}, \text { and } \\
B_{c}(v) & =\left\{C_{c}^{r}(v): \text { the skip of } r \text { in } a_{1} \cdots a_{k} \text { is unforced }\right\} \\
& =\left\{\beta_{t}: t \in \operatorname{ufs}_{c}(v)\right\} .
\end{aligned}
$$

Proposition 5.2. Let $v$ be c-sortable. Then $A_{c}(v)=\left\{-\beta_{t}: t \in \operatorname{cov}(v)\right\}$. 
Proposition 5.3. Let $s$ be final in $c$ and let $v$ be c-sortable with $v \geq s$. Then

(i) $v=s \vee v_{\langle s\rangle}$,

(ii) $\operatorname{cov}(v)=\{s\} \cup \operatorname{cov}\left(v_{\langle s\rangle}\right)$, and

(iii) $\operatorname{ufs}_{c}(v)=\operatorname{ufs}_{c s}\left(v_{\langle s\rangle}\right)$.

Proposition 5.4. Let $s$ be initial in $c$ and let $v$ be a c-sortable element of $W$ such that $s$ is a cover reflection of $v$. Then

(i) $v=s \vee v_{\langle s\rangle}$,

(ii) $\operatorname{cov}(v)=\{s\} \cup \operatorname{cov}\left(v_{\langle s\rangle}\right)$, and

(iii) $\operatorname{ufs}_{c}(v)=\left\{s t s: t \in \operatorname{ufs}_{s c}\left(v_{\langle s\rangle}\right)\right\}$.

Example 5.5. Let $W$ be $\tilde{A}_{2}$, the rank three Coxeter group with $S=\{p, q, r\}$ and $m(p, q)=m(p, r)=m(q, r)=3$. Take $c=p q r$ and consider $v=p q r p r$. This word is reduced, so $v$ is $c$-sortable. The letter $q$ is skipped in the 5 th position, and the letters $p$ and $r$ in the 6th. So Proposition [5.1 states that $C_{c}^{q}(v)=p q r p \alpha_{q}=\beta_{p q r p q p r q p}$, $C_{c}^{p}(v)=\operatorname{pqrpr}_{p}=-\beta_{p q r q p}$ and $C_{c}^{r}(v)=\operatorname{pqrpr}_{r}=-\beta_{q}$. The reader who checks this from the recursive definition of $C_{v}$ will quickly see why Proposition 5.1 is true. The reflection pqrpqprqp is an unforced skip, while pqrqp and $q$ are forced. Proposition 5.2 states that pqrqp and $q$ are the cover reflections of $v$.

The proofs of the latter two results require the Euler form, and thus a choice of symmetrizable Cartan matrix. However, the results themselves are strictly combinatorial, not geometric. These combinatorial results restrict the geometry of those cones of the Cambrian fan which have a wall contained in $H_{s}$, as explained in Proposition 9.7 and Section 10. See Figures 3 and 4 and the surrounding discussion. We first prepare to prove Propositions 5.1 and 5.2 by proving some preliminary lemmas.

Lemma 5.6. If $-\beta_{t} \in A_{c}(v)$, then $t$ is an inversion of $v$. If $\beta_{t} \in B_{c}(v)$, then $t$ is not an inversion of $v$.

Proof. We prove the equivalent statement

$$
C_{c}(v) \cap\left\{ \pm \beta_{t}: t \in \operatorname{inv}(v)\right\}=A_{c}(v) .
$$

Let $s$ be initial in $c$. If $v \ngtr s$, then $C_{c}(v)=C_{s c}(v) \cup\left\{\alpha_{s}\right\}$. By induction on rank, $C_{s c}(v) \cap\left\{ \pm \beta_{t}: t \in \operatorname{inv}(v)\right\}=A_{s c}(v)$. This completes the argument in this case since $s \notin \operatorname{inv}(v)$ and $A_{s c}(v)=A_{c}(v)$.

If $v \geq s$, then $C_{c}(v)=s C_{s c s}(s v)$ and $\operatorname{inv}(s v)=(s \operatorname{inv}(v) s) \backslash\{s\}$. By induction on length, $C_{s c s}(s v) \cap\left\{ \pm \beta_{t}: t \in \operatorname{inv}(s v)\right\}=A_{s c s}(s v)$. Thus

$$
C_{c}(v) \cap\left\{ \pm \beta_{t}: t \in \operatorname{inv}(v), t \neq s\right\}=s A_{s c s}(s v)=A_{c}(v) \backslash\left\{-\alpha_{s}\right\} .
$$

So we just need to consider whether $\pm \alpha_{s}$ could be contained in either side of equation (买). If $-\alpha_{s} \in C_{c}(v)$, then $A_{c}(v)=s A_{s c s}(s v) \cup\left\{-\alpha_{s}\right\}$; otherwise $A_{c}(v)=$ $s A_{s c s}(s v)$. So $-\alpha_{s}$ is contained in the right hand side of equation (疋) if and only if it is contained in the left hand side. We now check that $\alpha_{s}$ cannot lie in the left hand side; by definition, $\alpha_{s}$ cannot belong to $A_{c}(v)$. If $\alpha_{s}$ was in $C_{c}(v)$, then $-\alpha_{s}$ would be in $C_{s c s}(s v)$ and hence $-\alpha_{s} \in A_{s c s}(s v)$. So, by induction on length, $s$ would be an inversion of $s v$, which contradicts our assumption that $v \geq s$.

\footnotetext{
${ }^{4}$ Note that the use of the notation $E \backslash F$ does not imply $F \subset E$. Here and throughout the paper, the definition of $E \backslash F$ is $\{x \in E: x \notin F\}$.
} 
Lemma 5.7. Let $w \in W$ have c-sorting word $a_{1} \cdots a_{k}$. If $a_{1} \cdots a_{k}$ skips $r$ in the $(i+1)^{\mathrm{st}}$ position, then the skip is forced if and only if $a_{1} \cdots a_{i} r a_{i} \cdots a_{1} \in \operatorname{inv}(w)$.

Proof. Suppose $a_{1} \cdots a_{k}$ skips $r$ in the $(i+1)^{\text {st }}$ position. Set $t=a_{1} \cdots a_{i} r a_{i} \cdots a_{1}$ and $t_{j}=a_{1} \cdots a_{j} \cdots a_{1}$ for each $j$ with $1 \leq j \leq k$. Since $a_{1} \cdots a_{i}$ is reduced, the word $a_{1} \cdots a_{i} r$ is reduced if and only if $t \neq t_{j}$ for every $j$ with $1 \leq j \leq i$. However, if $t=t_{j}$ for some $i<j \leq k$, then skipping $r$ in the $(i+1)^{\text {st }}$ position violates the definition of a $c$-sorting word. Thus $a_{1} \cdots a_{i} r$ is reduced (and thus the skip is unforced) if and only if $a_{1} \cdots a_{i} r a_{i} \cdots a_{1} \notin \operatorname{inv}(w)$.

Lemma 5.8. Let $v$ be a c-sortable element of $W$. Suppose a c-sorting word $a_{1} \cdots a_{k}$ for $v$ has an unforced skip of the generator $r$ at the $(i+1)^{\text {st }}$ position. Let $t=$ $a_{1} \cdots a_{i} r a_{i} \cdots a_{1}$, and for each $j \in[k]$ define $t_{j}=a_{1} \cdots a_{j} \cdots a_{1}$. Then

(1) $a_{1} \cdots a_{i} r$ is a c-sorting word for a c-sortable element.

(2) $r \neq a_{j}$ for every $j$ with $i<j \leq k$.

(3) $\omega_{c}\left(\beta_{t}, \beta_{t_{j}}\right) \geq 0$ for every $j$ with $i<j \leq k$, with strict equality holding unless $t$ and $t_{j}$ commute.

Proof. Let $c=s_{1} s_{2} \cdots s_{n}$ and let $a_{1} a_{2} \cdots a_{k}$ be the $\left(s_{1} \cdots s_{n}\right)$-sorting word for $v$.

The first two assertions are immediate from the relevant definitions. For the last assertion, we argue by induction on length and rank. Let $s=s_{1}$. First suppose $v \nsupseteq s$. If $r=s$, then Lemma 3.9 proves the assertion. If not, then $v \in W_{\langle s\rangle}$ is $s c$-sortable, $a_{1} \cdots a_{k}$ is an $s c$-sorting word and the assertion follows by induction on rank and by Lemma 3.7

On the other hand, if $v \geq s$, then in particular $a_{1}=s$, so an $s c s$-sorting word $a_{2} \cdots a_{k}$ for $s v$ has an unforced skip of $r$ at position $i$. By induction of length for $t^{\prime}=a_{2} \cdots a_{i} r a_{i} \cdots a_{2}$ and $t_{j}^{\prime}=a_{2} \cdots a_{j} \cdots a_{2}$ we have $\omega_{s c s}\left(\beta_{t^{\prime}}, \beta_{t_{j}^{\prime}}\right) \geq 0$ for all $j$ with $i<j \leq k$, with strict equality holding unless $t$ and $t_{j}$ commute. Now apply Lemma 3.8

We now prove Propositions 5.1 and 5.2

Proof of Proposition 5.1. We will verify the statement $C_{c}^{r}(v)= \pm \beta_{t}$ by induction on length and rank. The statements about $A_{c}$ and $B_{c}$ follow by Lemmas 5.6 and 5.7. Let $a_{1} \cdots a_{k}$ be the $\left(s_{1} \cdots s_{n}\right)$-sorting word for $v$ and let $s=s_{1}$.

If $v \nsucceq s$, then consider the cases $r=s$ and $r \neq s$. If $r=s$, then $t=s$ and $C_{c}^{s}(v)=\alpha_{s}$. If $r \neq s$, then $C_{c}^{r}(v)=C_{s c}^{r}(v)$, which equals $\pm \beta_{t}$ by induction on rank.

If $v \geq s$, then $C_{c}^{r}(v)=s C_{s c s}^{r}(s v)$. An $s c s$-sorting word for $s v$ can be obtained from $a_{1} \cdots a_{k}$ by deleting the initial $s=a_{1}$. Thus $s v$ skips $r$ in the $i^{\text {th }}$ position and $a_{2} \cdots a_{i} r a_{i} \cdots a_{2}=s t s$. By induction on the length of $v, C_{s c s}^{r}(s v)= \pm \beta_{s t s}$, so $C_{c}(v)=s\left( \pm \beta_{s t s}\right)= \pm \beta_{t}$.

Proof of Proposition 5.2. Let $a_{1} \cdots a_{k}$ be the $\left(s_{1} \cdots s_{n}\right)$-sorting word for $v$ and let $s=s_{1}$. We split into several cases.

Case 1: $v \nsupseteq s$. Then $v \in W_{\langle s\rangle}$ and $C_{c}(v)=C_{s c}(v) \cup\left\{\alpha_{s}\right\}$, so $A_{c}(v)=A_{s c}(v)$. By induction on rank, $A_{s c}(v)=\left\{-\beta_{t}: t \in \operatorname{cov}(v)\right\}$.

Subcase 2a: $v \geq s$ and $s$ is a cover reflection of $v$. In this case $\operatorname{cov}(s v)$ is equal to $\{s t s: t \in(\operatorname{cov}(v) \backslash\{s\})\}$. We have $A_{\text {scs }}(s v)=\left\{-\beta_{\text {sts }}: t \in(\operatorname{cov}(v) \backslash\{s\})\right\}$ by induction on length. Because $C_{c}(v)=s C_{s c s}(s v)$ and since only $\pm \alpha_{s}$ have their 
positive/negative status changed by the action of the reflection $s$, either $A_{c}(v)=$ $s A_{s c s}(s v)$ or $A_{c}(v)=\left\{-\alpha_{s}\right\} \cup s A_{s c s}(s v)$. To finish the proof in this case, it remains to show that the latter case holds, or in other words that $\alpha_{s} \in C_{s c s}(s v)$. By Proposition 5.1, this is equivalent to showing that there is an unforced skip of some $r$ in the $i^{\text {th }}$ position of $a_{2} \cdots a_{k}$ with $s=a_{2} \cdots a_{i} r a_{i} \cdots a_{2}$.

Consider the $\left(s_{2} \cdots s_{n} s_{1}\right)$-sorting word $a_{1}^{\prime} \cdots a_{k}^{\prime}$ for $v$. Take $i$ to be the index such that $s=a_{1}^{\prime} \cdots a_{i}^{\prime} \cdots a_{1}^{\prime}$. Since $s$ is a cover reflection of $v, \operatorname{inv}(v)=\{s\} \cup \operatorname{inv}(s v)$. Thus $a_{1}^{\prime}, \ldots, a_{i-1}^{\prime}$ are the first $i-1$ letters of the $\left(s_{2} \cdots s_{n} s_{1}\right)$-sorting word for $s v$. By the uniqueness of sorting words, the words $a_{1}^{\prime}, \ldots, a_{i-1}^{\prime}$ and $a_{2}, \ldots, a_{i}$ are equal. Since $s$ is not an inversion of $s v, a_{i}^{\prime}$ is skipped at position $i$ in $a_{2} \cdots a_{k}$. This is an unforced skip, since $a_{1}^{\prime} \cdots a_{i}^{\prime}=a_{2} \cdots a_{i} a_{i}^{\prime}$ is reduced. This completes the proof in the case where $v \geq s$ and $s$ is a cover reflection of $v$.

Subcase 2b: $v \geq s$ and $s$ is not a cover reflection of $v$. Then

$$
\operatorname{cov}(s v)=\{s t s: t \in \operatorname{cov}(v)\} .
$$

By induction on length, $A_{s c s}(s v)=\left\{-\beta_{s t s}: t \in \operatorname{cov}(v)\right\}$. In this case we want to show that $A_{c}=s A_{s c s}(s v)$, or equivalently that $\alpha_{s} \notin C_{s c s}(s v)$. Appealing again to Proposition 5.1, we complete the proof by showing that there is no unforced skip in $a_{2} \cdots a_{k}$ whose corresponding reflection is $s$. Suppose to the contrary that there is an unforced skip of some $r$ in the $i^{\text {th }}$ position of $a_{2} \cdots a_{k}$ with $s=a_{2} \cdots a_{i} r a_{i} \cdots a_{2}$. Define $t_{j}^{\prime}=a_{2} \cdots a_{j} \cdots a_{2}$ for every $j$ with $2 \leq j \leq k$. By Lemma 5.8, $\omega_{s c s}\left(\alpha_{s}, \beta_{t_{j}^{\prime}}\right) \geq 0$ for every $j$ between $i+1$ and $k$ (inclusive), with strict inequality unless $s$ and $t_{j}^{\prime}$ commute. But, by Lemma 3.10, $\omega_{s c s}\left(\alpha_{s}, \beta_{t}\right) \leq 0$ for every reflection $t$, because $s$ is final in scs. Thus $s$ and $t_{j}^{\prime}$ commute for every $j$ with $i<j \leq k$. For each such $j$, let $t_{j}^{\prime \prime}=a_{i+1} \cdots a_{j} \cdots a_{i+1}$. Since $s=a_{2} \cdots a_{i} r a_{i} \cdots a_{2}$ commutes with $t_{j}^{\prime}=a_{2} \cdots a_{i} t_{j}^{\prime \prime} a_{i} \cdots a_{2}$, the reflection $r$ commutes with $t_{j}^{\prime \prime}$. Since the $t_{j}^{\prime \prime}$ 's are the inversions of $a_{i+1} \cdots a_{k}$, by Lemma 2.1, $r$ commutes with $a_{i+1} \cdots a_{k}$. So we have $v=a_{1} a_{2} \cdots a_{i} a_{i+1} \cdots a_{k}=a_{2} \cdots a_{i} r a_{i+1} \cdots a_{k}=a_{2} \cdots a_{i} a_{i+1} \cdots a_{k} r$. Since $\ell(v)=k$, the word $a_{2} \cdots a_{i} a_{i+1} \cdots a_{k} r$ is reduced. So $a_{2} \cdots a_{k} r=v$ covers $a_{2} \cdots a_{k}=s v$, contradicting the assumption that $s$ is not a cover reflection of $v$.

We now proceed to prove Propositions 5.3 and 5.4 again starting with preliminary lemmas. The second of the two lemmas is essentially a converse to Lemma 5.8.

Lemma 5.9. Let $v$ be c-sortable, with $c=s_{1} s_{2} \cdots s_{n}$. Let $\Omega_{c}(v)$ be the subword of $\left(s_{1} s_{2} \cdots s_{n}\right)^{\infty}$ which is the complement of the $\left(s_{1} s_{2} \cdots s_{n}\right)$-sorting word for $v$. Let $\left(r_{1}, r_{2}, \ldots, r_{n}\right)$ be the simple reflections, ordered according to when they first appear in $\Omega_{c}(v)$. Set $\beta_{i}=C_{c}^{r_{i}}(v)$.

With the above definitions, $E_{c}\left(\beta_{i}, \beta_{j}\right)=0$ for $i<j$.

Proof. We use the usual argument by induction on length and rank. The base case, where $v=e$, is immediate from the definition of $E_{c}$.

First, suppose that $v \geq s$. In particular, the orderings on $S$ obtained from $\Omega_{c}(v)$ and from $\Omega_{s c s}(s v)$ are the same. Now $E_{c}\left(\beta_{i}, \beta_{j}\right)=E_{c}\left(s C_{s c s}^{r_{i}}(s v), s C_{s c s}^{r_{j}}(s v)\right)$. By Lemma 3.3 the latter equals $E_{s c s}\left(C_{s c s}^{r_{i}}(s v), C_{s c s}^{r_{j}}(s v)\right)$, which by induction equals zero for $i<j$.

Now, suppose that $v \nsupseteq s$, so $v \in W_{\langle s\rangle}$. Then $s=r_{1}$ and $\beta_{1}=\alpha_{s}$. For $i>1$, the $\operatorname{root} \beta_{i}$ lies in $\Phi_{\langle s\rangle}$ and, thus, $E_{c}\left(\beta_{1}, \beta_{i}\right)=0$. The ordering on $S \backslash\{s\}$ obtained from $\Omega_{s c}(v)$ is the restriction of the ordering obtained from $\Omega_{c}(v)$. Thus if $1<i<j$, then $E_{c}\left(\beta_{i}, \beta_{j}\right)=0$ by induction on rank. 
Lemma 5.10. Let $t_{1}, t_{2}, \ldots, t_{j}, t, t_{j+1}, \ldots, t_{N}$ be a sequence of $N+1$ distinct reflections of $W$, and let $v$ be a c-sortable element of $W$. Suppose that the following conditions hold:

(i) $t_{1}, t_{2}, \ldots, t_{j}, t_{j+1}, \ldots, t_{N}$ is the reflection sequence of a $c$-sorting word for $v$.

(ii) $t_{1}, t_{2}, \ldots, t_{j}, t$ is the reflection sequence of a reduced word.

(iii) $\omega_{c}\left(\beta_{t_{i}}, \beta_{t}\right) \geq 0$ for all $i \leq j$, with strict inequality unless $t_{i}$ and $t$ commute, and

(iv) $\omega_{c}\left(\beta_{t}, \beta_{t_{k}}\right) \geq 0$ for all $k>j$, with strict inequality unless $t$ and $t_{k}$ commute.

Then $t$ is an unforced skip of $v$.

Proof. Let $s$ be initial in $c$. If $v \nsupseteq s$, then $v \in W_{\langle s\rangle}$ and each $t_{i} \in W_{\langle s\rangle}$. Let $v^{\prime}$ be the element with a reduced word having reflection sequence $t_{1}, \ldots, t_{j}, t$. By Proposition 3.11, applied twice, we see that $v^{\prime}$ is $c$-sortable. We claim that either $t=s$ or $t \in W_{\langle s\rangle}$. If $t \notin W_{\langle s\rangle}$, then we have $v^{\prime} \notin W_{\langle s\rangle}$, so, since $v^{\prime}$ is $c$-sortable, $s \leq v^{\prime}$. But $t$ is the only inversion of $v^{\prime}$ that is not an inversion of $v$, and $s \not \leq v$. Thus $t=s$, and this proves the claim. Since $v \nsupseteq s$ and $s$ is initial in $c$, we have $s \in \operatorname{ufs}_{c}(v)$. Thus, by the claim, it remains only to consider the case $t \in W_{\langle s\rangle}$. By Lemma 3.7 we see that (iii) and (iv) also hold with respect to $\omega_{s c}$. Thus by induction on rank, $t \in \mathrm{ufs}_{s c}(v) \subset \operatorname{ufs}_{c}(v)$.

If $v \geq s$, then $s \in\left\{t_{1}, \ldots, t_{N}\right\}$, so $t \neq s$. Since $s$ is initial in $c$, we can transpose initial commuting entries in the sequence $t_{1}, \ldots, t_{N}$ to make $s$ appear first in the sequence, without upsetting properties (i)-(iv). By Lemma 3.8 and Proposition 2.29, the sequence $s t_{2} s, s t_{3} s, \ldots, s t_{j} s, s t s, s t_{j+1} s, \ldots, s t_{N} s$ satisfies (i)-(iv) with respect to $s c s$. By induction on $N$ we have $s t s \in \mathrm{ufs}_{s c s}(s v)$, so $t \in \operatorname{ufs}_{c}(v)$.

We will soon turn to the proofs of Propositions 5.3 and 5.4. We will deduce both these propositions from the following result.

Proposition 5.11. Let $s$ be either initial or final in $c$. Let $v$ be c-sortable with $s \in \operatorname{cov}(v)$ and let $t$ be a reflection with $\pm \beta_{t}$ in $C_{c}(v) \backslash\left\{-\alpha_{s}\right\}$.

(i) If $s$ is final in $c$ or $t \in \operatorname{cov}(v)$, then $t \in W_{\langle s\rangle}$.

(ii) If $s$ is initial in $c$ and $t \in \mathrm{ufs}_{c}(v)$, then sts $\in W_{\langle s\rangle}$.

By Proposition 5.1, either $t \in \operatorname{cov}(v)$ or $t \in \mathrm{ufs}_{c}(v)$, so one of these cases applies.

Proof. Proposition 5.2 and the fact that $s$ is a cover reflection of $v$ imply that $-\alpha_{s} \in C_{c}(v)$. By Lemma [5.9, either $E_{c}\left(-\alpha_{s}, \pm \beta_{t}\right)=0$ or $E_{c}\left( \pm \beta_{t},-\alpha_{s}\right)=0$. If $E_{c}\left(-\alpha_{s}, \pm \beta_{t}\right)=0$ and $s$ is initial in $c$, then $t$ is in $W_{\langle s\rangle}$ by Lemma 3.1. Similarly, if $E_{c}\left( \pm \beta_{t},-\alpha_{s}\right)=0$ and $s$ is final in $c$, then $t$ is in $W_{\langle s\rangle}$ by Lemma 3.2. If $E_{c}\left( \pm \beta_{t},-\alpha_{s}\right)=0$ and $s$ is initial in $c$, then by Lemma 3.3.

$$
E_{s c s}\left( \pm \beta_{s t s},-\alpha_{s}\right)=E_{s c s}\left( \pm s \beta_{t}, s \alpha_{s}\right)=E_{c}\left( \pm \beta_{t}, \alpha_{s}\right)=0,
$$

so $s t s \in W_{\langle s\rangle}$ by Lemma 3.2. Similarly, if $E_{c}\left(-\alpha_{s}, \pm \beta_{t}\right)=0$ and $s$ is final in $c$, then $s t s \in W_{\langle s\rangle}$. We have shown that either $t$ or $s t s$ is in $W_{\langle s\rangle}$. It remains to establish the more specific claims of (i) and (ii). If $s$ and $t$ commute, then $t=s t s$ and both claims follow immediately, so we may assume that they do not commute. 
Consider the generalized rank two parabolic subgroup $W^{\prime}$ of $W$ which contains $s$ and $t$. Since $s$ and $t$ do not commute, $W^{\prime}$ is not commutative. By Lemmas 2.14 and 2.16. $s$ is a canonical generator of $W^{\prime}$ and the other canonical generator is in $W_{\langle s\rangle}$. Let $p$ be this other generator. We break into cases:

Case 1: $t$ is a cover reflection of $v$. Then $t \in \operatorname{inv}(v)$. Since we assumed that $s$ is also a cover reflection of $v$, we have $\operatorname{inv}(v)=\operatorname{inv}(s v) \backslash\{s\}$, and thus sts is also an inversion of $v$. Since either $t$ or sts is $p$, we know that $s$ and $p$ are both inversions of $v$, and thus every reflection of $W^{\prime}$ is an inversion of $v$ by Lemma 2.17 Then, by Lemma 2.26, $t$ must be $p$. In particular, $t$ is in $W_{\langle s\rangle}$, as desired.

Case 2: $t$ is an unforced skip of $v$. In this case, let $a_{1} a_{2} \ldots a_{k}$ be a $c$-sorting word for $v$ and let $t=a_{1} a_{2} \cdots a_{i} r a_{i} \cdots a_{2} a_{1}$ for an unforced skip of $r$ at position $(i+1)$ in $v$. Let $v^{\prime}$ be the $c$-sortable element $a_{1} a_{2} \cdots a_{i} r$. Now, since $s$ is a cover reflection of $v$, we have $\operatorname{inv}(v)=s \operatorname{inv}(v) s$. Using the fact that $t$ is not an inversion of $v$, we deduce from Lemma 2.17 that $\operatorname{inv}(v) \cap W^{\prime}=\{s\}$. In particular, $p$ and sps are not inversions of $v$. Now, $\operatorname{inv}\left(v^{\prime}\right) \backslash\{t\} \subseteq \operatorname{inv}(v)$, so $\left(\operatorname{inv}\left(v^{\prime}\right) \cap W^{\prime}\right) \backslash\{t\}$ is either empty or $\{s\}$. Applying Lemma 2.17 either $\left(\operatorname{inv}\left(v^{\prime}\right) \cap W^{\prime}\right)=\{p\}=\{t\}$ or $\left(\operatorname{inv}\left(v^{\prime}\right) \cap W^{\prime}\right)=\{s, s p s\}=\{s, t\}$. If $s$ is initial in $c$, then $a_{1}=s$ so $s \in \operatorname{inv}\left(v^{\prime}\right)$, so $\left(\operatorname{inv}\left(v^{\prime}\right) \cap W^{\prime}\right)=\{s$, sps $\}=\{s, t\}$, and thus sps $=t$, so that sts $=p \in W_{\langle s\rangle}$. If $s$ is final in $c$, then, since $v^{\prime}$ is $c$-aligned with respect to $W^{\prime},\left(\operatorname{inv}\left(v^{\prime}\right) \cap W^{\prime}\right)$ cannot be $\{s, s p s\}$. (Here we use the fact that $W^{\prime}$ is not commutative.) Thus $\left(\operatorname{inv}\left(v^{\prime}\right) \cap W^{\prime}\right)=\{p\}=\{t\}$. Now $t=p \in W_{\langle s\rangle}$.

We now prove Propositions 5.3 and 5.4

Proof of Proposition 5.3. The assertion that $s \in \operatorname{cov}(v)$ is Lemma 3.12, so by Proposition 5.11 we deduce that $t$ is in $W_{\langle s\rangle}$ whenever $\pm \beta_{t} \in C_{c}(v) \backslash\left\{-\alpha_{s}\right\}$. Thus $\operatorname{cov}(v) \backslash\{s\} \subseteq W_{\langle s\rangle}$ by Lemma [5.2. Assertion (i) now holds by Lemma 2.22, and assertion (ii) then follows by Lemma 2.23

Since there are exactly $n$ reflections in $\operatorname{cov}(v) \cup \operatorname{ufs}_{c}(v)$ and exactly $n-1$ reflections in $\operatorname{cov}\left(v_{\langle s\rangle}\right) \cup \operatorname{ufs}_{c s}\left(v_{\langle s\rangle}\right)$, assertion (ii) implies that the sets $\operatorname{ufs}_{c}(v)$ and $\operatorname{ufs}_{c s}\left(v_{\langle s\rangle}\right)$ have the same size. Thus to prove assertion (iii), it is enough to show that $\mathrm{ufs}_{c}(v) \subseteq$ $\operatorname{ufs}_{c s}\left(v_{\langle s\rangle}\right)$. Suppose $t \in \operatorname{ufs}_{c}(v)$ and let $a_{1} \cdots a_{\ell(v)}$ be a $c$-sorting word for $v$ with associated reflection sequence $t_{1}, \ldots, t_{\ell(v)}$. Let $t=a_{1} \cdots a_{j} r a_{j} \cdots a_{1}$ for a skip of $r$ in the $(j+1)^{\text {st }}$ position. By Lemmas [5.6 and 5.8 and Proposition 3.11, the sequence $t_{1}, t_{2}, \ldots, t_{j}, t, t_{j+1}, \ldots, t_{\ell(v)}$ satisfies the hypotheses of Lemma 5.10. Let $t_{1}^{\prime}, t_{2}^{\prime}, \ldots, t_{j^{\prime}}^{\prime}, t, t_{j^{\prime}+1}^{\prime}, \ldots, t_{\ell\left(v_{\langle s\rangle}\right)}^{\prime}$ be the subsequence of $t_{1}, t_{2}, \ldots, t_{j}, t, t_{j+1}$, $\ldots, t_{\ell(v)}$ consisting of those reflections in the larger sequence which lie in $W_{\langle s\rangle}$. By Proposition 5.11 $t$ is in this subsequence.

Arguing as in the proof of Proposition 3.13, we see that $t_{1}^{\prime}, t_{2}^{\prime}, \ldots, t_{\ell\left(v_{\langle s\rangle}\right)}^{\prime}$ is the reflection sequence of a reduced word $a_{1}^{\prime} \ldots a_{\ell\left(v_{\langle s\rangle}\right)}^{\prime}$ for $v_{\langle s\rangle}$. Furthermore, $a_{1}^{\prime} \cdots a_{\ell\left(v_{\langle s\rangle}\right)}^{\prime}$ can be converted, by a sequence of transpositions of adjacent commuting letters, to a $c s$-sorting word for $v_{\langle s\rangle}$. The sequence $t_{1}, t_{2}, \ldots, t_{j}, t, t_{j+1}, \ldots, t_{\ell(v)}$ satisfies the hypotheses of Lemma 5.10, so Lemmas 2.16, 2.17 and 3.7 imply that $t_{1}^{\prime}, \ldots, t_{j^{\prime}}^{\prime}, t$, $t_{j^{\prime}+1}^{\prime}, \ldots, t_{\ell\left(v_{\langle s\rangle}\right)}^{\prime}$ satisfies the hypotheses of Lemma [5.10. Thus $t \in \mathrm{ufs}_{c s}\left(v_{\langle s\rangle}\right)$ by Lemma 5.10

Proof of Proposition 5.4. By hypothesis, $s \in \operatorname{cov}(v)$, so Proposition 5.11 applies. As in the previous proof, every cover reflection of $v$, besides $s$, is in $W_{\langle s\rangle}$. Now (i) follows by Lemma 2.22 and (ii) follows from (i) by Lemma 2.23 
We now prove assertion (iii). As in the proof of Proposition 5.3 it is enough to show that ufs $\operatorname{uf}_{c}(v) \subseteq\left\{s t s: t \in \mathrm{ufs}_{s c}\left(v_{\langle s\rangle}\right)\right\}$. Let $t \in \mathrm{ufs}_{c}(v)$. Then sts $\in \mathrm{ufs}_{s c s}(s v)$. Since $s t s \in W_{\langle s\rangle}$, we argue, exactly as in the last paragraph of the proof of Proposition 5.3 that sts $\in \mathrm{ufs}_{s c}\left((s v)_{\langle s\rangle}\right)$.

Now, $\operatorname{inv}\left((s v)_{\langle s\rangle}\right)=\operatorname{inv}(s v) \cap W_{\langle s\rangle}$. Since $s$ is a cover reflection of $v$, we have $\operatorname{inv}(s v)=\operatorname{inv}(v) \backslash\{s\}$, and thus $\operatorname{inv}(s v) \cap W_{\langle s\rangle}=\operatorname{inv}(v) \cap W_{\langle s\rangle}=\operatorname{inv}\left(v_{\langle s\rangle}\right)$. Thus $(s v)_{\langle s\rangle}$ and $v_{\langle s\rangle}$ are equal because $\operatorname{inv}\left((s v)_{\langle s\rangle}\right)=\operatorname{inv}\left(v_{\langle s\rangle}\right)$. So $s t s \in \mathrm{ufs}_{s c}\left(v_{\langle s\rangle}\right)$, or in other words $t \in\left\{s t s: t \in \mathrm{ufs}_{s c}\left(v_{\langle s\rangle}\right)\right\}$.

\section{The PROJECTION $\pi_{\downarrow}^{c}$}

In this section we define a downward projection $\pi_{\downarrow}^{c}$ from $W$ to the $c$-sortable elements of $W$. Let $c$ be a Coxeter element of $W$ and let $s$ be initial in $c$. Then, for each $w \in W$, let

$$
\pi_{\downarrow}^{c}(w)= \begin{cases}s \pi_{\downarrow}^{s c s}(s w) & \text { if } w \geq s, \\ \pi_{\downarrow}^{s c}\left(w_{\langle s\rangle}\right) & \text { if } w \nsupseteq s .\end{cases}
$$

This definition is recursive, by the usual induction on length and rank. As a base for the recursion, set $\pi_{\downarrow}^{c}(e)=e$ for any $c$ in any $W$. Recall that by convention, the map $\pi_{\downarrow}^{s c}$ is a map defined on the parabolic subgroup $W_{\langle s\rangle}$.

After establishing that $\pi_{\downarrow}^{c}$ is well-defined and presenting its most basic properties, we prove two major theorems. First:

Theorem 6.1. $\pi_{\downarrow}^{c}$ is order preserving.

As an immediate consequence of Theorem 6.1 and the basic properties of $\pi_{\downarrow}^{c}$, we have the following corollary, which makes the connection between the description of $\pi_{\downarrow}^{c}$ in Section 1.1 and the recursive definition above.

Corollary 6.2. Let $w \in W$. Then $\pi_{\downarrow}^{c}(w)$ is the unique maximal c-sortable element below $w$ in the weak order.

The other main result of this section is Theorem 6.3. which gives a geometric description of the fibers of $\pi_{\downarrow}^{c}$. Define a cone

$$
\operatorname{Cone}_{c}(v)=\bigcap_{\beta \in C_{c}(v)}\left\{x^{*} \in V^{*}:\left\langle x^{*}, \beta\right\rangle \geq 0\right\}
$$

and recall that $D$ is the cone $\bigcap_{s \in S}\left\{x^{*} \in V^{*}:\left\langle x^{*}, \alpha_{s}\right\rangle \geq 0\right\}$. The definition of Cone $_{c}(v)$, like the definition of $D$, depends on the choice of symmetrizable Cartan matrix $A$ for $W$. Since each $C_{c}(v)$ is a basis for $V$, each cone Cone $_{c}(v)$ is a fulldimensional simplicial cone.

Theorem 6.3. Let $v$ be c-sortable. Then $\pi_{\downarrow}^{c}(w)=v$ if and only if $w D \subseteq \operatorname{Cone}_{c}(v)$.

The condition $w D \subseteq \operatorname{Cone}_{c}(v)$ is equivalent to the following: $t \in \operatorname{inv}(w)$ for every $-\beta_{t} \in A_{c}(v)$, and $t \notin \operatorname{inv}(w)$ for every $\beta_{t} \in B_{c}(v)$. Less formally (and as an explanation of the use of the symbols $A$ and $B), \pi_{\downarrow}^{c}(w)$ is $v$ if and only if $w D$ is above the hyperplane orthogonal to $\beta$ for every $\beta \in A_{c}(v)$ and below the hyperplane orthogonal to $\beta$ for every $\beta \in B_{c}(v)$. Propositions 5.1 and 5.2 allow Theorem 6.3 to be restated as follows. 
Theorem 6.4. Let $w \in W$ and let $v$ be c-sortable. Then $\pi_{\downarrow}^{c}(w)=v$ if and only if both of the following two conditions hold:

(i) $\operatorname{cov}(v) \subseteq \operatorname{inv}(w)$ and

(ii) $\operatorname{ufs}_{c}(v) \cap \operatorname{inv}(w)=\emptyset$.

Example 6.5. The computations of Example 5.5 show that, in the notation of that example, $\pi_{\downarrow}^{c}(w)=v$ if and only if $q$ and pqrqp are inversions of $w$ and pqrpqprqp is not. See the discussion of Figures 3 and 4 for more examples and for a geometric view of Theorem 6.3

We now establish the basic properties of $\pi_{\downarrow}^{c}$, beginning with well-definition. Calculating $\pi_{\downarrow}^{c}$ recursively involves the choice of a sequence of simple generators. $A$ priori it appears that the result might depend on the choice of this sequence. In fact, the result is independent of the sequence chosen:

Lemma 6.6. For any $w \in W$, the element $\pi_{\downarrow}^{c}(w)$ is independent of which choice is made of an initial element in each step of the recursion.

Proof. If $c$ has only one initial element, then the result follows by induction on length or rank. Otherwise, let $s$ and $s^{\prime}$ be two distinct initial elements of $c$, so that in particular $s$ and $s^{\prime}$ commute. The proof breaks into four cases according to whether $s$ and $s^{\prime}$ are below $w$ in weak order. In each case we use $s$ and $s^{\prime}$ to calculate $\pi_{\downarrow}^{c}(w)$ in two ways. Each of these involves an expression which, by induction on length or rank, is independent of the subsequent choices of initial letters. Thus in every case, it is sufficient to show: First, that $\pi_{\downarrow}^{c}(w)$ can be evaluated by choosing $s$ and then $s^{\prime}$ or by choosing $s^{\prime}$ and then $s$; and second, that the two expressions for $\pi_{\downarrow}^{c}(w)$ obtained in this manner coincide.

If $w \nsupseteq s$ and $w \nsupseteq s^{\prime}$, then the two possibilities for $\pi_{\downarrow}^{c}(w)$ are $\pi_{\downarrow}^{s c}\left(w_{\langle s\rangle}\right)$ and $\pi_{\downarrow}^{s^{\prime} c}\left(w_{\left\langle s^{\prime}\right\rangle}\right)$. Since $s$ and $s^{\prime}$ commute, $s^{\prime}$ is initial in $s c$ and $s$ is initial in $s^{\prime} c$. Since $w_{\langle s\rangle} \leq w$ we have $w_{\langle s\rangle} \nsucceq s^{\prime}$, and similarly $w_{\left\langle s^{\prime}\right\rangle} \nsupseteq s$. Thus $\pi_{\downarrow}^{s c}\left(w_{\langle s\rangle}\right)=$ $\pi_{\downarrow}^{s^{\prime} s c}\left(w_{\left\langle s, s^{\prime}\right\rangle}\right)=\pi_{\downarrow}^{s^{\prime} c}\left(w_{\left\langle s^{\prime}\right\rangle}\right)$. (Here the notation $\left\langle s, s^{\prime}\right\rangle$ stands for $S \backslash\left\{s, s^{\prime}\right\}$.)

If $w$ is above exactly one of $s$ and $s^{\prime}$, then we may as well take $w \geq s$ and $w \geq s^{\prime}$. The two possibilities for $\pi_{\downarrow}^{c}(w)$ are $s \pi_{\downarrow}^{s c s}(s w)$ and $\pi_{\downarrow}^{s^{\prime} c}\left(w_{\left\langle s^{\prime}\right\rangle}\right)$. Again, since $s$ and $s^{\prime}$ commute, $s^{\prime}$ is initial in scs and $s$ is initial in $s^{\prime} c$. Since the map $x \mapsto x_{\left\langle s^{\prime}\right\rangle}$ is order preserving and $s_{\left\langle s^{\prime}\right\rangle}=s$, we have $w_{\left\langle s^{\prime}\right\rangle} \geq s$. Since $\operatorname{inv}(s w)=s(\operatorname{inv}(w)) s \backslash\{s\}$ and $s s^{\prime} s=s^{\prime}$, the fact that $w \nsupseteq s^{\prime}$ implies that $s w \geq s^{\prime}$. Thus $\pi_{\downarrow}^{s c s}(s w)=$ $\pi_{\downarrow}^{s^{\prime}} s c s\left((s w)_{\left\langle s^{\prime}\right\rangle}\right)$ and $\pi_{\downarrow}^{s^{\prime} c}\left(w_{\left\langle s^{\prime}\right\rangle}\right)=s \pi_{\downarrow}^{s s^{\prime} c s}\left(s\left(w_{\left\langle s^{\prime}\right\rangle}\right)\right)$. We have $s^{\prime} s c s=s s^{\prime} c s$, and we compare inversion sets to see that $(s w)_{\left\langle s^{\prime}\right\rangle}=s\left(w_{\left\langle s^{\prime}\right\rangle}\right)$. Specifically,

$$
\operatorname{inv}\left((s w)_{\left\langle s^{\prime}\right\rangle}\right)=\operatorname{inv}(s w) \cap W_{\left\langle s^{\prime}\right\rangle}=[s(\operatorname{inv}(w)) s \backslash\{s\}] \cap W_{\left\langle s^{\prime}\right\rangle},
$$

which is equal to

$$
\left[s(\operatorname{inv}(w)) s \cap W_{\left\langle s^{\prime}\right\rangle}\right] \backslash\{s\}=\left[s\left(\operatorname{inv}(w) \cap W_{\left\langle s^{\prime}\right\rangle}\right) s\right] \backslash\{s\}=\operatorname{inv}\left(s\left(w_{\left\langle s^{\prime}\right\rangle}\right)\right) .
$$

If $w \geq s$ and $w \geq s^{\prime}$, then the two possibilities for $\pi_{\downarrow}^{c}(w)$ are $s \pi_{\downarrow}^{s c s}(s w)$ and $s^{\prime} \pi_{\downarrow}^{s^{\prime} c s^{\prime}}\left(s^{\prime} w\right)$. Again, $s^{\prime}$ is initial in $s c s, s$ is initial in $s^{\prime} c s^{\prime}, s^{\prime} w \geq s$ and $s w \geq s^{\prime}$. Thus the two expressions for $\pi_{\downarrow}^{c}(w)$ are $s^{\prime} s \pi_{\downarrow}^{s^{\prime}} s c s s^{\prime}\left(s^{\prime} s w\right)$ and $s s^{\prime} \pi_{\downarrow}^{s s^{\prime} c s^{\prime} s}\left(s s^{\prime} w\right)$, which are equal because $s$ and $s^{\prime}$ commute.

We note the following consequences of the definition of $\pi_{\downarrow}^{c}$. 
Proposition 6.7. The element $\pi_{\downarrow}^{c}(w)$ is c-sortable for any $w \in W$. We have $\pi_{\downarrow}^{c}(w) \leq w$, with equality if and only if $w$ is c-sortable.

Proof. Let $s$ be initial in $c$. We again argue by induction on length and rank. If $w \geq s$, then $\pi_{\downarrow}^{c}(w)=s \pi_{\downarrow}^{s c s}(s w)$. By induction on length, $\pi_{\downarrow}^{s c s}(s w) \leq s w$, with equality if and only if $s w$ is $s c s$-sortable. Now Propositions 2.18 and 2.29 imply that $\pi_{\downarrow}^{c}(w) \leq w$, with equality if and only if $w$ is $c$-sortable. Also, by induction on length, $\pi_{\downarrow}^{s c s}(s w)$ is $s c s$-sortable, so, by Proposition 2.29, $s \pi_{\downarrow}^{s c s}(s w)=\pi_{\downarrow}^{c}(w)$ is $c$-sortable.

If $w \nsupseteq s$, then $\pi_{\downarrow}^{c}(w)=\pi_{\downarrow}^{s c}\left(w_{\langle s\rangle}\right)$, and by induction on rank, $\pi_{\downarrow}^{s c}\left(w_{\langle s\rangle}\right) \leq w_{\langle s\rangle}$ with equality if and only if $w_{\langle s\rangle}$ is $s c$-sortable. Since $w_{\langle s\rangle} \leq w$ with equality if and only if $w \in W_{\langle s\rangle}$, Proposition 2.29 shows that $\pi_{\downarrow}^{c}(w) \leq w$, with equality as desired. Also, $\pi_{\downarrow}^{c}(w)=\pi_{\downarrow}^{s c}\left(w_{\langle s\rangle}\right)$, which is, by induction, $s c$-sortable and thus $c$-sortable.

Proposition 6.8. The map $\pi_{\downarrow}^{c}$ is idempotent, i.e. $\pi_{\downarrow}^{c} \circ \pi_{\downarrow}^{c}=\pi_{\downarrow}^{c}$.

Proof. Let $w \in W$. Then $\pi_{\downarrow}^{c}(w)$ is $c$-sortable by Proposition 6.7 Thus by Proposition 6.7 again, $\pi_{\downarrow}^{c}\left(\pi_{\downarrow}^{c}(w)\right)=\pi_{\downarrow}^{c}(w)$.

Proposition 6.9. Let $s$ be initial in $c$. Then $w \geq s$ if and only if $\pi_{\downarrow}^{c}(w) \geq s$.

This is actually true without the hypothesis that $s$ is initial, as an immediate corollary of Theorem 6.1. However, the proof of Theorem 6.1 is much harder and explicitly depends on Proposition 6.9.

Proof. If $w \nsupseteq s$, then Proposition 6.7 implies that $\pi_{\downarrow}^{c}(w) \nsupseteq s$. If $w \geq s$, Proposition 2.18 states that $s w \geq s$. Then $\pi_{\downarrow}^{s c s}(s w) \nsupseteq s$ by Proposition 6.7. Applying Proposition 2.18 again, we obtain $\pi_{\downarrow}^{c}(w)=s \pi_{\downarrow}^{s c s}(s w) \geq s$.

The map $\pi_{\downarrow}^{c}$ is also well-behaved with respect to standard parabolic subgroups. A very strong statement about $\pi_{\downarrow}^{c}$ and standard parabolic subgroups occurs later as Proposition 6.13. Here is a much simpler result.

Proposition 6.10. Let $c$ be a Coxeter element of $W$, let $J \subseteq S$ and let $c^{\prime}$ be the restriction of $c$ to $W_{J}$. If $w \in W_{J}$, then $\pi_{\downarrow}^{c}(w)=\pi_{\downarrow}^{c^{\prime}}(w)$.

Note that the left hand side of this equation involves a map $\pi_{\downarrow}^{c}$ defined on $W$, whereas the right hand side uses a map $\pi_{\downarrow}^{c^{\prime}}$ defined on $W_{J}$.

Proof. It is enough to prove the proposition in the case where $J=S \backslash\{s\}$ for some $s \in S$.

Let $s^{\prime}$ be initial in $c$. We argue as usual by induction on the length of $w$ and on the rank of $W$. If $s^{\prime}=s$, then the assertion of the proposition is that $\pi_{\downarrow}^{c}(w)=$ $\pi_{\downarrow}^{s c}(w)$, which is true by definition because $w=w_{\langle s\rangle} ¥ s$. If $s^{\prime} \neq s$, then consider two cases, $w \geq s^{\prime}$ and $w \geq s^{\prime}$. In the case $w \geq s^{\prime}$, the element $s^{\prime} w$ is in $W_{\langle s\rangle}$ and $s^{\prime} c^{\prime} s^{\prime}$ is the Coxeter element of $W_{\langle s\rangle}$ obtained by deleting $s$ from $s^{\prime} c s^{\prime}$. Thus induction on length shows that $\pi_{\downarrow}^{s^{\prime} c s^{\prime}}\left(s^{\prime} w\right)=\pi_{\downarrow}^{s^{\prime} c^{\prime} s^{\prime}}\left(s^{\prime} w\right)$, so that $\pi_{\downarrow}^{c}(w)=\pi_{\downarrow}^{c^{\prime}}(w)$. In the case $w \nsupseteq s^{\prime}$ we have $\pi_{\downarrow}^{c}(w)=\pi_{\downarrow}^{s^{\prime} c}\left(w_{\left\langle s^{\prime}\right\rangle}\right)$, which by induction on rank equals $\pi_{\downarrow}^{s^{\prime} c^{\prime}}\left(w_{\left\langle s^{\prime}\right\rangle}\right)=\pi_{\downarrow}^{c^{\prime}}(w)$.

We now proceed to prove Theorems 6.1 and 6.3 beginning with a special case of Theorem 6.3. This special case will be used to prove Theorem 6.1, which will in turn be used in the proof of the full version of Theorem 6.3 
Proposition 6.11. Let $v$ be c-sortable and let $v \leq w$. Then $\pi_{\downarrow}^{c}(w)=v$ if and only if $w D \subseteq$ Cone $_{c}(v)$.

Since $\pi_{\downarrow}^{c}(w)=v$ implies $w \geq v$, Proposition 6.11 falls short of Theorem 6.3 only in the following sense: The proposition guarantees that $w D \subseteq \operatorname{Cone}_{c}\left(\pi_{\downarrow}^{c}(w)\right)$, but a priori it is possible that there are additional sortable elements $v$ (not below $w$ ) such that $w D \subseteq \operatorname{Cone}_{c}(v)$.

Proof. Our proof is by induction on rank and on the length of $v$. The base for induction on rank is trivial, and the base for induction on length is the observation that $\operatorname{Cone}_{c}(e)=D$ when $e$ is the identity element of $W$. Let $s$ be initial in $c$. We again break into several cases.

Case 1: $w \nsupseteq s$ and $v \nsupseteq s$. Since $v$ is $c$-sortable and $v \nsupseteq s$, we have $v \in W_{\langle s\rangle}$. Furthermore, $C_{c}(v)=C_{s c}(v) \cup\left\{\alpha_{s}\right\}$, so that

$$
\begin{aligned}
\operatorname{Cone}_{c}(v) & =V_{\geq s}^{*} \cap \bigcap_{\beta \in C_{s c}(v)}\left\{x^{*} \in V_{\langle s\rangle}:\left\langle x^{*}, \beta\right\rangle \geq 0\right\} \\
& =V_{\geq s}^{*} \cap\left(P_{\langle s\rangle}\right)^{-1}\left(\operatorname{Cone}_{s c}(v)\right),
\end{aligned}
$$

where $V_{\ngtr s}^{*}=\left\{x^{*} \in V^{*}:\left\langle x^{*}, \alpha_{s}\right\rangle \geq 0\right\}$ and $P_{\langle s\rangle}$ is the projection defined in Section 2.4.

Suppose that $\pi_{\downarrow}^{c}(w)=v$, so $\pi_{\downarrow}^{s c}\left(w_{\langle s\rangle}\right)=v$. Since $w \geq v$, we have $w_{\langle s\rangle} \geq$ $v_{\langle s\rangle}=v$, so, by induction on rank, we deduce that $w_{\langle s\rangle} D_{\langle s\rangle} \subseteq \operatorname{Cone}_{s c}(v)$. Then $w D \subseteq P_{\langle s\rangle}^{-1}\left(w_{\langle s\rangle} D_{\langle s\rangle}\right) \subseteq P_{\langle s\rangle}^{-1}\left(\operatorname{Cone}_{s c}(v)\right)$. Also $w D \subseteq V_{\nsucceq s}^{*}$, because $w \nsupseteq s$. So $w D \subseteq P_{\langle s\rangle}^{-1}\left(\operatorname{Cone}_{s c}(v)\right) \cap V_{\Varangle s}^{*}=\operatorname{Cone}_{c}(v)$, as desired.

Conversely, suppose that $w D \subseteq \operatorname{Cone}_{c}(v)$, so $P_{\langle s\rangle}(w D) \subseteq \operatorname{Cone}_{s c}(v)$. Now, Cone $_{s c}(v)$ is a union of cones of the form $x D_{\langle s\rangle}$ for $x \in W_{\langle s\rangle}$. In particular, Cone $_{s c}(v)$ contains the unique cone $x D_{\langle s\rangle}$ in which $P_{\langle s\rangle}(w D)$ lies, namely the cone $w_{\langle s\rangle} D_{\langle s\rangle}$. Thus $w_{\langle s\rangle} D_{\langle s\rangle} \subseteq \mathrm{Cone}_{s c}(v)$ and, by induction on rank, $\pi_{\downarrow}^{s c}\left(w_{\langle s\rangle}\right)=v$. But $\pi_{\downarrow}^{c}(w)=\pi_{\downarrow}^{s c}\left(w_{\langle s\rangle}\right)$, so $\pi_{\downarrow}^{c}(w)=v$ as desired.

Case 2: $w \geq s$ and $v \geq s$. Since $v \geq s$, the equality $\pi_{\downarrow}^{c}(w)=v$ is equivalent to $\pi_{\downarrow}^{s c s}(s w)=s v$. We have $s v \leq s w$ by Proposition 2.18. Thus by induction on the length of $w$, the equality $\pi_{\downarrow}^{s c s}(s w)=s v$ is equivalent to $s w D \subseteq$ Cone $_{s c s}(s v)$ or, in other words, $w D \subset s \cdot \operatorname{Cone}_{s c s}(s v)=\operatorname{Cone}_{c}(v)$.

Case 3: $w \geq s$ and $v \nsupseteq s$. By Proposition 6.7 $\pi_{\downarrow}^{c}(w) \neq v$. By definition, $C_{c}(v)$ contains the element $\alpha_{s}$, but interior points $p$ of $w D$ have $\left\langle p, \alpha_{s}\right\rangle<0$, so that $w D \nsubseteq \operatorname{Cone}_{c}(v)$.

Case 4: $w \nsupseteq s$ and $v \geq s$. This is impossible by the hypothesis $v \leq w$.

Note that the only use of the hypothesis $v \leq w$ is to rule out Case 4 and to prove the inequalities (e.g. $s v \leq s w$ in Case 2) needed to invoke induction.

We are almost ready to prove Theorem 6.1. The inductive structure of the proof is complex, so we separate out the trickiest part as a lemma. For $W$ a Coxeter group, $c$ a Coxeter element of $W$ and $x$ and $y \in W$, let $\Sigma(W, c, x, y)$ denote the statement:

$$
\text { "If } y \geq x \text { then } \pi_{\downarrow}^{c}(y) \geq \pi_{\downarrow}^{c}(x) \text {." }
$$

Theorem 6.1 states that $\Sigma(W, c, x, y)$ is true for all $(W, c, x, y)$. 
Lemma 6.12. Let $W$ be a Coxeter group, $c$ a Coxeter element of $W, s \in S$ and $y \in W$. Suppose that both of the following conditions hold:

(i) If the rank of $\hat{W}$ is less than the rank of $W$, then $\Sigma(\hat{W}, \hat{c}, \hat{x}, \hat{y})$ is true for any Coxeter element $\hat{c}$ and any $\hat{x}, \hat{y} \in \hat{W}$.

(ii) $\Sigma(W, \hat{c}, \hat{x}, \hat{y})$ is true for any Coxeter element $\hat{c}$ and any $\hat{x}, \hat{y}$ with $\ell(\hat{y})<\ell(y)$. Then $\Sigma(W, c, s, y)$ is true.

Proof. Let $s^{\prime}$ be initial in $c$. If $y \geq s$, then the claim is trivial, so assume $y \geq s$. The case $s=s^{\prime}$ is Proposition 6.9, so we may also assume that $s \neq s^{\prime}$.

If $y \nsupseteq s^{\prime}$, then $\pi_{\downarrow}^{c}(y)=\pi_{\downarrow}^{s^{\prime} c}\left(y_{\left\langle s^{\prime}\right\rangle}\right)$. Since $y_{\left\langle s^{\prime}\right\rangle} \geq s_{\left\langle s^{\prime}\right\rangle}=s$, condition (i) guarantees that $\pi_{\downarrow}^{s^{\prime} c}\left(y_{\left\langle s^{\prime}\right\rangle}\right) \geq s$, and the lemma is proven in this case.

If $y \geq s^{\prime}$, then $\pi_{\downarrow}^{c}(y)=s^{\prime} \pi_{\downarrow}^{s^{\prime} c s^{\prime}}\left(s^{\prime} y\right)$. Furthermore, since $y$ is an upper bound for $s$ and $s^{\prime}$, the join $s^{\prime} \vee s$ exists, and $\left(s^{\prime} \vee s\right) \leq y$. Thus $s^{\prime}\left(s^{\prime} \vee s\right) \leq s^{\prime} y$ by Proposition 2.18, Since $\ell\left(s^{\prime} y\right)<\ell(y)$, condition (ii) guarantees that $\Sigma\left(W, s^{\prime} c s^{\prime}, s^{\prime}\left(s^{\prime} \vee s\right), s^{\prime} y\right)$ is true, or in other words, that $\pi_{\downarrow}^{s^{\prime} c s^{\prime}}\left(s^{\prime} y\right) \geq \pi_{\downarrow}^{s^{\prime} c s^{\prime}}\left(s^{\prime}\left(s^{\prime} \vee s\right)\right)$. Since $s^{\prime}$ is final in $s^{\prime} c s^{\prime}$, the restriction of $s^{\prime} c s^{\prime}$ to the parabolic subgroup generated by $s$ and $s^{\prime}$ is $s s^{\prime}$. Thus Proposition 6.10implies that $\pi_{\downarrow}^{s^{\prime} c s^{\prime}}\left(s^{\prime}\left(s^{\prime} \vee s\right)\right)=\pi_{\downarrow}^{s s^{\prime}}\left(s^{\prime}\left(s^{\prime} \vee s\right)\right)$. The unique reduced word for $s^{\prime}\left(s^{\prime} \vee s\right)$ is an initial segment of $s s^{\prime} s s^{\prime} s s^{\prime} \cdots$, and therefore $s^{\prime}\left(s^{\prime} \vee s\right)$ is $s s^{\prime}$-sortable. In particular, $\pi_{\downarrow}^{s s^{\prime}}\left(s^{\prime}\left(s^{\prime} \vee s\right)\right)=s^{\prime}\left(s^{\prime} \vee s\right)$.

Combining the inequalities of the previous paragraph, we have $\pi_{\downarrow}^{s^{\prime} c s^{\prime}}\left(s^{\prime} y\right) \geq$ $s^{\prime}\left(s^{\prime} \vee s\right)$. Proposition 6.9 implies that $\pi_{\downarrow}^{s^{\prime} c s^{\prime}}\left(s^{\prime} y\right) \geq s^{\prime}$, so $s^{\prime} \pi_{\downarrow}^{s^{\prime} c s^{\prime}}\left(s^{\prime} y\right) \geq s^{\prime} \vee s$ by Proposition 2.18. Therefore, $\pi_{\downarrow}^{c}(y)=s^{\prime} \pi_{\downarrow}^{s^{\prime} c s^{\prime}}\left(s^{\prime} y\right) \geq s^{\prime} \vee s \geq s$.

We now prove Theorem 6.1.

Proof of Theorem 6.1. Let $x, y \in W$ with $x \leq y$. We wish to show that $\pi_{\downarrow}^{c}(x) \leq$ $\pi_{\downarrow}^{c}(y)$. The proof is by induction on rank and on the length of $y$, the rank staying fixed whenever we appeal to induction on length. Note that we may immediately reduce to the case that $x \lessdot y$. Let $s$ be initial in $c$. Since $x \leq y$, it is impossible to have $x \geq s$ and $y \geq s$. Thus we must consider three cases.

Case 1: $y \nsupseteq s$ and $x \nsupseteq s$. Then $\pi_{\downarrow}^{c}(x)=\pi_{\downarrow}^{s c}\left(x_{\langle s\rangle}\right)$ and $\pi_{\downarrow}^{c}(y)=\pi_{\downarrow}^{s c}\left(y_{\langle s\rangle}\right)$. Because $x_{\langle s\rangle}$ is covered by or equal to $y_{\langle s\rangle}$, induction on rank shows that $\pi_{\downarrow}^{c}(x) \leq \pi_{\downarrow}^{c}(y)$.

Case 2: $x \geq s$ and $y \geq s$. Then $\pi_{\downarrow}^{c}(x)=s \cdot \pi_{\downarrow}^{s c s}(s x)$ and $\pi_{\downarrow}^{c}(y)=s \cdot \pi_{\downarrow}^{s c s}(s y)$. Since $s x \lessdot s y$, induction on length shows that $\pi_{\downarrow}^{s c s}(s x) \leq \pi_{\downarrow}^{s c s}(s y)$, and thus $\pi_{\downarrow}^{c}(x) \leq$ $\pi_{\downarrow}^{c}(y)$.

Case 3: $x \geq s$ and $y \geq s$. This case is illustrated in Figure 2. The dashed line separates $W_{\geq s}$ from $W_{\nsucceq s}$; the shaded region is $W_{\langle s\rangle}$; thick lines indicate cover relations and ordinary lines indicate inequalities. Many of the relations shown are not obvious and will be proved in the course of the proof.

Since $x \lessdot y$, Proposition 2.18 states that $x=s y$, and in particular $s$ is a cover reflection of $y$. Condition (i) of Lemma 6.12 holds by induction on rank, and condition (ii) holds by induction on length, with rank staying fixed. We conclude that $\Sigma(W, s c s, s, y)$ is true, or in other words that $\pi_{\downarrow}^{s c s}(y) \geq s$.

The fact that $x \nsupseteq s$ implies, by Proposition 6.7 that $\pi_{\downarrow}^{s c s}(x) \nsupseteq s$. Together with the relation $\pi_{\downarrow}^{s c s}(y) \geq s$ from the previous paragraph, this implies that $\pi_{\downarrow}^{s c s}(x) \neq$ $\pi_{\downarrow}^{s c s}(y)$. Since $\pi_{\downarrow}^{s c s}(x) \leq x<y$, we can apply Proposition 6.11 to conclude that 


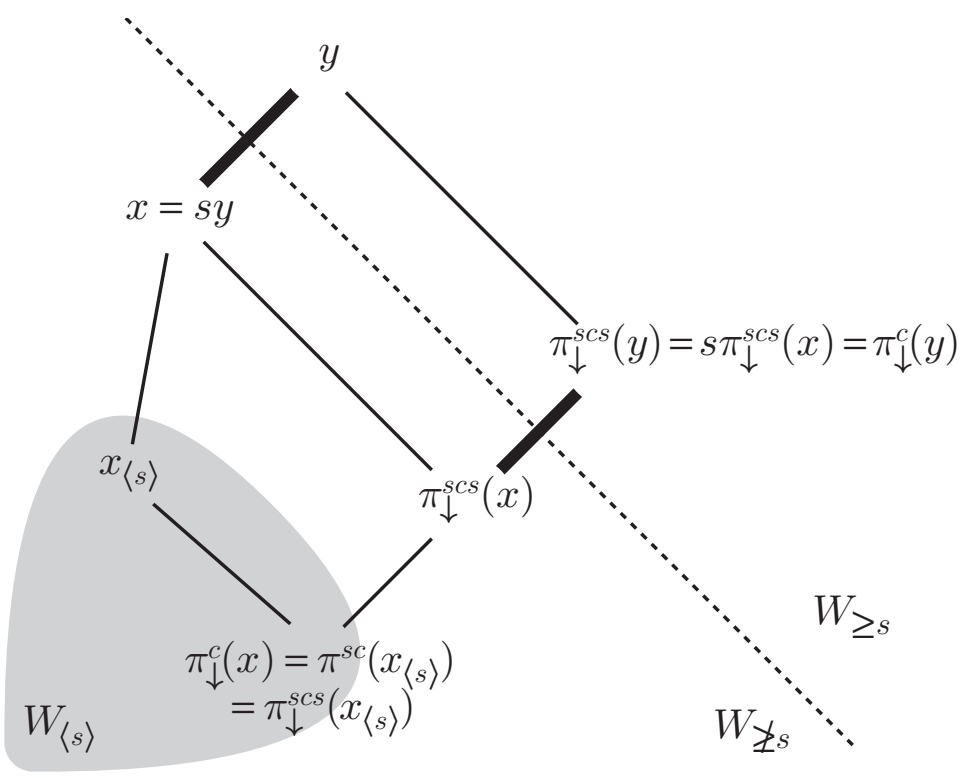

Figure 2. Case 3 of the proof of Theorem 6.1

$x D \subseteq \mathrm{Cone}_{s c s}\left(\pi_{\downarrow}^{s c s}(x)\right)$ but that $y D \nsubseteq \mathrm{Cone}_{s c s}\left(\pi_{\downarrow}^{s c s}(x)\right)$. Thus there is some $\beta \in$ $C_{s c s}\left(\pi_{\downarrow}^{s c s}(x)\right)$ such that interior points $p^{*}$ of $x D$ have $\left\langle p^{*}, \beta\right\rangle>0$ while interior points $p^{*}$ of $y D$ have $\left\langle p^{*}, \beta\right\rangle<0$. The only hyperplane separating $x D$ from $y D$ is the hyperplane orthogonal to $\alpha_{s}$. We therefore conclude that $\alpha_{s} \in C_{s c s}\left(\pi_{\downarrow}^{s c s}(x)\right)$. Now $-\alpha_{s}=s \alpha_{s} \in C_{c}\left(s \cdot \pi_{\downarrow}^{s c s}(x)\right)$ by the definition of $C_{c}$. Proposition 5.2 now shows that $s$ is a cover reflection of $s \cdot \pi_{\downarrow}^{s c s}(x)$, which equals $s \cdot \pi_{\downarrow}^{s c s}(s y)=\pi_{\downarrow}^{c}(y)$. We have therefore shown that $\pi_{\downarrow}^{c}(y)>s \pi_{\downarrow}^{c}(y)=\pi_{\downarrow}^{s c s}(s y)=\pi_{\downarrow}^{s c s}(x)$. By induction on length, $\pi_{\downarrow}^{s c s}(x) \geq \pi_{\downarrow}^{s c s}\left(x_{\langle s\rangle}\right)$ which, according to Proposition 6.10, equals $\pi_{\downarrow}^{s c}\left(x_{\langle s\rangle}\right)$. The latter is, by definition, $\pi_{\downarrow}^{c}(x)$.

Corollary 6.2 now follows immediately.

Proof of Corollary 6.2. By Propositions 6.7 and 6.8, the element $\pi_{\downarrow}^{c}(w)$ is $c$-sortable and is below $w$ in the weak order. If $v$ is any other $c$-sortable element below $w$ in the weak order, Theorem 6.1 implies that $\pi_{\downarrow}^{c}(v) \leq \pi_{\downarrow}^{c}(w)$, and since $v$ is $c$-sortable, $\pi_{\downarrow}^{c}(v)=v$ by the second half of Proposition 6.7

Next, we complete the proof of Theorem 6.3.

Proof of Theorem 6.3, In the proof of Proposition 6.11, the additional hypothesis $v \leq w$ was used only to rule out Case 4 and to provide the inequality (analogous to $v \leq w$ ) needed to appeal to induction. Thus, to prove Theorem 6.3. we need only establish Case 4 (assuming inductively that Theorem 6.3 holds for all examples of lesser length and rank) and copy the proofs of Cases 1,2 and 3 from the proof of Proposition 6.11. 
Suppose $v \geq s$ and $w \nsupseteq s$. Then $\pi_{\downarrow}^{c}(w) \neq v$ by Proposition 6.7. Since $s w \geq s$, Theorem 6.1 implies that $\pi_{\downarrow}^{s c s}(s w) \geq \pi_{\downarrow}^{s c s}(s)=s$. In particular, $\pi_{\downarrow}^{s c s}(s w) \neq s v$. Induction on the length of $v$ establishes that $s w D \nsubseteq \operatorname{Cone}_{s c s}(s v)=s \operatorname{Cone}_{c}(v)$. Thus $w D \nsubseteq$ Cone $_{c}(v)$ as desired.

We conclude this section with another corollary to Theorem 6.1 which considerably strengthens Proposition 6.10 .

Proposition 6.13. Let $J \subseteq S$ and let $c^{\prime}$ be the restriction of $c$ to $W_{J}$. Then $\pi_{\downarrow}^{c^{\prime}}\left(w_{J}\right)=\pi_{\downarrow}^{c}(w)_{J}$ for any $w \in W$.

Proof. Since $w \geq w_{J}$, Theorem 6.1 implies that $\pi_{\downarrow}^{c}(w) \geq \pi_{\downarrow}^{c}\left(w_{J}\right)$. By Proposition 6.10, the latter equals $\pi_{\downarrow}^{c^{\prime}}\left(w_{J}\right)$. Since the projection $x \mapsto x_{J}$ is order preserving, $\pi_{\downarrow}^{c}(w)_{J} \geq \pi_{\downarrow}^{c^{\prime}}\left(w_{J}\right)_{J}=\pi_{\downarrow}^{c^{\prime}}\left(w_{J}\right)$.

On the other hand, since $w \geq \pi_{\downarrow}^{c}(w)$, we have $w_{J} \geq\left(\pi_{\downarrow}^{c}(w)\right)_{J}$. Applying the order preserving map $\pi_{\downarrow}^{c^{\prime}}$ to both sides, $\pi_{\downarrow}^{c^{\prime}}\left(w_{J}\right) \geq \pi_{\downarrow}^{c^{\prime}}\left(\left(\pi_{\downarrow}^{c}(w)_{J}\right)\right.$. But $\left(\pi_{\downarrow}^{c}(w)\right)_{J}$ is $c^{\prime}$-sortable by Propositions 6.7 and 3.13 so $\pi_{\downarrow}^{c^{\prime}}\left(\left(\pi_{\downarrow}^{c}(w)\right)_{J}\right)=\left(\pi_{\downarrow}^{c}(w)\right)_{J}$. Thus $\pi_{\downarrow}^{c^{\prime}}\left(w_{J}\right) \geq \pi_{\downarrow}^{c}(w)_{J}$, so that $\pi_{\downarrow}^{c^{\prime}}\left(w_{J}\right)=\pi_{\downarrow}^{c}(w)_{J}$.

\section{Part II: The Cambrian Semilattice And FAN}

\section{The Cambrian semilattice}

In this section, we begin to establish the lattice-theoretic properties of $c$-sortable elements. We begin by listing and explaining the main results of the section.

Theorem 7.1. Let $A$ be a collection of c-sortable elements of $W$. If $A$ is nonempty, then $\bigwedge A$ is c-sortable. If $A$ has an upper bound, then $\bigvee A$ is c-sortable.

When $W$ is finite, $\bigvee S=w_{0}$, the longest element of $W$. Since each $s \in S$ is $c$ sortable, we have the following corollary to Theorem [7.1] (Cf. [32, Corollary 4.4].)

Corollary 7.2. If $W$ is finite, then $w_{0}$ is c-sortable for any Coxeter element $c$.

The statement about meets in Theorem 7.1 means that the restriction of the weak order to $c$-sortable elements is a sub-(meet-)semilattice of the weak order on all of $W$. This sub-semilattice will be called the c-Cambrian semilattice. By Theorem 6.1, for any $w \in W$, the restriction of the weak order to $c$-sortable elements weakly below $w$ is the interval $\left[e, \pi_{\downarrow}^{c}(w)\right]$ in the $c$-Cambrian semilattice. Theorem 7.1 implies that the interval $\left[e, \pi_{\downarrow}^{c}(w)\right]$ in the $c$-Cambrian semilattice is a sublattice of the interval $[e, w]$ in the weak order. In particular, when $W$ is finite, the $c$-Cambrian semilattice is called the $c$-Cambrian lattice and is a sublattice of the weak order on $W$.

Remarkably, the $c$-Cambrian semilattice is not only a subobject of the weak order, but also a quotient object.

Theorem 7.3. For $A$ any subset of $W$, if $A$ is nonempty, then $\bigwedge \pi_{\downarrow}^{c}(A)=\pi_{\downarrow}^{c}(\bigwedge A)$, and if $A$ has an upper bound, then $\bigvee \pi_{\downarrow}^{c}(A)=\pi_{\downarrow}^{c}(\bigvee A)$. 
Theorem 7.3 means that $\pi_{\downarrow}^{c}$ is a semilattice homomorphism from the weak order on $W$ to the $c$-Cambrian semilattice and that the restriction of $\pi_{\downarrow}^{c}$ to any interval $[1, w]$ is a lattice homomorphism from $[1, w]$ to the interval $\left[1, \pi_{\downarrow}^{c}(w)\right]$ in the $c$ Cambrian semilattice. In particular, when $W$ is finite, $\pi_{\downarrow}^{c}$ is a lattice homomorphism from the weak order on $W$ to the $c$-Cambrian lattice.

As a consequence, the $c$-Cambrian semilattice can also be realized as the meetsemilattice quotient of the weak order on $W$ modulo the lattice congruence defined by the fibers of $\pi_{\downarrow}^{c}$. As such, the $c$-Cambrian semilattice is the partial order on the fibers of $\pi_{\downarrow}^{c}$ defined as follows: A fiber $F_{1}$ is below a fiber $F_{2}$ if and only if there exists elements $x_{1} \in F_{1}$ and $x_{2} \in F_{2}$ with $x_{1} \leq x_{2}$ in the weak order. In fact, since each fiber has a unique minimal element, the partial order on fibers can also be defined by a formally weaker requirement: A fiber $F_{1}$ is below a fiber $F_{2}$ if and only if, for every $x_{2} \in F_{2}$, there exists $x_{1} \in F_{1}$ with $x_{1} \leq x_{2}$ in the weak order. Not surprisingly, the dual version of this latter characterization does not hold: It is possible to find fibers $F_{1} \leq F_{2}$ and an element $x_{1} \in F_{1}$ which is below no element of $F_{2}$. For example, take $W$ with $S=\left\{s_{1}, s_{2}, s_{3}\right\}$ and $m\left(s_{1}, s_{2}\right)=m\left(s_{1}, s_{3}\right)=m\left(s_{2}, s_{3}\right)=\infty$ and set $c=s_{1} s_{2} s_{3}$. Then the fiber $F_{1}=\left(\pi_{\downarrow}^{c}\right)^{-1}\left(s_{2}\right)$ contains the element $s_{2} s_{1}$. The fiber $F_{2}=\left(\pi_{\downarrow}^{c}\right)^{-1}\left(s_{2} s_{3}\right)$ has $F_{2} \geq F_{1}$, but no element of $F_{2}$ is above $s_{2} s_{1}$.

There is also a geometric characterization of the $c$-Cambrian semilattice, inherited from the geometric characterization of the weak order which we now review. Let $b^{*}$ be a vector in the interior of $D$. All cover relations of the weak order on $W$ are obtained as follows: Suppose $w_{1} D$ and $w_{2} D$ are adjacent and let $n \in V$ be a normal vector to their common boundary hyperplane, with $n$ pointing in the direction of $w_{2} D$. The characterization of the weak order in terms of inversion sets implies that $w_{1} D \lessdot w_{2} D$ if and only if $\left\langle b^{*}, n\right\rangle<0$. In light of Lemma 5.6] and Theorem 6.3. the $c$-Cambrian semilattice is the partial order on the cones Cone $_{c}(v)$ with covers defined analogously in terms of the same vector $b^{*}$.

Another important lattice-theoretic property of $c$-sortable elements is the following theorem, which was pointed out for finite $W$ in [33, Remark 3.8]. Although the proof of Theorem 7.4 depends (via Theorem 7.1) on most of the machinery developed thus far, if Theorem 7.4 were known from the beginning, almost all of the preceding results would follow easily.

Theorem 7.4. If $s$ is initial in $c$, then the map

$$
v \mapsto\left\{\begin{array}{cl}
s v & \text { if } v \geq s, \\
s \vee v & \text { if } v \nsupseteq s
\end{array}\right.
$$

is a bijection from the set $\{c$-sortable elements $v$ such that $s \vee v$ exists $\}$ to the set of all scs-sortable elements. The inverse map is

$$
x \mapsto\left\{\begin{array}{cl}
s x & \text { if } x \geq s, \\
x_{\langle s\rangle} & \text { if } x \geq s .
\end{array}\right.
$$

Theorem 7.4 is illustrated pictorially in Section 10.1. especially Figures 3 and 4 . We now proceed to prove Theorems 7.1, 7.3 and 7.4.

Proof of Theorem 7.1 . First consider the assertion about $\wedge A$. Define $I$ to be $\bigcap_{a \in A} \operatorname{inv}(a)$. Let $W^{\prime}$ be a noncommutative generalized rank two parabolic subgroup of $W$ with canonical generators $r_{1}$ and $r_{2}$ and with $\omega_{c}\left(\beta_{r_{1}}, \beta_{r_{2}}\right)>0$. Then $I \cap W^{\prime}=$ $\bigcap_{a \in A}\left(\operatorname{inv}(a) \cap W^{\prime}\right)$, and every set in the latter intersection is either the empty set, the singleton $\left\{r_{2}\right\}$ or a set of the form $\left\{r_{1}, r_{1} r_{2} r_{1}, r_{1} r_{2} r_{1} r_{2} r_{1}, \ldots, r_{1} r_{2} \cdots r_{2} r_{1}\right\}$. 
Thus, the intersection $I \cap W^{\prime}$ is also of one of these forms. If $\omega_{c}\left(\beta_{r_{1}}, \beta_{r_{2}}\right)=0$, then a slight modification of this argument shows that $I \cap W^{\prime}$ is either $\emptyset,\left\{r_{1}\right\}$ or $\left\{r_{2}\right\}$. Thus, from Lemma 2.17 we see that $I$ is the set of inversions of some element $w$ of $W$. Necessarily, $w=\bigwedge A$. Moreover, we see that $w$ is $c$-aligned and hence, by Theorem 4.3, $w$ is $c$-sortable.

We now prove the assertion about $\bigvee A$. Let $w=\bigvee A$, which exists since we assumed that $A$ has an upper bound. For every $a \in A$, we have $\pi_{\downarrow}^{c}(w) \geq \pi_{\downarrow}^{c}(a)=a$, where the inequality is by Theorem 6.1 and the equality is by Proposition 6.7 So $\pi_{\downarrow}^{c}(w) \geq \bigvee A=w$. Then, by Proposition 6.7, $\pi_{\downarrow}^{c}(w)=w$, so $w=\bigvee A$ is $c$-sortable.

Remark 7.5. The proof of Theorem 7.1 shows that, for any nonempty set $A$ of $c$-sortable elements, $\operatorname{inv}\left(\bigwedge_{a \in A} a\right)=\bigcap_{a \in A} \operatorname{inv}(a)$. This is not true for arbitrary nonempty subsets $A$ of $W$.

Proof of Theorem 17.3. Suppose $A$ is nonempty so that $\bigwedge A$ exists. For each $a \in A$, we have $a \geq \bigwedge A$ so that $\pi_{\downarrow}^{c}(a) \geq \pi_{\downarrow}^{c}(\bigwedge A)$ by Theorem 6.1. Thus $\bigwedge \pi_{\downarrow}^{c}(A) \geq$ $\pi_{\downarrow}^{c}(\bigwedge A)$.

However, $\bigwedge \pi_{\downarrow}^{c}(A) \leq \bigwedge A$ because $\pi_{\downarrow}^{c}(a) \leq a$ for each $a$ in $A$. Thus by Theorem 6.1 $\pi_{\downarrow}^{c}\left(\bigwedge \pi_{\downarrow}^{c}(A)\right) \leq \pi_{\downarrow}^{c}(\bigwedge A)$. But Theorem 7.1 states that $\bigwedge \pi_{\downarrow}^{c}(A)$ is $c$-sortable, so that $\pi_{\downarrow}^{c}\left(\bigwedge \pi_{\downarrow}^{c}(A)\right)=\bigwedge \pi_{\downarrow}^{c}(A)$ and $\bigwedge \pi_{\downarrow}^{c}(A) \leq \pi_{\downarrow}^{c}(\bigwedge A)$. Thus $\bigwedge \pi_{\downarrow}^{c}(A)=\pi_{\downarrow}^{c}(\bigwedge A)$.

Now, consider the assertion about joins. If $A$ has an upper bound $b$, then since the set of elements below $b$ is finite, $A$ is finite. Thus it is enough to prove, for any $x$ and $y$ in $W$ such that $x \vee y$ exists, that $\pi_{\downarrow}^{c}(x) \vee \pi_{\downarrow}^{c}(y)=\pi_{\downarrow}^{c}(x \vee y)$. Let $s$ be initial in $c$. We first prove a special case of the result:

Special Case: $x=s$ and $y \geq s$. In this case, $s$ is a cover reflection of $s \vee y$ by Lemma 2.23. By Theorem 6.1, $\pi_{\downarrow}^{c}(s \vee y)$ is above $s$, and by Proposition 6.7. $\pi_{\downarrow}^{c}(s(s \vee y))$ is not above $s$. Thus by Theorem 6.3, the hyperplane $H_{s}$ defines a wall of $\mathrm{Cone}_{c}\left(\pi_{\downarrow}^{c}(s \vee y)\right)$, so $-\alpha_{s} \in A_{c}\left(\pi_{\downarrow}^{c}(s \vee y)\right)$. Proposition 5.2 implies that $s$ is a cover reflection of $\pi_{\downarrow}^{c}(s \vee y)$. Now Proposition 5.4 implies that every other cover reflection of $\pi_{\downarrow}^{c}(s \vee y)$ is in $W_{\langle s\rangle}$, so that $\pi_{\downarrow}^{c}(s \vee y)=s \vee\left(\left[\pi_{\downarrow}^{c}(s \vee y)\right]_{\langle s\rangle}\right)$ by Lemma 2.22. By Propositions 6.13 and 2.20 we have

$$
\left[\pi_{\downarrow}^{c}(s \vee y)\right]_{\langle s\rangle}=\pi_{\downarrow}^{s c}\left((s \vee y)_{\langle s\rangle}\right)=\pi_{\downarrow}^{s c}\left(s_{\langle s\rangle} \vee y_{\langle s\rangle}\right)=\pi_{\downarrow}^{s c}\left(1 \vee y_{\langle s\rangle}\right)=\pi_{\downarrow}^{s c}\left(y_{\langle s\rangle}\right),
$$

which is $\pi_{\downarrow}^{c}(y)$ by Proposition 2.29, so $\pi_{\downarrow}^{c}(s \vee y)=s \vee\left(\left[\pi_{\downarrow}^{c}(s \vee y)\right]_{\langle s\rangle}\right)=\pi_{\downarrow}^{c}(s) \vee$ $\pi_{\downarrow}^{c}(y)$.

We now prove the general result by induction on rank and on the length of $x \vee y$. We break into several cases.

Case 1: $x \geq s$ and $y \geq s$. In this case, $x \vee y \geq s$. Thus $\pi_{\downarrow}^{c}(x)=s \pi_{\downarrow}^{s c s}(s x)$, $\pi_{\downarrow}^{c}(y)=s \pi_{\downarrow}^{s c s}(s y)$ and $\pi_{\downarrow}^{c}(x \vee y)=s \pi_{\downarrow}^{s c s}(s(x \vee y))$. Now $s x \vee s y=s(x \vee y)$ has length $\ell(x \vee y)-1$, so by induction on $\ell(x \vee y)$ we obtain $\pi_{\downarrow}^{s c s}(s x) \vee \pi_{\downarrow}^{s c s}(s y)=\pi_{\downarrow}^{s c s}(s x \vee s y)$. 
Thus $\pi_{\downarrow}^{c}(x) \vee \pi_{\downarrow}^{c}(y)$ is

$$
\left(s \pi_{\downarrow}^{s c s}(s x)\right) \vee\left(s \pi_{\downarrow}^{s c s}(s y)\right)=s\left(\pi_{\downarrow}^{s c s}(s x) \vee \pi_{\downarrow}^{s c s}(s y)\right)=s \pi_{\downarrow}^{s c s}(s x \vee s y),
$$

which equals $s \pi_{\downarrow}^{s c s}(s(x \vee y))=\pi_{\downarrow}^{c}(x \vee y)$.

Case 2: $x \nsupseteq s$ and $y \nsupseteq s$. Then $(x \vee y) \nsupseteq s$ by Corollary 2.21 We have $\pi_{\downarrow}^{c}(x)=$ $\pi_{\downarrow}^{s c}\left(x_{\langle s\rangle}\right), \pi_{\downarrow}^{c}(y)=\pi_{\downarrow}^{s c}\left(y_{\langle s\rangle}\right)$ and $\pi_{\downarrow}^{c}(x \vee y)=\pi_{\downarrow}^{s c}\left((x \vee y)_{\langle s\rangle}\right)$. The latter equals $\pi_{\downarrow}^{s c}\left(x_{\langle s\rangle} \vee y_{\langle s\rangle}\right)$ by Proposition 2.20, By induction on rank, $\pi_{\downarrow}^{s c}\left(x_{\langle s\rangle}\right) \vee \pi_{\downarrow}^{s c}\left(y_{\langle s\rangle}\right)=$ $\pi_{\downarrow}^{s c}\left(x_{\langle s\rangle} \vee y_{\langle s\rangle}\right)$, so $\pi_{\downarrow}^{c}(x) \vee \pi_{\downarrow}^{c}(y)=\pi_{\downarrow}^{c}(x \vee y)$.

Case 3: Exactly one of $x$ and $y$ is above $s$. Without loss of generality, we take $x \geq s$ and $y \nsupseteq s$. Let $z=s \vee y$, so that $x \vee y=x \vee z$ and $\pi_{\downarrow}^{c}(x \vee y)=\pi_{\downarrow}^{c}(x \vee z)$. Since $s x \vee s z=s(x \vee z)$ has length $\ell(x \vee z)-1=\ell(x \vee y)-1$, we argue by induction on $\ell(x \vee y)$ as in Case 1 to conclude that $\pi_{\downarrow}^{c}(x \vee z)=\pi_{\downarrow}^{c}(x) \vee \pi_{\downarrow}^{c}(z)$. Now $\pi_{\downarrow}^{c}(z)=$ $\pi_{\downarrow}^{c}(s \vee y)=\pi_{\downarrow}^{c}(s) \vee \pi_{\downarrow}^{c}(y)$ by the Special Case. Thus $\pi_{\downarrow}^{c}(x \vee y)=\pi_{\downarrow}^{c}(x) \vee \pi_{\downarrow}^{c}(s) \vee \pi_{\downarrow}^{c}(y)$. Since $\pi_{\downarrow}^{c}(s) \leq \pi_{\downarrow}^{c}(x)$ by Theorem 6.1, we obtain $\pi_{\downarrow}^{c}(x \vee y)=\pi_{\downarrow}^{c}(x) \vee \pi_{\downarrow}^{c}(y)$.

Proof of Theorem 7.4. Proposition 2.29 implies that the involution $v \mapsto s v$ is a bijection between $c$-sortable elements above $s$ and scs-sortable elements not above $s$. Thus it remains to show that $v \mapsto s \vee v$ is a bijection from $c$-sortable elements $v$ with $v \nsupseteq s$ such that $s \vee v$ exists to scs-sortable elements $x$ above $s$ and that the inverse map is $x \mapsto x_{\langle s\rangle}$.

If $v$ is $c$-sortable with $v \nsupseteq s$, then by Proposition 2.29 $v$ is an $s c$-sortable element of $W_{\langle s\rangle}$. In particular, $v$ is an scs-sortable element of $W$ by Proposition 2.30. Thus Theorem 7.1 now implies that $s \vee v$ is an $s c s$-sortable element (necessarily above $s$ ). By Proposition 2.20, $(s \vee v)_{\langle s\rangle}=s_{\langle s\rangle} \vee v_{\langle s\rangle}=1 \vee v=v$.

If $x$ is $s c s$-sortable with $x \geq s$, then since $s$ is final in $s c s, x_{\langle s\rangle}$ is $s c$-sortable by Proposition 3.13. In particular, $x_{\langle s\rangle}$ is a $c$-sortable element not above $s$. Furthermore, Proposition 5.3 states that $x=s \vee x_{\langle s\rangle}$.

\section{CANONICAL Join REPRESENTATIONS}

In this section we consider a lattice theoretic construction which has important consequences for sortable elements, particularly as they relate to noncrossing partitions. Specifically, we consider a canonical way of representing an element $w$ of the weak order as the join of an antichain $A$ of join-irreducible elements. If $A$ is the unique minimal (in a sense made precise below) antichain whose join is $w$, then $A$ is called the canonical join representation of $w$. The notion of canonical join representations is a key component of the theory of free lattices (see [19]). We will prove the following theorem.

Theorem 8.1. Every $w \in W$ has a canonical join representation A. Furthermore, $\operatorname{cov}(w)$ is the disjoint union, over all $j \in A$, of $\operatorname{cov}(j)$.

Note that since each $j \in A$ is join-irreducible, each $\operatorname{set} \operatorname{cov}(j)$ is a singleton. This theorem implies, by a well-known fact [19, Theorem 2.24] from the theory of free lattices and by Proposition 2.19, that every interval in the weak order is a semidistributive lattice in the sense of [19, Section I.3]. A stronger result was proven, for $W$ finite, in [11]. Cf. [10, 28].

More importantly for our purposes, canonical join representations of sortable elements are well-behaved in the following sense. 
Proposition 8.2. If $v \in W$ is c-sortable, then every element of its canonical join representation is c-sortable.

Call a reflection c-accessible if it occurs in the inversion set of some $c$-sortable element of $W$. Proposition 8.2 leads to the following theorem, which establishes a bijection between $c$-accessible reflections and $c$-sortable join-irreducible elements.

Theorem 8.3. Let $t$ be any c-accessible reflection in $W$. Then there exists a unique $c$-sortable join-irreducible $j$ whose unique cover reflection is $t$. Furthermore, $j$ is the unique minimal element among c-sortable elements having $t$ as an inversion.

For examples illustrating Theorems 8.1 and 8.3 and Proposition 8.2 . see Section 10.1. Theorems 8.1 and 8.3 and Proposition 8.2 also give greater insight into a map nc ${ }_{c}$ defined on $c$-sortable elements. When $W$ is finite, $\mathrm{nc}_{c}$ is a bijection from $c$ sortable elements to $c$-noncrossing partitions [32, Theorem 6.1]. At the end of this section, we recall the definition of noncrossing partitions and of $\mathrm{nc}_{c}$ and explain how Theorem 8.1 and Proposition 8.2 lead to the insight that, for finite $W$, noncrossing partitions encode the canonical join representations of sortable elements. For infinite $W$, there is not yet a consensus on the right definition of noncrossing partitions, so we cannot generalize the result that $\mathrm{nc}_{c}$ is a bijection. However, we show in Theorem 8.9 that $\mathrm{nc}_{c}$, and several variants of $\mathrm{nc}_{c}$, are injective.

We now proceed to give details and proofs. Recall from Section 2 that a partially ordered set $P$ is finitary if, for every $x \in P$, the set of elements less than or equal to $x$ is finite. If $L$ is a finitary meet-semilattice, then $L$ has a unique minimal element, denoted $\hat{0}$, and furthermore, by a standard argument, for every $x$, the interval $[\hat{0}, x]$

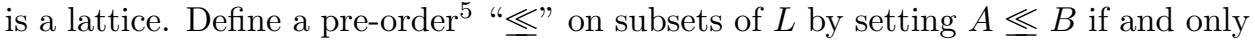
if for every $a \in A$ there exists $b \in B$ with $a \leq b$. Notice that if $A \leq B$ and $\bigvee B$ exists, then $\bigvee A$ exists and $\bigvee A \leq \bigvee B$ in $L$.

For $A \subseteq L$, we write $I(A)$ for the (lower) order ideal generated by $A$. Another description of the relation $\leq$ is that $A \leq B$ if and only if $I(A) \subseteq I(B)$. Any finite order ideal is of the form $I(A)$ for precisely one antichain $A$. Thus $\leq$ induces a partial order (rather than just a preorder) on the finite antichains of $L$.

An antichain $A \subseteq L$ is called the canonical join representation of $x$ if:

(i) $x=\bigvee A$, and

(ii) if $B$ is any other antichain in $L$ with $\bigvee B=x$, then $A \leqslant B$.

The canonical join representation of $x$, if it exists, is the unique minimal antichain, with respect to $\ll$, among antichains joining to $x$. Since $L$ is finitary, the canonical join representation of $x$ is a finite set.

Recall also that an element $j \in L$ is called join-irreducible if any finite set $X$ with $j=\bigvee X$ has $j \in X$ or equivalently if $j$ covers exactly one element of $L$.

Lemma 8.4. If $A$ is the canonical join representation of some $x \in L$, then every element of $A$ is join-irreducible.

Proof. Suppose $A$ is an antichain with $\bigvee A=x$. If some $j \in A$ is not joinirreducible, then $j=\bigvee X$ for some $X \subseteq L$ with $j \notin X$. Since $L$ is finitary, $X$ is finite. The set $A^{\prime}$ of maximal elements of $X \cup(A \backslash\{j\})$ is a finite antichain joining to $x$. But no element of $A^{\prime}$ is above $j$, so $A \not A^{\prime}$, and thus $A$ is not the canonical join representation of $x$.

\footnotetext{
${ }^{5} \mathrm{~A}$ preorder is a relation that is reflexive and transitive but may not be antisymmetric.
} 
Remark 8.5. Our development of canonical join representations is loosely modeled on the treatment in [19, Section II.1]. One difference is that we use the symbol " $\leq$ " in place of "《" to emphasize the fact that $A \lll A$ for any $A \subseteq L$.

The weak order is finitary, so the general notions outlined above apply. The proof of Theorem 8.1 begins with the following observation.

Lemma 8.6. Let $w \in W$ and let $t$ be a cover reflection of $w$. Then there is some $j(w, t)$ in $W$ such that the set $\{v \in W: v \leq w$ and $v \not \leq t w\}$ is equal to the interval $[j(w, t), w]$.

Proof. Applying Proposition 2.19, the lemma is equivalent to the statement that the (lower) order ideal $\left\{v \in W: v \leq w^{-1}\right.$ and $\left.v \nsupseteq w^{-1} t w\right\}$ has a unique maximal element. But $t w \lessdot w$, so $t w=w s$, and $w^{-1} t w=s$ for some simple generator $s$. Now Corollary 2.21 implies that $\left\{v \in W: v \leq w^{-1}\right.$ and $\left.v \nsupseteq w^{-1} t w\right\}$ is closed under joins. Since this set is finite, it has a unique maximal element.

To prove Theorem 8.1 we show that the set $\{j(w, t): t \in \operatorname{cov}(w)\}$ is the canonical join representation of $w$.

Proof of Theorem 8.1. Let $A$ stand for the set $\{j(w, t): t \in \operatorname{cov}(w)\}$. First, we check that $A$ is an antichain. Suppose $t, t^{\prime} \in \operatorname{cov}(w)$ with $t \neq t^{\prime}$. Then $\operatorname{inv}(t w)=$ $\operatorname{inv}(w) \backslash\{t\}$ and $\operatorname{inv}\left(t^{\prime} w\right)=\operatorname{inv}(w) \backslash\left\{t^{\prime}\right\}$. In particular, $t^{\prime} w$ is an element of the set $\{v \in W: v \leq w$ and $v \not \leq t w\}$, so $t^{\prime} w \geq j(w, t)$. This implies that $j(w, t)$ is not in $\left\{v \in W: v \leq w\right.$ and $\left.v \not \leq t^{\prime} w\right\}=\left[j\left(w, t^{\prime}\right), w\right]$, and since $j(w, t) \leq w$, we have $j(w, t) \nsupseteq j\left(w, t^{\prime}\right)$.

Next, we check that $w=\bigvee A$. Note first that $w$ is an upper bound for $A$, so $\bigvee A$ exists and is weakly below $w$. Let $v$ be any element covered by $w$, so that in particular $v=t w$ for some $t \in \operatorname{cov}(w)$. Since $t$ is an inversion of $j(w, t)$ but $\operatorname{inv}(v)=\operatorname{inv}(w) \backslash\{t\}$, the element $v$ is not above $j(w, t)$. Thus $w$ is the minimal upper bound for $A$.

Now suppose that $w=\bigvee B$ for some antichain $B$ in $L$. We need to show that $A \ll B$. That is, we must show that for every $t \in \operatorname{cov}(w)$, there exists $b \in B$ with $j(w, t) \leq b$. Fix one such $t$. Since $t w<w$ and $\bigvee B=w$, we know that $t w$ is not an upper bound for $B$. Let $b \in B$ be such that $b \not \leq t w$. Then, since we know $b \leq w$, we have $j(w, t) \leq b$. This proves that $A \leq B$, thus completing the proof that $A$ is the canonical join representation of $w$.

In particular, Lemma 8.4 implies that each $j(w, t)$ is join-irreducible. Let $t^{\prime}$ be the unique cover reflection of $j(w, t)$. Since $j(w, t)$ is the unique minimal element of $\{v \in W: v \leq w$ and $v \not \leq t w\}$, we must have $t^{\prime} j(w, t) \leq t w$. But $\operatorname{inv}(t w)=\operatorname{inv}(w) \backslash$ $\{t\}$ and $\operatorname{inv}\left(t^{\prime} j(w, t)\right)=\operatorname{inv}(j(w, t)) \backslash\left\{t^{\prime}\right\}$, so $t=t^{\prime}$. This establishes the second assertion of the theorem.

Proposition 8.2 now follows easily from Theorem 7.3

Proof of Proposition 8.2. Let $v$ be $c$-sortable and let $A$ be its canonical join representation. Consider the set $\pi_{\downarrow}^{c}(A)=\left\{\pi_{\downarrow}^{c}(j): j \in A\right\}$. Theorem 7.3 implies that

$$
\bigvee\left(\pi_{\downarrow}^{c}(A)\right)=\pi_{\downarrow}^{c}(\bigvee A)=\pi_{\downarrow}^{c}(v)=v
$$

Let $B$ be the antichain of maximal elements of $\pi_{\downarrow}^{c}(A)$. In particular, $\bigvee B=v$. Since $A$ is the canonical join representation of $v$, we have $A \lll B$. But since each 
element of $B$ is $\pi_{\downarrow}^{c}(j)$ for some $j \in A$, we have $B \leq A$. Thus $A=B$, and therefore $A=\pi_{\downarrow}^{c}(A)$. Thus $j=\pi_{\downarrow}^{c}(j)$ for each $j \in A$.

If $v$ is a $c$-sortable join-irreducible element, then its unique cover reflection is, by definition, a $c$-accessible reflection. Theorem 8.3 states in particular that this map from $c$-sortable join-irreducible elements to $c$-accessible reflections is a bijection. We prove the theorem in two halves. The first half is proved by a simple inductive argument using no deep results.

Proposition 8.7. For any reflection $t$, there is at most one join-irreducible csortable element whose unique cover reflection is $t$.

Proof. Suppose $j_{1}$ and $j_{2}$ are join-irreducible $c$-sortable elements, each having $t$ as a cover reflection. We argue by induction on $\ell\left(j_{1}\right)$ and on the rank of $W$ that $j_{1}=j_{2}$. Let $s$ be initial in $c$.

If either join-irreducible (say $j_{1}$ ) is not above $s$, then it is in $W_{\langle s\rangle}$, so that in particular $t \in W_{\langle s\rangle}$. Therefore $j_{2}$ has the property that all of its cover reflections are in $W_{\langle s\rangle}$, and thus $j_{2}$ is also in $W_{\langle s\rangle}$ by Lemma 2.24. By induction on rank, $j_{1}=j_{2}$.

Otherwise both $j_{1}$ and $j_{2}$ are above $s$. If either (say $j_{1}$ ) equals $s$, then $t=s$, and Lemma 2.24 implies that $j_{2} \in W_{\{s\}}$, so that $j_{2}=s$ as well. If neither $j_{1}$ nor $j_{2}$ is $s$, then $s j_{1}$ and $s j_{2}$ are join-irreducible $s c s$-sortable elements having the same cover reflection. By induction on length, $s j_{1}=s j_{2}$, so $j_{1}=j_{2}$.

The second half of the proof of Theorem 8.3 is implied by the following proposition, which uses, via Proposition 8.2, the machinery developed over the course of the paper.

Proposition 8.8. Let $t$ be a c-accessible reflection and let $v$ be minimal in the weak order among c-sortable elements having $t \in \operatorname{inv}(v)$. Then $v$ is join-irreducible and has $t$ as its unique cover reflection.

Proof. If $t_{1}, t_{2}, \ldots, t_{k}$ is the sequence of reflections arising from a $c$-sorting word $s_{1} s_{2} \cdots s_{k}$ for $v$, then in particular $t=t_{k}$. (If not, then $t=t_{i}$ for some $i<k$, so that $s_{1} s_{2} \cdots s_{i}$ is a $c$-sortable element having $t$ as an inversion, contradicting the minimality of $v$.) Thus in particular, $t$ is a cover reflection of $v$. If $v$ has an additional cover reflection, then Proposition 8.2 and Theorem 8.1 imply that $v$ is the join of a set of two or more join-irreducible $c$-sortable elements strictly less than $v$, one of which has $t$ as a cover reflection. This contradicts minimality. Thus $v$ is a $c$-sortable join-irreducible element whose unique cover reflection is $t_{k}=t$.

We now describe noncrossing partitions and explain how they relate to the results of this section. Let $W$ be a finite Coxeter group. An element $w \in W$ can be written (typically in many ways) as a word in the alphabet $T$. We refer to words in the alphabet $T$ as $T$-words, to avoid confusion with words in the alphabet $S$, which are called simply "words" throughout the paper. A reduced T-word for $w$ is a $T$-word for $w$ which has minimal length among all $T$-words for $w$. Say $x \leq_{T} y$ if $x$ possesses a reduced $T$-word which is a prefix of some reduced $T$-word for $y$. The noncrossing partition lattice in $W$ (with respect to the Coxeter element $c$ ) is the interval $[1, c]_{T}$ in the partial order " $\leq_{T}$." The elements of this interval are called $c$-noncrossing partitions. 
Results of [9] show that a noncrossing partition is uniquely determined by its fixed space and that the fixed space $\operatorname{Fix}(x) \subseteq V^{*}$ of a noncrossing partition $x$ is the intersection $\bigcap_{i=1}^{k} H_{t_{i}}$, where $t_{1} \cdots t_{k}$ is any reduced $T$-word for $x$ and $H_{t}$ denotes the hyperplane fixed by $t$. The subspace $\bigcap_{i=1}^{k} H_{t_{i}}$ is uniquely defined by the associated noncrossing parabolic subgroup - the subgroup $\operatorname{Stab}\left(\bigcap_{i=1}^{k} H_{t_{i}}\right)$ fixing $\bigcap_{i=1}^{k} H_{t_{i}}$ pointwise.

In [32, a map $\mathrm{nc}_{c}$ was defined on $c$-sortable elements and was shown 32, Theorem 6.1] to be a bijection from $c$-sortable elements to $c$-noncrossing partitions. The definition of $\mathrm{nc}_{c}(v)$ is as follows: Let $t_{1}, t_{2}, \ldots, t_{k}$ be the reflection sequence for a $c$-sorting word for $v$. Since every cover reflection of $v$ is an inversion of $v$, the cover reflections of $v$ occur in the sequence $t_{1}, t_{2}, \ldots, t_{k}$. Let $u_{1}, u_{2}, \ldots, u_{r}$ be the cover reflections of $v$, ordered as a subsequence of $t_{1}, t_{2}, \ldots, t_{k}$. Then $\operatorname{nc}_{c}(v)=u_{1} u_{2} \cdots u_{r}$. The product $u_{1} u_{2} \cdots u_{r}$ is a reduced $T$-word for $\mathrm{nc}_{c}(v)$. By Lemma 2.27, the reflections $u_{1}, u_{2}, \ldots, u_{r}$ are the canonical generators of the parabolic subgroup associated to $\operatorname{nc}_{c}(v)$.

Theorem 8.3 allows us to identify (when $W$ is finite) the set $T$ of reflections of $W$ with the set of join-irreducible $c$-sortable elements of $W$. Under this identification, Theorem 8.1 and Proposition 8.2 imply that, if one specifies noncrossing partitions by the canonical generators of the associated parabolic subgroup, the noncrossing partitions are exactly the canonical join representations of sortable elements. Put another way, a set of reflections canonically generates a noncrossing parabolic subgroup if and only if the corresponding set of join-irreducible $c$-sortable elements canonically represents its join.

For general $W$, the map $\mathrm{nc}_{c}$ still makes sense, and our results easily imply that the map $\mathrm{nc}_{c}$ is injective. In fact, all of the natural variants of $\mathrm{nc}_{c}$ are injective, as made precise in the following theorem.

Theorem 8.9. The following maps are injective:

(i) The map $\mathrm{nc}_{c}$ from c-sortable elements of $W$ to $W$.

(ii) The map $v \mapsto \operatorname{Fix}\left(\operatorname{nc}_{c}(v)\right)$ from c-sortable elements of $W$ to subspaces of $V^{*}$.

(iii) The map $v \mapsto \operatorname{Stab}\left(\operatorname{Fix}\left(\operatorname{nc}_{c}(v)\right)\right)$ from c-sortable elements of $W$ to parabolic subgroups of $W$.

(iv) The map cov from c-sortable elements of $W$ to sets of reflections in $W$.

Proof. Suppose $v$ and $v^{\prime}$ are $c$-sortable elements with $\operatorname{cov}(v)=\operatorname{cov}\left(v^{\prime}\right)$. By Theorem 8.1 and Proposition [8.2, the canonical join representation of $v$ consists of $c$-sortable join-irreducible elements $j$, such that the set of cover reflections occurring as cover reflections of the $j$ 's is $\operatorname{cov}(v)$. The same is true of the canonical join representation of $v^{\prime}$, so by Theorem 8.3 , these two join representations coincide, and thus $v=v^{\prime}$. This is (iv).

Obviously, $\operatorname{nc}_{c}(v)$ determines $\operatorname{Fix}\left(\mathrm{nc}_{c}(v)\right)$, which determines $\operatorname{Stab}\left(\operatorname{Fix}\left(\mathrm{nc}_{c}(v)\right)\right)$. We conclude the proof by showing that $\operatorname{Stab}\left(\operatorname{Fix}\left(\operatorname{nc}_{c}(v)\right)\right)$ determines $\operatorname{cov}(v)$. Thus, if any of the maps in (i), (ii) or (iii) takes $v$ and $v^{\prime}$ to the same object, we have $\operatorname{cov}(v)=\operatorname{cov}\left(v^{\prime}\right)$ and thus $v=v^{\prime}$.

Specifically, we will show that for any $w \in W$ and any ordering $t_{1}, \ldots, t_{k}$ of $\operatorname{cov}(w)$, the stabilizer of the fixed set of $t_{1} \cdots t_{k}$ has canonical generators $\operatorname{cov}(w)$. Thus, in particular, since every parabolic subgroup has a uniquely defined set of canonical generators, $\operatorname{Stab}\left(\operatorname{Fix}\left(\operatorname{nc}_{c}(v)\right)\right)$ determines $\operatorname{cov}(v)$. 
Let $U=\bigcap_{t \in \operatorname{cov}(w)} H_{t}$. Lemma 2.27 states that $W^{\prime}:=\operatorname{Stab}(U)$ is a finite parabolic subgroup with canonical generators $\operatorname{cov}(w)$. For an ordering $t_{1}, \ldots, t_{k}$ of $\operatorname{cov}(w)$, for each $i \in[k]$ we have $t_{i}=w s_{i} w^{-1}$ for some $s_{i} \in S$. Let $J=$ $\left\{s_{1}, s_{2}, \ldots, s_{k}\right\}$. Then $W^{\prime}=w W_{J} w^{-1}$, so $W_{J}$ is finite. It is known (see [7, Theorem V.6.1] or [21, Lemma 3.16]) that for a finite Coxeter group $W^{\prime}$ with a reflection representation $V^{\prime}$ of dimension equal to the rank of $W^{\prime}$, a Coxeter element fixes no point of $\left(V^{\prime}\right)^{*}$ except the origin. Since $s_{1} \cdots s_{k}$ is a Coxeter element of $W_{J}$, we have $\operatorname{Fix}\left(s_{1} \cdots s_{k}\right)=\left(V_{J}\right)^{\perp}$, so $\operatorname{Fix}\left(t_{1} \cdots t_{k}\right)=w \operatorname{Fix}\left(s_{1} \cdots s_{k}\right)=w\left[\left(V_{J}\right)^{\perp}\right]=U$.

Remark 8.10. We cannot at present generalize [32, Theorem 6.1] to show that $\mathrm{nc}_{c}$ is a bijection from $c$-sortable elements of $W$ to $c$-noncrossing partitions, because it is not yet clear what is the right definition of noncrossing partitions in an infinite Coxeter group. (The "right" definition would be the definition that is most useful in studying Artin groups of infinite type.) The most obvious generalization is to lift the definition verbatim from the finite case, so that the $c$-noncrossing partitions are the elements of $[1, c]_{T}$. When $W$ is infinite, the map $\mathrm{nc}_{c}$ fails to be surjective onto $[1, c]_{T}$.

\section{The Cambrian fan}

In this section, we study the collection $\mathcal{F}_{c}$ consisting of all $c$-Cambrian cones together with all of their faces. We conjecture that this collection of cones is a fan in the usual sense (see Section 2.2) and prove the following weaker statement, whose terminology will be explained below.

Theorem 9.1. The collection $\mathcal{F}_{c}$ of cones is a fan in the interior of $\operatorname{Tits}(W)$. The decomposition, induced by $\mathcal{F}_{c}$, of $\operatorname{Tits}(W)$ into convex regions is a coarsening of the fan $\mathcal{F}(W)=\{w D: w \in W\}$

The first assertion of the theorem is made precise below. Informally, the assertion is that cones in $\mathcal{F}_{c}$ intersect nicely inside the Tits cone.

After proving Theorem 9.1, we gather some results (Propositions 9.4, 9.5 and 9.6) on the structure of the fan as it relates to standard parabolic subgroups and reflection functors (i.e. replacing $c$ by $s c s$ for $s$ initial). These results all follow from results of Sections 5 and 6 concerning $C_{c}$ and $\pi_{\downarrow}^{c}$. To conclude the section, we show that locally in the interior of $\operatorname{Tits}(W)$, the fan $\mathcal{F}_{c}$ looks like a Cambrian fan for a finite standard parabolic subgroup of $W$. For a very precise statement, see Theorem 9.8.

Let $\mathcal{F}_{c}$ be the collection of polyhedral cones that occur as the faces of the cones Cone $_{c}(v)$, as $v$ ranges over all of the $c$-sortable elements of $W$. Theorem 9.1 will imply in particular that when $W$ is finite, $\mathcal{F}_{c}$ is a fan in $V^{*}$. We conjecture that $\mathcal{F}_{c}$ is a fan even when $W$ is infinite. In Section 10 , we draw several examples of $\mathcal{F}_{c}$. In all of these examples (and many others), we have checked that $\mathcal{F}_{c}$ is in fact a fan. For the moment we optimistically refer to $\mathcal{F}_{c}$ as the $c$-Cambrian fan.

Showing that $\mathcal{F}_{c}$ is a fan in $V^{*}$ is difficult because we have no control over the intersections of cones outside $\operatorname{Tits}(W)$. In particular, we do not even know that the interiors of $\mathrm{Cone}_{c}(v)$ and $\mathrm{Cone}_{c}\left(v^{\prime}\right)$ are disjoint for $v \neq v^{\prime}$. However, in light of Theorem 6.3, we have great control of the intersections of cones inside $\operatorname{Tits}(W)$. The following definition is useful in describing the situation. 
Let $U$ be an open convex set in $\mathbb{R}^{n}$. We say that a collection $\mathcal{F}$ of cones in $\mathbb{R}^{n}$ is a fan in $U$ if

(i) for every $F \in \mathcal{F}$, the faces of $F$ are also in $\mathcal{F}$ and

(ii') for every subset $\mathcal{X} \subset \mathcal{F}$ and every $F \in \mathcal{X}$, there is a face $F^{\prime}$ of $F$ such that $\bigcap_{G \in \mathcal{X}} G \cap U=F^{\prime} \cap U$.

When $U=\mathbb{R}^{n}$, condition (ii') reduces to condition (ii) in the definition of a fan, given in Section 2.2.

The proof of Theorem 9.1 proceeds by first showing that the fan property only needs to be checked locally (Lemma 9.2, below) and then applying results of 30 . For any cone $F \in \mathbb{R}^{n}$ and any $x \in F$, the linearization $\operatorname{Lin}_{x}(F)$ of $F$ at $x$ is the set of vectors $x^{\prime} \in \mathbb{R}^{n}$ such that $x+\epsilon x^{\prime}$ is in $F$ for any sufficiently small nonnegative $\epsilon$. If $\mathcal{F}$ is a fan in $U$ and $x$ is any point in the union of the cones of $\mathcal{F}$, then the star of $x$ in $\mathcal{F}$ is the fan $\operatorname{Star}_{x}(\mathcal{F})=\left\{\operatorname{Lin}_{x}(F): x \in F \in \mathcal{F}\right\}$. For any cone $F$ of $\mathcal{F}$ such that $U$ intersects the relative interior of $F$, the star of $F$ in $\mathcal{F}$ is the fan $\operatorname{Star}_{F}(\mathcal{F})=\operatorname{Star}_{x}(\mathcal{F})$ for any $x$ in the intersection of $U$ with the relative interior of $F$. This is well-defined because $\mathcal{F}$ is a fan in $U$ : Condition (ii') implies that any cone in $\mathcal{F}$ that intersects $U \cap \operatorname{relint}(F)$ must contain $U \cap F$.

Lemma 9.2. Let $\mathcal{F}$ be a set of pointed polyhedral cones in $\mathbb{R}^{n}$ and let $U$ be an open convex set in $\mathbb{R}^{n}$. Suppose that $\mathcal{F}$ satisfies condition (i) of the definition of a fan. Suppose further that, for every $x \in U$, the set of cones $\operatorname{Lin}_{x}(\mathcal{F})=$ $\left\{\operatorname{Lin}_{x}(F): F \in \mathcal{F}\right\}$ is a fan. Then $\mathcal{F}$ is a fan in $U$.

Proof. We check condition (ii') of the definition. Fix a subset $\mathcal{X} \subseteq \mathcal{F}$ and a cone $F \in \mathcal{X}$ and set $I=\bigcap_{G \in \mathcal{X}} G$. We need to find a face $F^{\prime}$ of $F$ such that $I \cap U=F^{\prime} \cap U$. Notice that, since $F \in \mathcal{X}$, we know that $I \cap U \subseteq F$.

If $I \cap U$ is empty, then $U$ must not contain zero. In that case, we can take $F^{\prime}$ to be the face $\{0\}$. So, from now on, we assume that $I \cap U$ is nonempty.

Suppose that $\sigma$ and $\tau$ are two faces of $F$ whose relative interiors meet $I \cap U$. Let $s$ be a point in the intersection of $I \cap U$ with the relative interior of $\sigma$ and let $t$ be a point in the intersection of $I \cap U$ with the relative interior of $\tau$. Then, since $I \cap U$ is convex, $I \cap U$ contains the relative interior of the line segment st and thus meets the relative interior of the minimal face of $F$ containing $\sigma$ and $\tau$. Thus, there is a unique maximal face $F^{\prime}$ of $F$, among those faces of $F$ whose relative interior meets $I \cap U$. Moreover, $I \cap U \subseteq F^{\prime}$. Let $x_{0}$ be a point in the intersection of $I \cap U$ with the relative interior of $F^{\prime}$.

Suppose for the sake of contradiction that $F^{\prime} \cap U \neq I \cap U$. Let $x_{1}$ be a point in $F^{\prime} \cap U$ but not $I \cap U$. Consider the line segment $\overline{x_{0} x_{1}}$. By convexity, it lies in $F^{\prime} \cap U$, but the endpoint $x_{0}$ is in $I$ while the other endpoint $x_{1}$ is not. Since $I$ is closed and convex, $\overline{x_{0} x_{1}} \cap I=\overline{x_{0} x}$ for some point $x$ in the relative interior of the line segment $\overline{x_{0} x_{1}}$.

Now the cone $\bigcap_{G \in \mathcal{X}} \operatorname{Lin}_{x}(G)=\operatorname{Lin}_{x}(I)$ does not contain the vector $x_{1}-x$. However, $x$ is in the relative interior of $F^{\prime}$, so for any face $F^{\prime \prime}$ of $F$ containing $x$, we have $F^{\prime} \subseteq F^{\prime \prime}$ and, thus, $x_{1}-x \in \operatorname{Lin}_{x}\left(F^{\prime}\right) \subseteq \operatorname{Lin}_{x}\left(F^{\prime \prime}\right)$. So, $\bigcap_{G \in \mathcal{X}} \operatorname{Lin}_{x}(G)$ is not equal to any face of $\operatorname{Lin}_{x}(F)$, and thus $\left\{\operatorname{Lin}_{x}(F): F \in \mathcal{F}\right\}$ is not a fan. This contradiction shows that $F^{\prime} \cap U=I \cap U$.

Each cone $\operatorname{Cone}_{c}(v)$ of $\mathcal{F}_{c}$ is defined by the $n$ linear inequalities specified by the roots $C_{c}(v)$. Since the roots $C_{c}(v)$ are linearly independent, the cones $\operatorname{Cone}_{c}(v)$ are pointed. To check the local condition in Lemma 9.2 in the case where $\mathcal{F}$ is 
$\mathcal{F}_{c}$ and $U$ is the interior of $\operatorname{Tits}(W)$, we apply a result of 30. Consider for the moment the case where $W$ is finite, and temporarily relax the requirement that the reflection representation of $W$ have dimension equal to the rank of $W$. The Coxeter fan $\mathcal{F}(W)$ has a finite number of cones, and so the weak order on $W$ is a lattice. Recall that the elements of $W$ are in bijection with the maximal cones of $\mathcal{F}(W)$. For any lattice congruence $\Theta$ with congruence classes denoted $[w]_{\Theta}$, consider the collection of cones

$$
\operatorname{Cone}_{\Theta}(w)=\bigcup_{x \in[w]_{\Theta}} x D .
$$

A priori these cones may not be convex, but in fact they are convex as explained in [30, Section 5]. Let $\mathcal{F}_{\Theta}$ be the collection of all cones $\operatorname{Cone}_{\Theta}(w)$ for $w \in W$ together with their faces. The following theorem is a restatement of a very small part of 30 , Theorem 1.1].

Theorem 9.3. Let $W$ be finite and let $\Theta$ be any lattice congruence of the weak order on $W$. Then $\mathcal{F}_{\Theta}$ is a complete fan which coarsens $\mathcal{F}(W)$.

Here the term "complete" means that the union of the cones is all of $V^{*}$.

We now have all the necessary tools to prove the theorem.

Proof of Theorem 9.1, Let $x$ be in the interior of $\operatorname{Tits}(W)$ and let $W^{\prime}$ be the stabilizer of $x$ in $W$. By Lemma 2.27, $W^{\prime}$ is finite.

The fan $\operatorname{Star}_{x}(\mathcal{F}(W))$, the star of $x$ in the Coxeter fan of $W$, coincides with the Coxeter fan $\mathcal{F}\left(W^{\prime}\right)$ of $W^{\prime}$, where we again temporarily relax the requirement that the reflection representation of $W^{\prime}$ have dimension equal to the rank of $W^{\prime}$. The cones in $\operatorname{Star}_{x}(\mathcal{F}(W))$ are naturally in bijection with the cones $w D$ such that $x \in w D$. Let $w_{1}$ be such that $x \in w_{1} D$ and $w_{1} D$ is not separated from $D$ by any reflecting hyperplane in $W^{\prime}$ and let $w_{2}$ be such that $x \in w_{2} D$ and $w_{2} D$ is separated from $D$ by every reflecting hyperplane in $W^{\prime}$. Then a cone $w D$ contains $x$ if and only if $w$ is in the weak-order interval $\left[w_{1}, w_{2}\right]$. This interval is isomorphic to the weak order on $W^{\prime}$. Thus by Theorem 7.3 , the fibers of $\pi_{\downarrow}^{c}$ define a lattice congruence on the weak order on $W^{\prime}$. Theorem 9.3 implies that the fibers of $\pi_{\downarrow}^{c}$ define a fan which coarsens $\mathcal{F}\left(W^{\prime}\right)=\operatorname{Star}_{x}(\mathcal{F}(W))$. The maximal cones of this coarsening are the unions, over congruence classes, of the maximal cones of $\mathcal{F}\left(W^{\prime}\right)$. This fan is exactly the set $\left\{\operatorname{Lin}_{x}(F): x \in F \in \mathcal{F}_{c}\right\}$.

We now establish some important structural facts about the intersection of the $c$-Cambrian fan with $\operatorname{Tits}(W)$. For any collection $\mathcal{F}$ of cones, define $\mathcal{F} \cap \operatorname{Tits}(W)$ to be the set $\{F \cap \operatorname{Tits}(W): F \in \mathcal{F}\}$.

Proposition 9.4. Let $J \subseteq S$ and let $c^{\prime}$ be the restriction of $c$ to $W_{J}$. Then $\left[\left(P_{J}\right)^{-1}\left(\mathcal{F}_{c^{\prime}}\right)\right] \cap \operatorname{Tits}(W)$ is a coarsening of $\mathcal{F}_{c} \cap \operatorname{Tits}(W)$.

Less formally, restrict the representation of $W$ on $V$ to $W_{J}$ and build the $c^{\prime}$ Cambrian fan $\mathcal{F}_{c^{\prime}}$ in this representation of $W_{J}$. Then, within $\operatorname{Tits}(W)$, the walls of $\mathcal{F}_{c^{\prime}}$ are respected by the cones of $\mathcal{F}_{c}$. That is, each cone $\operatorname{Cone}_{c}(v)$ has $\operatorname{Cone}_{c}(v) \cap$ $\operatorname{Tits}(W) \subseteq$ Cone $_{c^{\prime}}\left(v^{\prime}\right)$ for some $c^{\prime}$-sortable element $v^{\prime}$ of $W_{J}$.

Proof. Let $v$ be a $c$-sortable element of $W$. We will show that $\operatorname{Cone}_{c}(v) \cap \operatorname{Tits}(W) \subseteq$ Cone $_{c^{\prime}}\left(v_{J}\right) \cap \operatorname{Tits}(W)$. Theorem 6.3 implies that

$$
\operatorname{Cone}_{c}(v) \cap \operatorname{Tits}(W)=\bigcup_{w \in\left(\pi_{\downarrow}^{c}\right)^{-1}(v)} w D
$$


for any $c$-sortable element $v$ of $W$. Thus

$$
P_{J}\left(\operatorname{Cone}_{c}(v) \cap \operatorname{Tits}(W)\right) \subseteq \bigcup_{w \in\left(\pi_{\downarrow}^{c}\right)^{-1}(v)} w_{J} D_{J} .
$$

By Proposition 6.13, the latter is contained in

$$
\bigcup_{x \in\left(\pi_{\downarrow}^{c^{\prime}}\right)^{-1}\left(v_{J}\right)} x D_{J}=\text { Cone }_{c^{\prime}}\left(v_{J}\right) \cap \operatorname{Tits}\left(W_{J}\right) .
$$

In the special case where $J$ is a singleton, the fan $\left(P_{J}\right)^{-1}\left(\mathcal{F}_{c^{\prime}}\right)$ has two cones, both halfspaces. In this case, Proposition 9.4 becomes the following.

Proposition 9.5. For any $s \in S$ and any c-sortable element $v$, the interior of $\operatorname{Cone}_{c}(v) \cap \operatorname{Tits}(W)$ is disjoint from $H_{s}$.

In other words, no $c$-Cambrian cone crosses the hyperplane $H_{s}$ within $\operatorname{Tits}(W)$. We conjecture that the interior of $\operatorname{Cone}_{c}(v)$ is disjoint from $H_{s}$, and in fact this conjecture is the only missing step in a proof, by the usual induction on length and rank, of the conjecture that $\mathcal{F}_{c}$ is a fan in $V$.

The recursive definition of $C_{c}(v)$ translates into the following geometric statement about the $c$-Cambrian fan. For any collection $\mathcal{F}$ of cones, define

$$
\mathcal{F}_{\geq s}=\left\{F \cap\left\{x^{*} \in V^{*}:\left\langle x^{*}, \alpha_{s}\right\rangle \leq 0\right\}: F \in \mathcal{F}\right\} .
$$

Thus, in $\mathcal{F}_{\geq s} \cap \operatorname{Tits}(W)$, each cone is intersected with the portion of $\operatorname{Tits}(W)$ consisting those of cones $w D$ for which $w \geq s$. Define $\mathcal{F}_{>s}$ by making the inequality in the definition strict, and define $\mathcal{F}_{\leq s}$ and $\mathcal{F}_{<s}$ by reversing the inequality.

Proposition 9.6. Let $s$ be initial in $c$. Then

(i) $\left(\mathcal{F}_{c}\right)_{\geq s} \cap \operatorname{Tits}(W)=s\left(\left(\mathcal{F}_{s c s}\right)_{\leq s}\right) \cap \operatorname{Tits}(W)$ and

(ii) $\quad\left(\mathcal{F}_{c}\right)_{<s} \cap \operatorname{Tits}(W)=\left[\left(P_{\langle s\rangle}\right)^{-1}\left(\mathcal{F}_{s c}\right)\right]_{<s} \cap \operatorname{Tits}(W)$.

The conjecture that $\mathrm{Cone}_{c}(v)$ is disjoint from $H_{s}$ for any $c$-sortable element $v$ would imply that both statements in the proposition remain true when " $\cap \operatorname{Tits}(W)$ " is removed throughout.

Proposition 5.3 is a combinatorial statement about $C_{c}(v)$. However, it can be understood geometrically in the following manner:

Proposition 9.7. Let s be final in c. Then $\left(\mathcal{F}_{c}\right)_{>s} \cap \operatorname{Tits}(W)=\left[\left(P_{\langle s\rangle}\right)^{-1}\left(\mathcal{F}_{c s}\right)\right]_{>s} \cap$ $\operatorname{Tits}(W)$.

See Section 10.1 for illustrations of Proposition 9.6 and 9.7

We conclude this section with a precise statement about the local structure of $\mathcal{F}_{c}$ inside $\operatorname{Tits}(W)$. Let $F$ be a cone in $\mathcal{F}_{c}$ whose relative interior intersects the interior of $\operatorname{Tits}(W)$. Let $x$ be any point in the intersection of the interior of $\operatorname{Tits}(W)$ with the relative interior of $F$. If $b$ is a vector in the interior of $D$, then as $\epsilon$ approaches zero from above, the point $x-\epsilon b$ remains in the interior of a certain $c$-Cambrian cone $\operatorname{Cone}_{c}(v)$, and also is in the interior of $\operatorname{Tits}(W)$. Since $\mathcal{F}_{c}$ is a fan in the interior of $\operatorname{Tits}(W)$, this $v$ is independent of the choice of $x$. For the same reason, the intersection of $F$ with the interior of $\operatorname{Tits}(W)$ equals the intersection of some collection of facets of $\mathrm{Cone}_{c}(v)$ with the interior of $\operatorname{Tits}(W)$. All of these facets of Cone $_{c}(v)$ are associated to cover reflections of $v$, and indeed, $F$ is specified uniquely by the choice of the $c$-sortable element $v$ and some subset of the cover reflections 
of $v$. Let $J$ be such that this subset of the cover reflections of $v$ is $\left\{v s v^{-1}: s \in J\right\}$. Call $v$ the c-sortable element above $F$ and call $J$ the set of simple generators through $F$. By Lemma 2.27, the group generated by the cover reflections of $v$ is finite. This group contains $v W_{J} v^{-1}$, so $W_{J}$ is finite and has a maximal element $w_{0}(J)$. Let $w=v w_{0}(J)$. We call $w$ the element below $F$. The element $w$ is characterized by the condition that $w D \cap F=v D \cap F$ and that $w D$ and $D$ are on the same side of the hyperplane $v H_{s}$ for each $s \in J$.

Let $v, w, F$ and $J$ be as in the previous paragraph. Since conjugation by $w_{0}(J)$ permutes $J$, we know that $w J w^{-1}=v w_{0}(J) J w_{0}(J)^{-1} v^{-1}$ is a subset of the cover reflections of $v$. Let $\operatorname{Cox}_{c}(F)$ or $\operatorname{Cox}_{c}(v, J)$ stand for the element $s_{1} s_{2} \cdots s_{k}$, where $J=\left\{s_{1}, s_{2}, \ldots, s_{k}\right\}$ and the elements of $J$ are numbered in the order that the cover reflections $w s_{i} w^{-1}$ appear in the reflection sequence for the $c$-sorting word of $v$. In particular, if $v J v^{-1}=\operatorname{cov}(v)$, then $\operatorname{Cox}_{c}(F)=w^{-1} \operatorname{nc}_{c}(v) w$. We can also describe $\operatorname{Cox}_{c}$ without any need to compute $c$-sorting words. In light of Proposition 3.11, if $\omega_{c}\left(\beta_{w s_{i} w^{-1}}, \beta_{w s_{j} w^{-1}}\right)>0$, then $s_{i}$ must come before $s_{j}$, and if $\omega_{c}\left(\beta_{w s_{i} w^{-1}}, \beta_{w s_{j} w^{-1}}\right)=0$, then $s_{i}$ and $s_{j}$ commute.

Theorem 9.8. If $F$ is a cone in $\mathcal{F}_{c}$ whose relative interior intersects the interior of $\operatorname{Tits}(W)$, then $\operatorname{Star}_{F}\left(\mathcal{F}_{c}\right)$ is combinatorially isomorphic to a finite Cambrian fan. Specifically, $\operatorname{Star}_{F}\left(\mathcal{F}_{c}\right)=w P_{J}^{-1}\left(\mathcal{F}_{\operatorname{Cox}_{c}(F)}\right)$, where $v$ is the c-sortable element above $F, J$ is the set of simple generators through $F$ and $w$ is the element below $F$.

This theorem generalizes [34, Proposition 8.12] and also strengthens the result by giving an explicit nonrecursive rule for computing $\operatorname{Cox}_{c}(F)$. The following lemma will be used in the proof.

Lemma 9.9. Let $s$ be initial in $c$, let $x$ be an element of $W$ such that $x \nsupseteq s$ but $s \vee x$ exists, and let $v$ be a c-sortable element of $W$ such that $v \nsupseteq s$ but $s \vee v$ exists. Then $\pi_{\downarrow}^{c}(x)=v$ if and only if $\pi_{\downarrow}^{s c s}(s \vee x)=s \vee v$.

Proof. In light of Proposition 2.20, the bijection of Theorem 7.4 restricts to a bijection between $c$-sortable elements $v$ with $v \leq x$ and scs-sortable elements $v^{\prime}$ with $s \leq v^{\prime} \leq s \vee x$. Corollary 6.2 now implies the lemma.

Proof of Theorem 9.8. Let $v, J$ and $w$ be as above. Write $c=s_{1} \cdots s_{n}$ and let $k$ be the smallest nonnegative integer $\leq n$ such that $s_{k}$ is not a cover reflection of $v$. If every $s_{i}$ is a cover reflection of $v$, then $W$ is finite, $v$ is the longest element of $W$ and $w$ is the identity, so that $\operatorname{Star}_{F}\left(\mathcal{F}_{c}\right)=\mathcal{F}_{c}$. Thus whenever $k$ is not well-defined, the theorem holds trivially. We argue by induction on the rank of $W$, on the length of $v$ (keeping $W$ fixed), and on $k$ (keeping $W$ and $v$ fixed).

The intersection of all cones $x D$ with $x \in[w, v]$ is a face $F^{\prime}$ of $\mathcal{F}$ with $F^{\prime} \subseteq F$ and $\operatorname{dim}\left(F^{\prime}\right)=\operatorname{dim}(F)$. For any point $p$ in the relative interior of $F^{\prime}$ we have $\operatorname{Star}_{F}\left(\mathcal{F}_{c}\right)=\operatorname{Star}_{p}\left(\mathcal{F}_{c}\right)$. Since $[w, v]=\{x \in W: p \in x D\}$, the $\operatorname{star}_{\operatorname{Star}}\left(\mathcal{F}_{c}\right)$ depends only on the equivalence relation induced on $[w, v]$ by the fibers of $\pi_{\downarrow}^{c}$. The map $z \mapsto w z$ is an isomorphism from the weak order on $W_{J}$ to the interval $[w, v]$. Thus the theorem is equivalent to the assertion that, for all pairs $x, y \in W_{J}$,

$$
\pi_{\downarrow}^{\operatorname{Cox}_{c}(v, J)}(x)=\pi_{\downarrow}^{\operatorname{Cox}_{c}(v, J)}(y) \text { if and only if } \pi_{\downarrow}^{c}(w x)=\pi_{\downarrow}^{c}(w y) .
$$

Let $s=s_{1}$. If $w \geq s$, then also $v \geq s$. The element $s v$ is $s c s$-sortable and $w^{-1} s w$ is not in $J$, so that the set $\left\{s v r v^{-1} s: r \in J\right\}$ is a subset of $\operatorname{cov}(s v)$. Thus $s v$ and $s w=s v w_{0}(J)$ are respectively the sortable element above and the element below 
some face of $\mathcal{F}_{s c s}$. Furthermore, $\operatorname{Cox}_{s c s}(s v, J)=\operatorname{Cox}_{c}(v, J)$, so the theorem follows in this case by induction on $\ell(v)$ and the recursive definition of $\pi_{\downarrow}^{c}$.

If $v \nsupseteq s$, then $v \in W_{\langle s\rangle}$, so $w \in W_{\langle s\rangle}$ and $J \subseteq\langle s\rangle$. The theorem follows in this case by induction on the rank of $W$.

If $v \geq s$ but $w \geq s$, then $s \in \operatorname{cov}(v)$ so that $k>1$. Furthermore, $v$ is also scs-sortable by Proposition 5.4(i), Proposition 2.30 and Theorem 7.1, Thus $(v, J)$ encodes a face in $\mathcal{F}_{s c s}$ and $w$ is the element below that face. Let $\operatorname{cov}(v)=$ $\left\{s, t_{2}, t_{3}, \ldots, t_{l}\right\}$. By Lemma [5.11] all of the $t_{i}$ lie in $W_{\langle s\rangle}$. Thus, for $2 \leq i, j \leq l$, we have $\omega_{c}\left(\beta_{t_{i}}, \beta_{t_{j}}\right)=\omega_{s c}\left(\beta_{t_{i}}, \beta_{t_{j}}\right)=\omega_{s c s}\left(\beta_{t_{i}}, \beta_{t_{j}}\right)$. Also, for $2 \leq i \leq l$, we have $\omega_{c}\left(\alpha_{s}, \beta_{t_{i}}\right) \geq 0$ and $\omega_{s c s}\left(\alpha_{s}, \beta_{t_{i}}\right) \leq 0$. So, setting $r=w^{-1} s w$, we know that $r$ is initial in $\operatorname{Cox}_{c}(v, J)$ and $\operatorname{Cox}_{s c s}(v, J)=r \operatorname{Cox}_{c}(v, J) r$.

If $x \geq r$ and $y \geq r$, then $w x \geq s$ and $w y \geq s$, so $\pi_{\downarrow}^{c}(w x)=\pi_{\downarrow}^{c}(w y)$ if and only if $\pi_{\downarrow}^{s c s}(s w x)=\pi_{\downarrow}^{s c s}(s w y)$. Since $s w x=w r x$ and $s w y=w r y$, the latter equation is $\pi_{\downarrow}^{s c s}(w r x)=\pi_{\downarrow}^{s c s}$ (wry) which by induction on $k$ holds if and only if $\pi_{\downarrow}^{\operatorname{Cox}_{s c s}(v, J)}(r x)=\pi_{\downarrow}^{\operatorname{Cox}_{s c s}(v, J)}(r y)$, or equivalently $\pi_{\downarrow}^{\operatorname{Cox}_{c}(v, J)}(x)=\pi_{\downarrow}^{\operatorname{Cox}_{c}(v, J)}(y)$.

If exactly one of $x$ and $y$ is above $r$, then exactly one of $w x$ and $w y$ is above $s$, and Proposition 6.9 implies that $\pi_{\downarrow}^{\operatorname{Cox}_{c}(v, J)}(x) \neq \pi_{\downarrow}^{\operatorname{Cox}_{c}(v, J)}(y)$ and $\pi_{\downarrow}^{c}(w x) \neq \pi_{\downarrow}^{c}(w y)$.

If $x \nsupseteq r$ and $y \geq r$, then $w x \nsupseteq s$ and $w y \nsupseteq s$, so Lemma 9.9 implies that $\pi_{\downarrow}^{c}(w x)=$ $\pi_{\downarrow}^{c}(w y)$ if and only if $\pi_{\downarrow}^{s c s}(s \vee(w x))=\pi_{\downarrow}^{s c s}(s \vee(w y))$. We have $s \vee w x=w(r \vee x)$ and $s \vee w y=w(r \vee y)$. Thus by induction on $k, \pi_{\downarrow}^{s c s}(s \vee(w x))=\pi_{\downarrow}^{s c s}(s \vee(w y))$ if and only if $\pi_{\downarrow}^{r \operatorname{Cox}_{c}(v, J) r}(r \vee x)=\pi_{\downarrow}^{r \operatorname{Cox}_{c}(v, J) r}(r \vee y)$. By Lemma 9.9 again, $\pi_{\downarrow}^{r \operatorname{Cox}_{c}(v, J) r}(r \vee x)=$ $\pi_{\downarrow}^{r \operatorname{Cox}_{c}(v, J) r}(r \vee y)$ if and only if $\pi_{\downarrow}^{\operatorname{Cox}_{c}(v, J)}(x)=\pi_{\downarrow}^{\operatorname{Cox}_{c}(v, J)}(y)$.

Remark 9.10. It is possible for a face $F$ of the Cambrian fan to lie in the boundary of the Tits cone. It seems plausible that Theorem 9.8 could be extended to describe this case. The primary challenge would be to generalize the definition of $\mathrm{Cox}_{c}$. In addition, the parabolic subgroup $W_{J}$ would have to be replaced by a generalized parabolic subgroup, so developing this theory would require developing a theory of generalized parabolic subgroups of rank other than 2. This has some subtleties, as explained in Remark 2.13 .

\section{RANK THREE EXAMPLES}

An irreducible Coxeter group $W$ of rank three acts as a reflection group on a two-dimensional manifold of constant curvature. Thus the rank three case is wellsuited to illustrations on the page. In this section, we illustrate many of the results of this paper using examples of rank three. We give examples of sortable elements and Cambrian fans in each of the three possible geometries: spherical, Euclidean and hyperbolic. As an aid to the reader scanning this section, the major concepts being illustrated at each point are written in boldface.

10.1. Type $\widetilde{G}_{2}$. The Coxeter groups with Euclidean geometry are the affine Coxeter groups (as well as some degenerate examples, such as the product of a finite group and an affine group). See e.g. [21, Sections 4.7, 6.5]. In rank three, the affine Coxeter groups are $\widetilde{A}_{2}, \widetilde{B}_{2}$ and $\widetilde{G}_{2}$. The Tits cone of an affine Coxeter group is an open halfspace plus one additional point (the origin) on the boundary. The action of the group on the Tits cone restricts to an action on a hyperplane parallel to the boundary of the halfspace. In this hyperplane, the group acts by Euclidean 


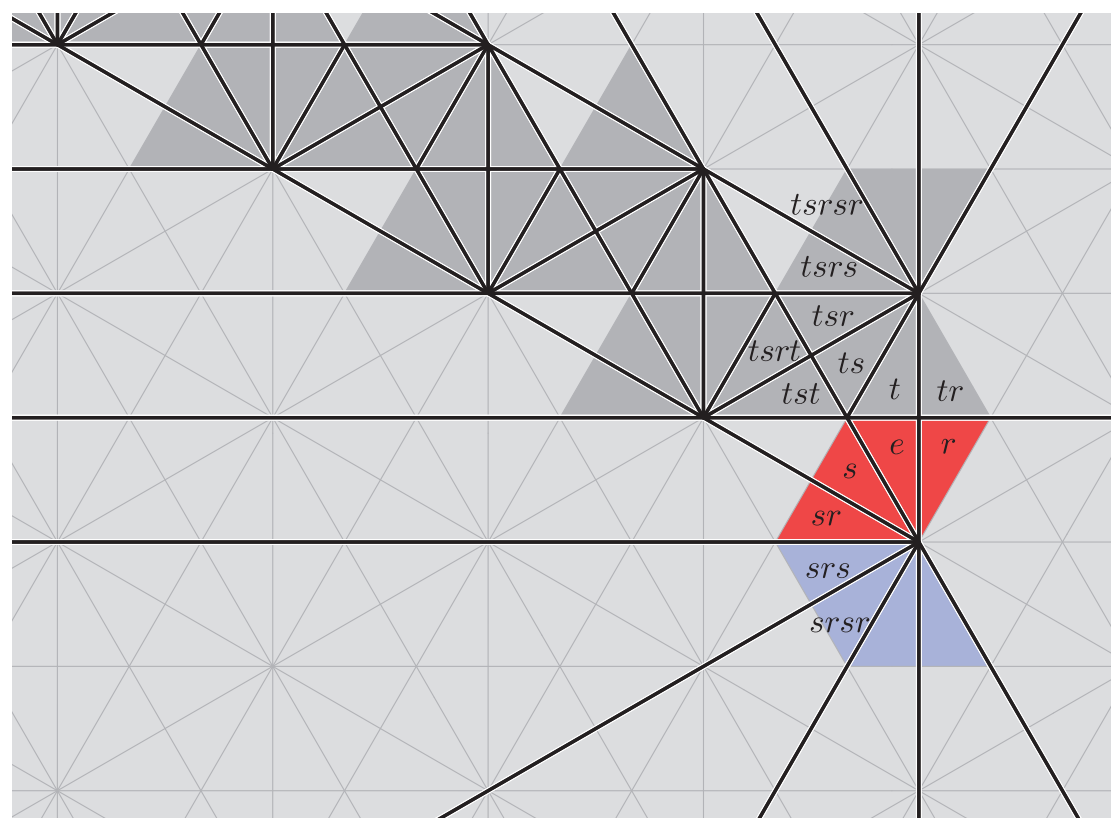

Figure 3. The Cambrian fan for $W$ of type $\widetilde{G}_{2}$ with $c=t s r$.

transformations. In particular, an affine Coxeter group of rank three is generated by reflections through certain lines in the plane.

The group of type $\widetilde{G}_{2}$ has simple generators $\{r, s, t\}$ with $m(r, s)=6, m(s, t)=3$ and $m(r, t)=2$. This is the symmetry group of the regular tiling of the plane by regular hexagons. Figures 3 and 4 show part of two Cambrian fans for $W$ of type $\widetilde{G}_{2}$. In Figure 3, the Coxeter element $c$ is $t s r$, while $c=s r t$ in Figure 4, The fine lines show the underlying Coxeter fan, and the bold lines indicate the Cambrian cones. The shaded triangles mark cones in the Coxeter fan corresponding to $c$-sortable elements. To the extent practical, the $c$-sortable elements are labeled with their $c$-sorting words.

To begin with, we focus on the shaded triangles in order to visualize results about sortable elements. The colors of the shaded triangles help to illustrate Theorem 7.4. Notice that $t$ is initial in $c=t s r$. In Figure 3, the gray shaded triangles are $c$-sortable elements above $t$ in the weak order. The red (or darker gray) shaded triangles are $c$-sortable elements $v$ not above $t$ such that $t \vee v$ exists. The blue (or lighter gray) shaded triangles are $c$-sortable elements $v$ such that $t \vee v$ does not exist. The bijection of Theorem 7.4 maps the gray and red triangles in Figure 3 to the full set of triangles in Figure 4 . The gray triangles are reflected through the horizontal line representing $H_{t}$, while each red triangle representing $v$ is mapped to $t \vee v$.

One can also see the canonical join representations of $c$-sortable elements in these figures. For example, Proposition $\mathbf{8 . 2}$ is illustrated in Figure 4, where the $s r t$-sortable element srtsrsrs is canonically the join of $s r t$-sortable elements $s r$ 


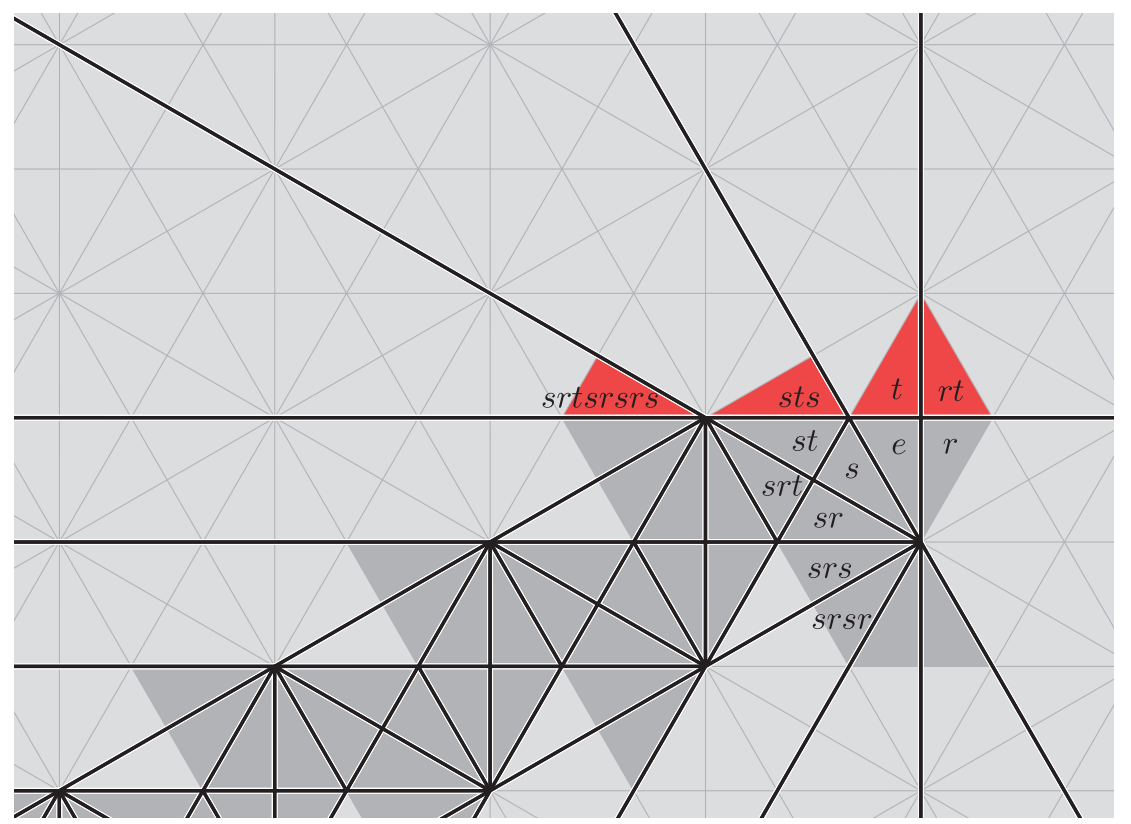

Figure 4. The Cambrian fan for $W$ of type $\widetilde{G}_{2}$ with $c=$ srt.

and $t$. This picture also illustrates the proof of Theorem $\mathbf{8 . 1}$ The element $s r$ is the unique smallest element below srtsrsrs having the reflection srs as an inversion, and $t$ is the unique smallest element below srtsrsrs having the reflection $t$ as an inversion. Theorem 8.3 manifests itself in these pictures as the fact that, for each bold line segment in the picture, there is a unique minimal sortable element $j$ of $W$ that is above the corresponding hyperplane $H$. This element $j$ is join-irreducible, that is, exactly one of the edges of its triangle $j D$ separates $j D$ from a triangle closer to $D$. This edge of $j D$ is contained in $H$, and $j$ is the only join-irreducible element such that $j D$ has an edge contained in $H$ that separates $j D$ from $D$.

We now consider the Cambrian cones in the figures. Theorem 7.4 is a statement about sortable elements, and Proposition 9.6 is the corresponding statement about Cambrian fans. In accordance with Proposition 9.6(i), since $t$ is initial in $t s r$, the partial fans $\left(\mathcal{F}_{t s r}\right)_{\geq t} \cap \operatorname{Tits}(W)$ and $\left(\mathcal{F}_{s r t}\right)_{\leq t} \cap \operatorname{Tits}(W)$ are related by reflection through $H_{t}$. The partial fan $\left(\mathcal{F}_{t s r}\right)_{<t}$ agrees with the $s r$-Cambrian fan in $W_{\langle t\rangle}=W_{\{r, s\}}$ in accordance with Proposition 9.6(ii). Proposition 9.4 specializes to the following statement about these pictures: For each vertex $x$ of the shaded triangle representing the identity element $e$, the bold line segments emanating from $x$ extend indefinitely, so that no Cambrian cone crosses them. In particular (Proposition 9.5), the line segments bounding the identity element extend indefinitely.

Figures 3 and 4 also illustrate our main lemmas regarding forced and unforced skips: Propositions 5.1, 5.2, 5.3 and 5.4. All of these, due to Theorem 6.3, can be interpreted as assertions about the Cambrian cone $\mathbf{C o n e}_{\boldsymbol{c}}(\boldsymbol{v})$. Proposition 5.2 states that the lower walls (the walls separating the cone from the dominant chamber $D$ ) of each Cambrian cone $\operatorname{Cone}_{c}(v)$ correspond to the cover reflections of $v$. These cover reflections appear as the lower walls of the triangle corresponding to $v$. 
Similarly, with a bit of calculation, we observe that the upper walls are given by unforced skips in the $c$-sorting word. Consider for example the Cambrian cone Cone $_{\text {srt }}($ srtsrsrs). Its lower walls are given by its cover reflections $t$ and $s r s$. The letter $t$ is skipped in the word srtsr.srs (as indicated by the dot in the word). This skip is unforced, with corresponding reflection srtsrtrstrs $=$ srsrs, so the hyperplane $H_{\text {srsrs }}$ defines the upper wall of $\mathrm{Cone}_{\text {srt }}($ srtsrsrs). In Figure 4 , the hyperplane $H_{\text {srsrs }}$ is below, and parallel to, the hyperplane $H_{t}$, reflecting the fact that these two hyperplanes do not intersect within the Tits cone but rather intersect on the boundary of the Tits cone. Within the Tits cone, Cone srt $_{\text {(srtsrsrs) is }}$ bounded by $H_{t}$ and $H_{s r s}$, while, in the negation of the Tits cone, Cone ${ }_{s r t}$ (srtsrsrs) is bounded by $H_{\text {srsrs }}$ and $H_{\text {srs }}$. The remainder of the cone Cone ${ }_{\text {srt }}$ (srtsrsrs) is pictured below in Figures 5 and 6 .

The geometric content of Proposition 5.3 appears explicitly as Proposition 9.7. This geometry is apparent, for example, in Cone $t s r(t r)$ in Figure 3 . One wall of Cone $_{t s r}(t r)$ is given by the hyperplane $H_{r}$, and the other walls are obtained by extending the walls of $\mathrm{Cone}_{t s}\left((t r)_{\langle r\rangle}\right)=\mathrm{Cone}_{t s}(t)$. Similarly, Proposition 5.4 is exemplified by $\mathrm{Cone}_{t s r}(t s t)$ in Figure 3 One lower wall of Cone ${ }_{t s r}(t s t)$ is the hyperplane $H_{t}$. The other lower wall is obtained by extending the lower wall of Cone $_{s r}\left((t s t)_{\langle t\rangle}\right)=\mathrm{Cone}_{s r}(s)$, while the upper wall is obtained by reflecting the upper wall of Cone ${ }_{s r}\left((t s t)_{\langle t\rangle}\right)$ across $H_{t}$.

We now point out how the content of Theorem $\mathbf{9 . 8}$ can be seen in these pictures. Consider in Figure 4 the two-dimensional cone $F$ represented in this planar slice by the line segment separating Cone $_{\text {srt }}($ sts $)$ from Cone srt $_{\text {(srtsrsrs). Taking any } x \text { in }}$ the relative interior of this cone and moving a short distance away from the interior

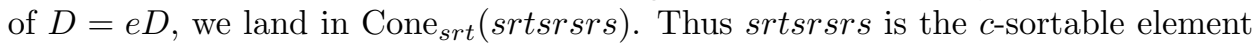
above $F$. The face $F$ of Cone $_{\text {srt }}$ (srtsrsrs) is the intersection of Cone srt $_{\text {srtsrsrs) }}$ with $H_{\text {srs }}$, and srs is a cover reflection srtsrsrs.r.srsrstrs of srtsrsrs. Thus $\{r\}$ is the set of simple generators through $F$, so $w_{0}(\{r\})=r$ and srtsrsrs.r $=$ stsrsrs is the element below $F$. (The cone stsrsrs $D$ is not labeled, but it is immediately adjacent to srtsrsrs $D$ through the face $F$.) The assertion of Theorem 9.8 is that the star of $F$ in $\mathcal{F}_{\text {srt }}$ is stsrsrs $P_{\{r\}}^{-1}\left(\mathcal{F}_{r}\right)$. The fan $\mathcal{F}_{r}$ consists of the origin and two opposite rays inside the one-dimensional space $V_{\{r\}}^{*}$, and $P_{\{r\}}^{-1}\left(\mathcal{F}_{r}\right)$ in $V^{*}$ consists of the hyperplane $H_{r}$ and the two halfspaces it defines. Thus $\operatorname{Star}_{F}\left(\mathcal{F}_{s r t}\right)$ consists of the hyperplane stsrsrs $H_{r}=H_{\text {srs }}$ and the two halfspaces it defines.

As another example, now take $F$ to be the ray defined as the intersection of Cone $_{\text {srt }}$ (srtsrsrs) with $H_{\text {srs }}$ and $H_{t}$. Note that $t=$ srtsrsrs.s.srsrstrs is the other cover reflection of srtsrsrs. Again srtsrsrs is the $c$-sortable element above $F$, and now $\{r, s\}$ is the set of simple generators through $F$, with $w_{0}(\{r, s\})=s r s r s r$. The element below $F$ is srtsrsrs.srsrsr $=$ st. The reflection sequence of srtsrsrs is

$$
s, \text { srs, sts, srtstrs, srtsrstrs, stsrsrsts, tsrst, } t \text {, }
$$

and in particular srs occurs before $t$. Thus $\operatorname{Cox}_{s r t}(F)$ is $r s$, rather than $s r$. The assertion of Theorem 9.8 can be stated as follows in the context of Figure 4 Take the lower vertex of the triangle representing $D$ as an origin for the plane and build the $r s$-Cambrian fan around this origin. (Our Euclidean plane has no natural origin; the plane in which our pictures are drawn does not contain the origin of $V^{*}$.) Then the rotation in the plane corresponding to st maps the $r s$-Cambrian fan to the star of $F$. 


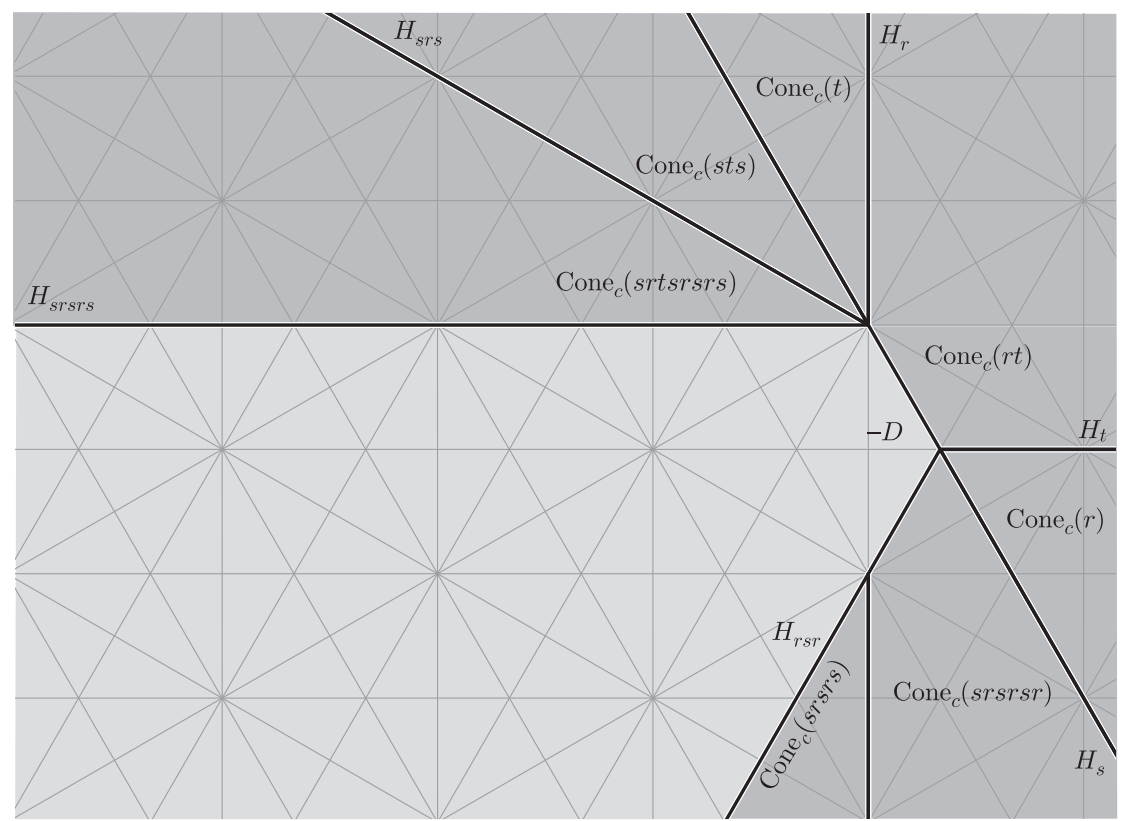

Figure 5. The Cambrian fan as it intersects the negative Tits cone, for $W$ of type $\widetilde{G}_{2}$ with $c=$ srt.

We now offer some additional illustrations of the srt-Cambrian fan for $W$ of type $\widetilde{G}_{2}$ which illustrate how the Cambrian fan extends beyond the Tits cone. First, we show the intersection of the srt-Cambrian fan with the negative Tits cone $-\operatorname{Tits}(W)$. To interpret this picture, recall that Figure 4 shows the intersection of the srt-Cambrian fan with a plane in $\operatorname{Tits}(W)$. We are looking at that plane from far above (i.e. far into $\operatorname{Tits}(W)$ ). Figure 5 shows, from the same vantage point, the intersection of the srt-Cambrian fan with a parallel plane in $-\operatorname{Tits}(W)$. The fan is shaded gray, and its maximal cones are labeled, as are the reflecting hyperplanes bounding these cones. The antipodal dominant chamber $-D$, located outside the Cambrian fan, is also labeled.

To unify these two views, Figure 6] shows the Cambrian fan in stereographic projection. That is, the intersection of the Cambrian fan with the unit sphere in $V^{*}$ defines a complex of spherical simplices. Figure [6 shows this simplicial complex in stereographic projection. The point at infinity is the image of a point in the antipodal dominant chamber $-D$. The boundary of the Tits cone projects to the gray circle. The three walls of Cone $_{\text {srt }}$ (strsrsrs) are now visible. (The label srtsrsrs has been replaced by a black dot.)

The form $\omega_{c}$ is a key ingredient in the proofs presented in this paper. However, the precise value of $\omega_{c}$ is not important, while the sign of $\omega_{c}$ is decisive. There is an elegant way to visualize the sign of $\omega_{c}$ for Coxeter groups of rank 3. Every skew symmetric form $\omega$ on $\mathbb{R}^{3}$ is of the form $\omega(\alpha, \beta)=\operatorname{Vol}(\alpha \wedge \beta \wedge \zeta)$ for some $\zeta \in \mathbb{R}^{3}$, where $\mathrm{Vol}$ is the volume form. Then $\omega(\alpha, \beta)$ is positive or negative according 


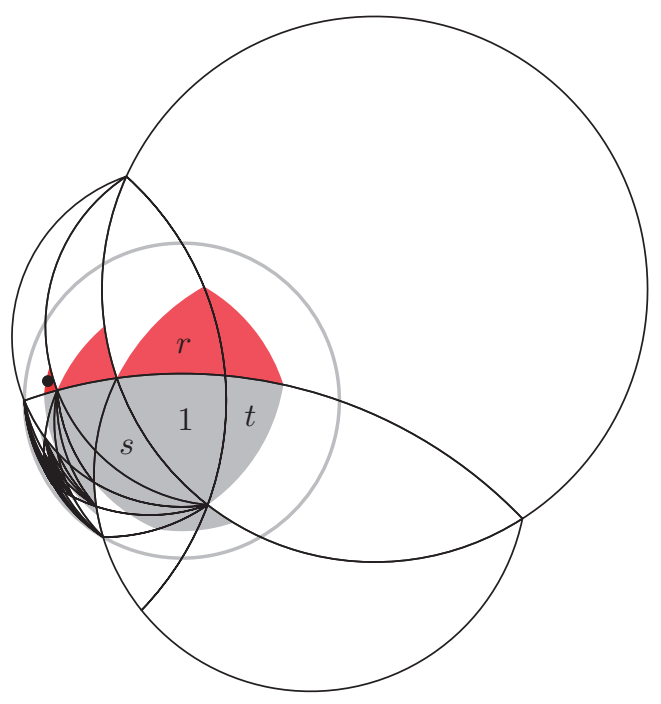

FiguRE 6. A stereographic view of the Cambrian fan for $W$ of type $\widetilde{G}_{2}$ with $c=$ srt.

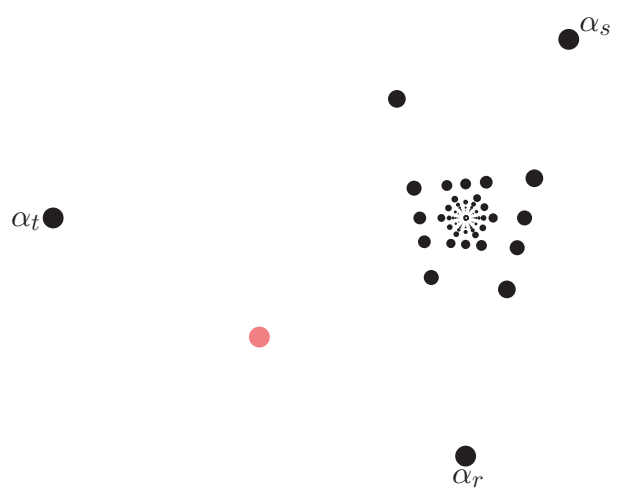

Figure 7 . The $\widetilde{G}_{2}$ root system with $\zeta_{t s r}$.

to whether the angle from $\alpha$ to $\beta$ circles the line spanned by $\zeta$ in a clockwise or counterclockwise manner. For a rank 3 Coxeter group, let $\zeta_{c}$ be such that $\omega_{c}(\alpha, \beta)=\operatorname{Vol}\left(\alpha \wedge \beta \wedge \zeta_{c}\right)$. From $\omega_{c}(\alpha, \beta)=\omega_{c}(c \alpha, c \beta)$ (and the fact that $c$ acts by a matrix of determinant -1 in rank 3$)$ we deduce that $\zeta_{c}$ is a $(-1)$-eigenvector of $c$. This makes it easy to compute $\zeta_{c}$. Figure 7 illustrates the example where $W$ is of type $\widetilde{G}_{2}$ and $c=t s r$. Each positive root generates a ray, and the vector $\zeta_{c}$ generates a ray. The plane of the picture is a plane that intersects all of these rays, and each ray is represented by its intersection with the plane. Since there are infinitely many roots, they are shown as black dots whose size becomes smaller as the simple-root coordinates of the roots increase. The ray for $\zeta_{c}$ is shown in red (or gray). In this planar picture, $\omega_{c}\left(\beta, \beta^{\prime}\right)>0$ if the angle $\beta \zeta_{c} \beta^{\prime}$ (projected to the plane) is clockwise. In Section 10.3, a similar example will be taken a step further to illustrate Proposition 3.11 . 
10.2. Type $B_{3}$. When $W$ is finite, the Tits cone $\operatorname{Tits}(W)$ is all of $V^{*}$. Thus the Cambrian fan also occupies all of $V^{*}$. In particular, intersecting with the unit sphere, the Cambrian fan defines a simplicial sphere. Figure 8 shows a Cambrian fan (as a simplicial sphere in stereographic projection) in the case where $W$ is of type $B_{3}$. Here $W$ is generated by $\{r, s, t\}$ with $m(r, s)=4, m(s, t)=3$ and $m(r, t)=2$, and the figure shows the case where $c=r s t$. The stereographic projection, despite being difficult to interpret at first, has the advantage of showing essentially the entire fan in one picture. The three largest circles represent the simple generators, with $t$ being the largest, then followed by $r$, and then by $s$. The dominant chamber $D$, representing the identity element, is the triangle inside all three of these circles. As in previous figures, the sortable elements are represented by shaded regions. The unbounded region represents the longest element $w_{0}$.

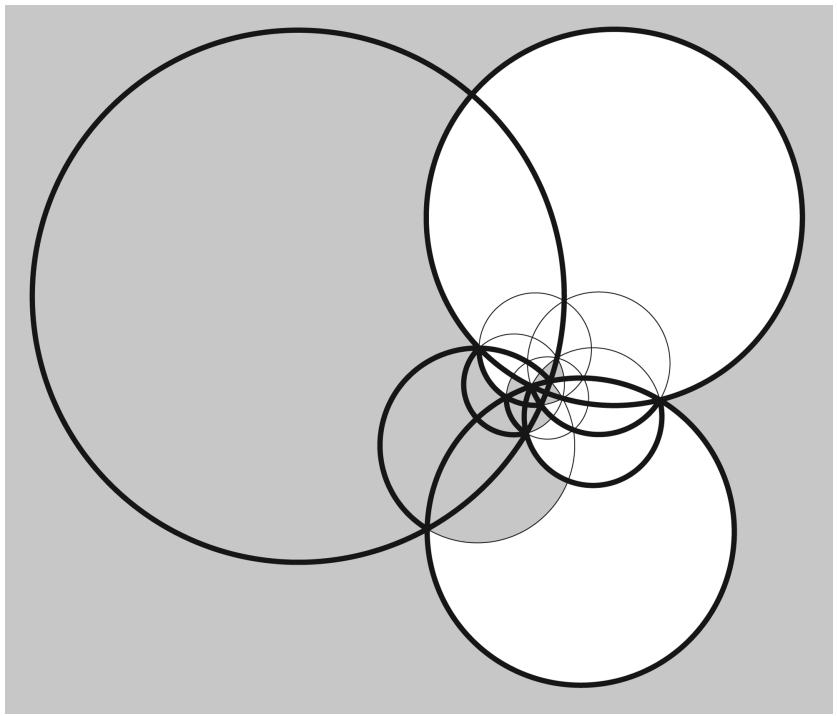

Figure 8. A stereographic view of the Cambrian fan for $W$ of type $B_{2}$ with $c=$ rst.

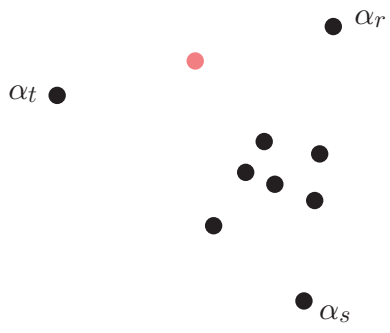

Figure 9. The $B_{3}$ root system, with $\zeta_{\text {rst }}$.

The Cambrian fan shown in Figure 8 is isomorphic, as a simplicial complex, to the dual of the generalized associahedron of type $B_{3}$. (See [16.) More generally, 


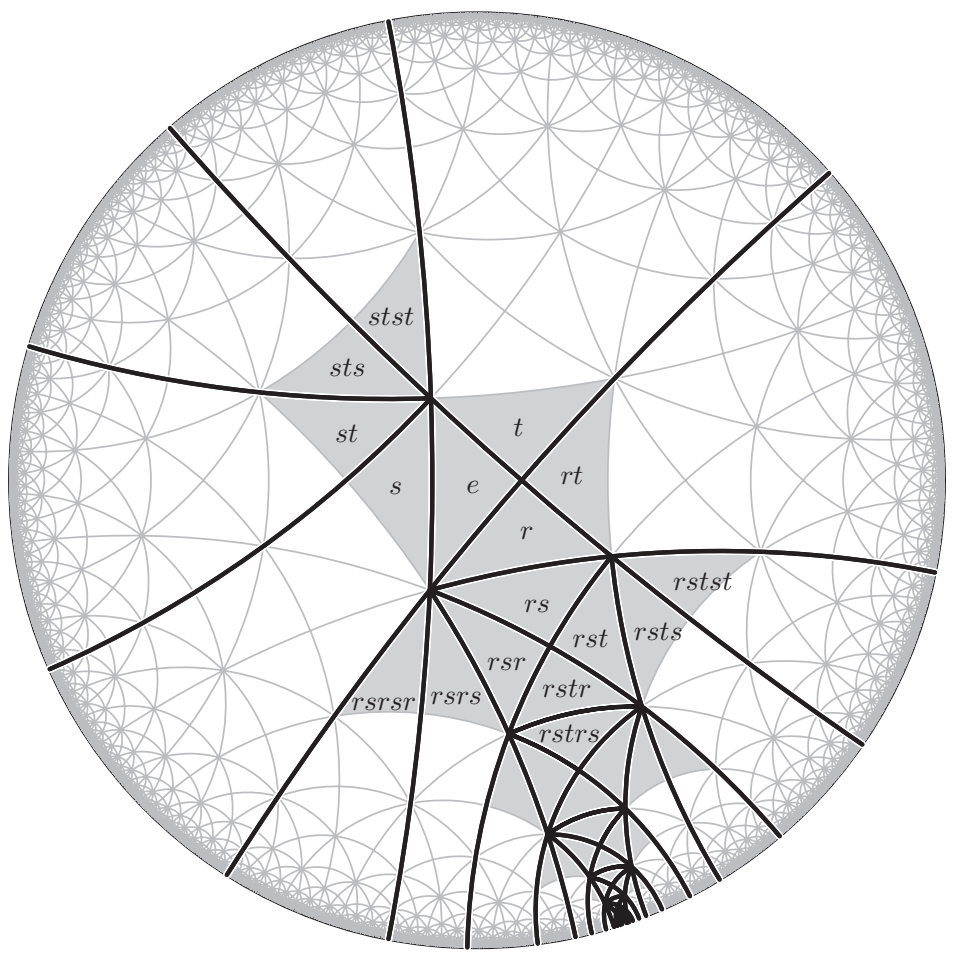

Figure 10. A Cambrian fan in the Poincaré disk.

as proved in [34, for any finite Coxeter group and any Coxeter element $c$, the $c$ Cambrian fan is isomorphic to the normal fan of the generalized associahedron of the appropriate type.

Figure 9 illustrates $\omega_{\text {rst }}$ for $W$ of type $B_{3}$ and $c=$ rst. As in Figure 7 , each represents a root, except for the red dot, which represents $\zeta_{c}$. Again $\omega_{c}\left(\beta, \beta^{\prime}\right)>0$ if the angle $\beta \zeta_{c} \beta^{\prime}$ is clockwise.

10.3. A hyperbolic example. Whenever a rank three Coxeter group is neither finite nor affine, it is always possible to choose a reflection representation of $W$ such that $\operatorname{Tits}(W)$ is a circular cone. The reflecting hyperplanes, intersected with one sheet of an appropriate hyperboloid, are lines in the hyperboloid model of the hyperbolic plane. These lines can further be projected to a disk so as to become lines in the Poincaré model of the hyperbolic plane. In particular, the intersection of the Cambrian fan with $\operatorname{Tits}(W)$ defines a decomposition of the Poincaré disk into convex regions. Figure 10 shows this decomposition in the case where $W$ is generated by $\{r, s, t\}$ with $m(r, s)=5, m(s, t)=4$ and $m(r, t)=2$. The Coxeter element is $c=r s t$.

As in the affine example of $\widetilde{G}_{2}$, the Cambrian fan in this example extends beyond the Tits cone. Figure 11 shows the stereographic projection of the Cambrian fan. (Some parts of $-D$ and nearby regions have been cropped to make the image fit on the page.) A representative group of reflecting hyperplanes (also extending outside $\operatorname{Tits}(W)$ ) is also shown. These reflecting hyperplanes also serve to define the boundaries of $\operatorname{Tits}(W)$ and $-\operatorname{Tits}(W)$ : The area outside of $\operatorname{Tits}(W)$ and 


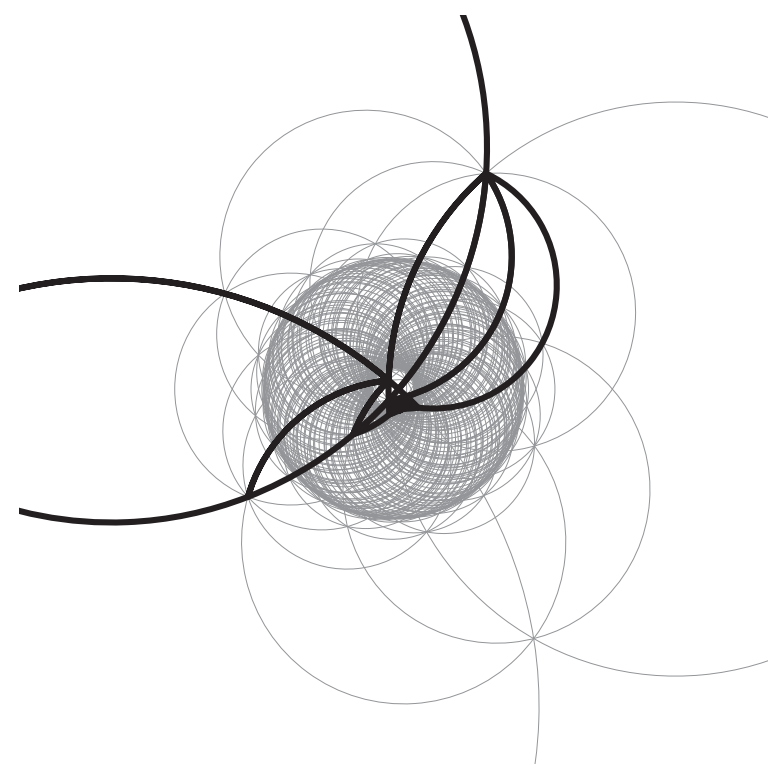

FiguRE 11. A stereographic view of a hyperbolic Cambrian fan.

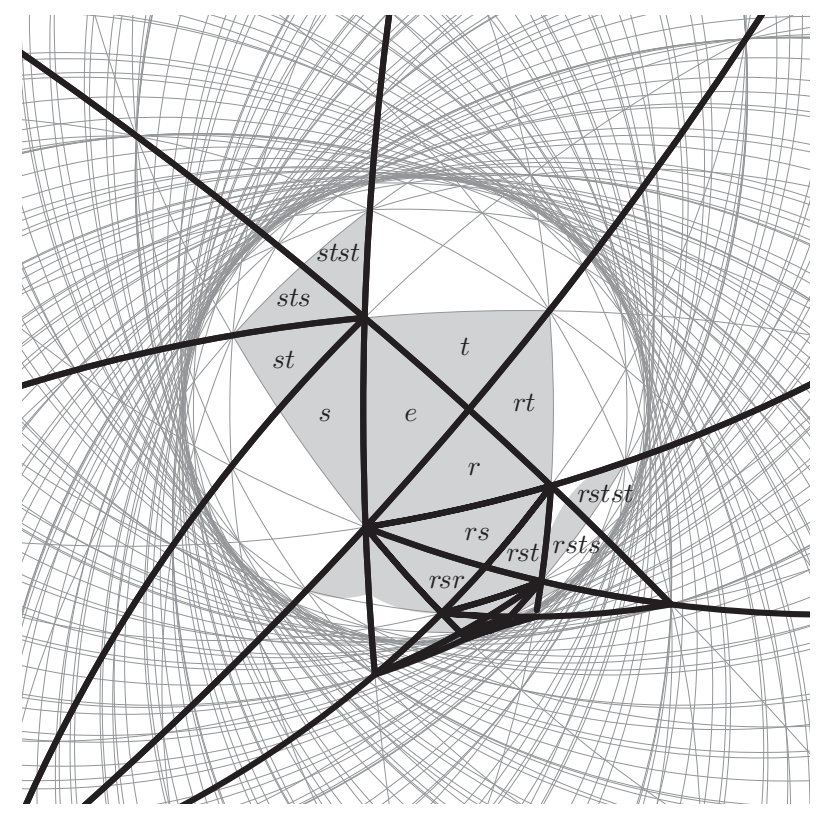

Figure 12. A magnified view of the fan from Figure 11 .

$-\operatorname{Tits}(W)$ is dense with reflecting hyperplanes. Figure 12 is a magnified view of the neighborhood of $\operatorname{Tits}(W)$. 
$\alpha_{r}(1)$

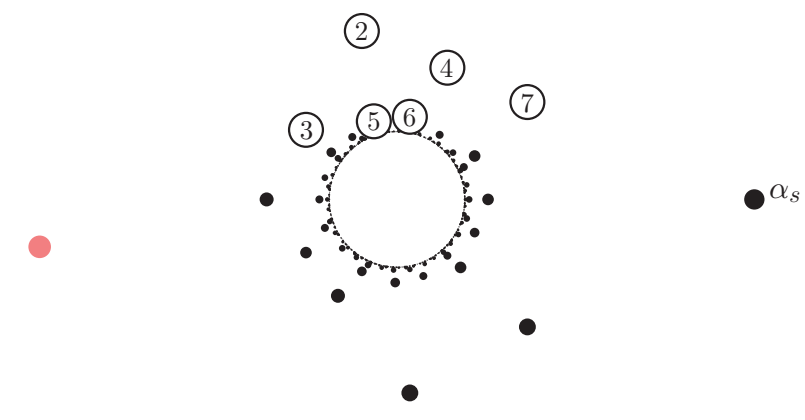

FiguRE 13. The reflection sequence of an $r$ st-sorting word.

Figure 13 illustrates the root system for this hyperbolic Coxeter group. The infinitely many roots are projected to the page in a manner similar to the $\widetilde{G}_{2}$ example in Section 10.1, except that certain roots are represented by circled numbers, for reasons we will explain below. The small circle containing no dots is the dual cone in $V$ to $\operatorname{Tits}(W) \subset V^{*}$. Also shown is $\zeta_{r s t}$. Once again, $\omega_{r s t}\left(\beta, \beta^{\prime}\right)>0$ if the angle $\beta \zeta_{c} \beta^{\prime}$ is clockwise.

The example of Figure 13 can be taken further to provide an illustration of Proposition 3.11. Figure 14] is a representation of the rst-sorting word rstrsts which represents an $r s t$-sortable element. A word in the generators $S$ of $W$ describes a walk through the maximal cones in $\operatorname{Tits}(W)$, starting at the cone $D$ representing the identity. In Figure 14, the cones visited on this walk are numbered, starting with 0 on the identity element and ending with 7 on the shaded triangle representing rstrsts. Each number $i$ labels the element given by the first $i$ letters of the word rstrsts. Each of these elements is rst-sortable.

Recall that a reduced word $a_{1} \cdots a_{k}$ in the generators $S$ defines a reflection sequence $t_{1}, \ldots, t_{k}$ with $t_{i}=a_{1} \cdots a_{i} \cdots a_{1}$. The circled numbers in Figure 13 represent the reflection sequence associated to rstrsts (as a sequence of positive roots). For example, the root labeled 1 is associated to the reflection $r$, the root labeled 2 is associated to the reflection $r s r$, and so forth. Proposition 3.11 asserts that this reflection sequence must have $\omega_{c}\left(\beta_{t_{i}}, \beta_{t_{j}}\right) \geq 0$ for all $i \leq j$, with strict inequality holding unless $t_{i}$ and $t_{j}$ commute. Indeed, the labeling of the roots in Figure 13 is consistent with the order in which these roots are met by a clockwise line through $\zeta_{r s t}$, initially vertical. The roots marked 3 and 4 are collinear with $\zeta_{r s t}$, reflecting the fact that the reflections $t_{3}$ and $t_{4}$ in the reflection sequence commute. 


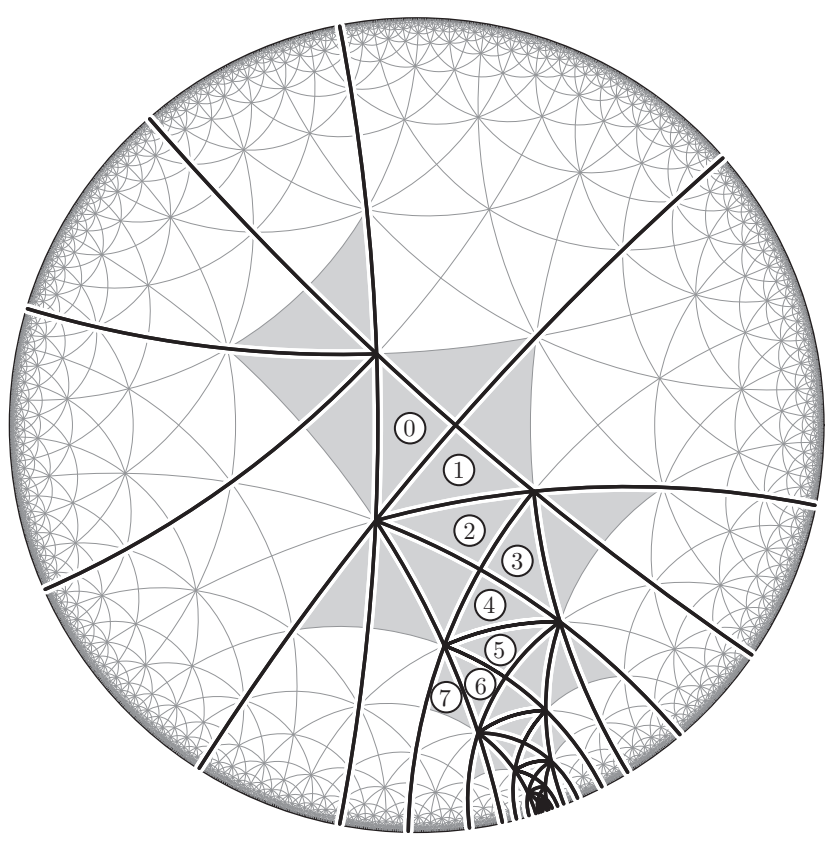

FiguRE 14. An rst-sorting word.

The word rsrtsts, obtained from rstrsts by transposing the adjacent commuting letters $r$ and $t$, also satisfies condition (i) of Proposition 3.11.

The circled numbers are precisely the roots corresponding to inversions of $r$ strsts. By viewing them as a set, while ignoring the ordering, we can see that rstrsts is $r$ st-aligned.

\section{ACKNOWLEDGMENTS}

We are grateful to Matthew Dyer, John Stembridge and Hugh Thomas for helpful conversations. We are also grateful to anonymous referees for helpful comments.

\section{REFERENCES}

[1] N. A'Campo, Sur les valeurs propres de la transformation de Coxeter. Invent. Math. 33 (1976), no. 1, 61-67. MR0424967 (54:12925)

[2] I. Assem, D. Simson and A. Skowroński, Elements of the Representation Theory of Associative Algebras, Volume I. London Mathematical Society Student Texts, 65, Cambridge University Press, Cambridge, UK, 2006. MR2197389 (2006j:16020)

[3] I. Bernšteľn, I. Gel'fand, and V. Ponomarev, Coxeter functors, and Gabriel's theorem. Uspehi Mat. Nauk 28 (1973) no. 2 (170), 19-33. English translation in Russian Math. Surveys 28 (1973), no. 2, 17-32. MR0393065 (52:13876)

[4] D. Bessis, The dual braid monoid. Ann. Sci. Ecole Norm. Sup. 36 (2003), 647-683. MR.2032983 (2004m:20071)

[5] S. Billey and T. Braden, Lower bounds for Kazhdan-Lusztig polynomials from patterns. Transform. Groups 8 (2003), no. 4, 321-332. MR2015254(2005a:20060)

[6] A. Björner and F. Brenti, Combinatorics of Coxeter groups. Graduate Texts in Mathematics, 231, Springer, New York, 2005. MR2133266 (2006d:05001) 
[7] N. Bourbaki, Lie groups and Lie algebras. Chapters 4-6. Translated from the 1968 French original by Andrew Pressley. Elements of Mathematics. Springer-Verlag, Berlin, 2002. MR.1890629 (2003a:17001)

[8] T. Brady and C. Watt, $K(\pi, 1)$ 's for Artin groups of finite type. Proceedings of the Conference on Geometric and Combinatorial Group Theory, Part I (Haifa, 2000). Geom. Dedicata 94 (2002), 225-250. MR.1950880 (2004i:20066)

[9] T. Brady and C. Watt, A partial order on the orthogonal group. Comm. Algebra 30 (2002), no. 8, 3749-3754. MR.1922309 (2003h:20083)

[10] N. Caspard, The lattice of permutations is bounded. Internat. J. Algebra Comput. 10 (2000), no. 4, 481-489. MR.1776052 (2001d:06008)

[11] N. Caspard, C. Le Conte de Poly-Barbut and M. Morvan, Cayley lattices of finite Coxeter groups are bounded. Adv. in Appl. Math. 33 (2004), no. 1, 71-94. MR2064358(2005b:06006)

[12] V. Deodhar, A note on subgroups generated by reflections in Coxeter groups. Arch. Math. (Basel) 53 (1989), no. 6, 543-546. MR.1023969 (91c:20063)

[13] H. Derksen and J. Weyman, Quiver representations. Notices Amer. Math. Soc. 52 (2005), no. 2, 200-206. MR2110070 (2005k:16033)

[14] M. Dyer, Hecke algebras and shellings of Bruhat intervals. Compositio Math. 89 (1993), no. 1, 91-115. MR:1248893 (95c:20053)

[15] M. Dyer, Reflection subgroups of Coxeter systems. J. Algebra 135 (1990), no. 1, 57-73. MR:1076077 (91j:20100)

[16] S. Fomin and A. Zelevinsky, $Y$-systems and generalized associahedra. Ann. of Math. (2) 158 (2003), no. 3, 977-1018. MR2031858 (2004m:17010)

[17] S. Fomin and A. Zelevinsky, Cluster algebras II: Finite type classification. Inventiones Mathematicae 154 (2003), 63-121. MR2004457 (2004m:17011)

[18] S. Fomin and A. Zelevinsky, Cluster algebras IV: Coefficients. Compositio Mathematica 143 (2007), 112-164. MR2295199 (2008d:16049)

[19] R. Freese, J. Ježek, and J. Nation, Free lattices. Mathematical Surveys and Monographs, 42. American Mathematical Society, Providence, RI, 1995. MR1319815 (96c:06013)

[20] R. Howlett, Coxeter groups and M-matrices. Bull. London Math. Soc. 14 (1982), no. 2, 137-141. MR647197 (83g:20032)

[21] J. Humphreys, Reflection Groups and Coxeter Groups. Cambridge Studies in Advanced Mathematics 29, Cambridge Univ. Press, 1990. MR.1066460 (92h:20002)

[22] P. Jedlička, A Combinatorial Construction of the Weak Order of a Coxeter Group. Comm. in Alg. 33 (2005), 1447-1460. MR2149070 (2006c:05143)

[23] V. Kac, Infinite-dimensional Lie algebras. Third edition. Cambridge University Press, Cambridge, 1990. MR1104219 (92k:17038)

[24] V. Kac, Infinite root systems, representations of graphs and invariant theory. Invent. Math. 56 (1980), no. 1, 57-92. MR.557581 (82j:16050)

[25] B. Keller, Cluster algebras, quiver representations and triangulated categories. arXiv: 0807.1960

[26] Y. Palu, Cluster characters for triangulated 2-Calabi-Yau categories. math/0703540

[27] A. Pilkington, Convex geometries on root systems. Comm. Algebra 34 (2006), no. 9, 31833202. MR2252665 (2007c:52001)

[28] N. Reading, Lattice and order properties of the poset of regions in a hyperplane arrangement. Algebra Universalis, 50 (2003), 179-205. MR2037526 (2004m:06006)

[29] N. Reading, Lattice congruences of the weak order. Order 21 (2004) no. 4, 315-344. MR2209128 (2007a:20036)

[30] N. Reading, Lattice congruences, fans and Hopf algebras. J. Combin. Theory Ser. A 110 (2005) no. 2, 237-273. MR2142177 (2006b:20054)

[31] N. Reading, Cambrian lattices. Adv. Math. 205 (2006), no. 2, 313-353. MR 2258260 (2007g:05195)

[32] N. Reading, Clusters, Coxeter-sortable elements and noncrossing partitions. Trans. Amer. Math. Soc. 359 (2007), no. 12, 5931-5958. MR2336311 (2009d:20093)

[33] N. Reading, Sortable elements and Cambrian lattices. Algebra Universalis 56 (2007), no. 3-4, 411-437. MR2318219 (2008d:20073)

[34] N. Reading and D. Speyer, Cambrian Fans, J. Eur. Math. Soc. (JEMS) 11 (2009), no. 2, 407-447. MR2486939 
[35] D. Speyer, Powers of Coxeter elements in infinite groups are reduced, Proc. Amer. Math. Soc. 137 (2009), no. 4, 1295-1302. MR2465651 (2009i:20079)

[36] S. Yang and A. Zelevinsky, Cluster algebras of finite type via Coxeter elements and principal minors, arXiv:0804.3303 Transformation Groups, to appear.

[37] G. Ziegler, Lectures on polytopes. Graduate Texts in Mathematics 152, Springer-Verlag, New York, 1995. MR1311028(96a:52011)

Department of Mathematics, North Carolina State University, Raleigh, North CarOLINA 27695

E-mail address: nathan_reading@ncsu.edu

Department of Mathematics, Massachusetts institute of Technology, Cambridge, MASSACHUSETTS 02139

E-mail address: speyer@math.mit.edu

Current address: Department of Mathematics, University of Michigan, Ann Arbor, Michigan 48109 